\title{
Search for squarks and gluinos with the ATLAS detector in final states with jets and missing transverse momentum using $\sqrt{s}=8 \mathrm{TeV}$ proton-proton collision data
}

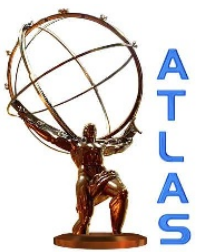

\section{The ATLAS collaboration}

\section{E-mail: atlas.publications@cern.ch}

ABSTRACT: A search for squarks and gluinos in final states containing high- $p_{\mathrm{T}}$ jets, missing transverse momentum and no electrons or muons is presented. The data were recorded in 2012 by the ATLAS experiment in $\sqrt{s}=8 \mathrm{TeV}$ proton-proton collisions at the Large Hadron Collider, with a total integrated luminosity of $20.3 \mathrm{fb}^{-1}$. Results are interpreted in a variety of simplified and specific supersymmetry-breaking models assuming that $R$-parity is conserved and that the lightest neutralino is the lightest supersymmetric particle. An exclusion limit at the $95 \%$ confidence level on the mass of the gluino is set at $1330 \mathrm{GeV}$ for a simplified model incorporating only a gluino and the lightest neutralino. For a simplified model involving the strong production of first- and second-generation squarks, squark masses below $850 \mathrm{GeV}(440 \mathrm{GeV})$ are excluded for a massless lightest neutralino, assuming mass degenerate (single light-flavour) squarks. In mSUGRA/CMSSM models with $\tan \beta=30, A_{0}=-2 m_{0}$ and $\mu>0$, squarks and gluinos of equal mass are excluded for masses below $1700 \mathrm{GeV}$. Additional limits are set for non-universal Higgs mass models with gaugino mediation and for simplified models involving the pair production of gluinos, each decaying to a top squark and a top quark, with the top squark decaying to a charm quark and a neutralino. These limits extend the region of supersymmetric parameter space excluded by previous searches with the ATLAS detector.

KeYwords: Hadron-Hadron Scattering, Supersymmetry

ARXIV EPRINT: 1405.7875 


\section{Contents}

1 Introduction 1

2 The ATLAS detector 2

3 Dataset and trigger 2

4 Monte Carlo data samples $\quad 3$

5 Event reconstruction $\quad 5$

6 Event selection $\quad 6$

$\begin{array}{lll}6.1 & \text { Signal regions } & 7\end{array}$

6.2 Control regions 9

$\begin{array}{lll}6.3 & \text { Validation regions } & 10\end{array}$

$\begin{array}{llr}7 & \text { Background estimation } & 11\end{array}$

$\begin{array}{lll}7.1 & \text { Overview } & 11\end{array}$

$\begin{array}{lll}7.2 & \text { Systematic uncertainties } & 14\end{array}$

$\begin{array}{lll}7.3 \text { Validation } & 16\end{array}$

$\begin{array}{llr}8 & \text { Results } & 16\end{array}$

$\begin{array}{llr}9 & \text { Interpretation } & 17\end{array}$

$\begin{array}{lr}10 \text { Conclusions } & 28\end{array}$

The ATLAS collaboration $\quad 36$

\section{Introduction}

Many extensions of the Standard Model (SM) include heavy coloured particles, some of which could be accessible at the Large Hadron Collider (LHC) [1]. The squarks $(\tilde{q})$ and gluinos $(\tilde{g})$ of supersymmetric (SUSY) theories [2-10] form one class of such particles. In these theories the squarks $\tilde{q}_{\mathrm{L}}$ and $\tilde{q}_{\mathrm{R}}$ are the partners of the left- and right-handed SM quarks respectively, while the gluinos $(\tilde{g})$ are the partners of the SM gluons. The partners of the neutral and charged SM gauge and Higgs bosons are respectively the neutralinos $\left(\tilde{\chi}^{0}\right)$ and charginos $\left(\tilde{\chi}^{ \pm}\right)$. This paper presents a search for these particles in final states containing only jets and large missing transverse momentum. Interest in this final state is motivated by the large number of $R$-parity-conserving [11-15] models in which squarks (including anti-squarks) and gluinos can be produced in pairs $(\tilde{g} \tilde{g}, \tilde{q} \tilde{q}, \tilde{q} \tilde{g})$ and can decay 
through $\tilde{q} \rightarrow q \tilde{\chi}_{1}^{0}$ and $\tilde{g} \rightarrow q \bar{q} \tilde{\chi}_{1}^{0}$ to weakly interacting lightest neutralinos, $\tilde{\chi}_{1}^{0}$. The $\tilde{\chi}_{1}^{0}$ is the lightest SUSY particle (LSP) in these models and escapes the detector unseen. Additional decay modes can include the production of charginos via $\tilde{q} \rightarrow q \tilde{\chi}^{ \pm}$(where $\tilde{q}$ and $q$ are of different flavour) and $\tilde{g} \rightarrow q \bar{q} \tilde{\chi}^{ \pm}$. Subsequent decay of these charginos to $W^{ \pm} \tilde{\chi}_{1}^{0}$ can lead to final states with still larger multiplicities of jets. The analysis presented here updates previous ATLAS results obtained using similar selections [16-18]. Further results of relevance to these models were published by the CMS collaboration [19-22].

In this analysis, events with reconstructed electrons or muons are vetoed to avoid overlap with a related ATLAS search [23]. The search strategy is optimised in the $\left(m_{\tilde{g}}, m_{\tilde{q}}\right)$ plane (where $m_{\tilde{g}}, m_{\tilde{q}}$ are the gluino and squark masses respectively) for a range of models, including simplified models in which all other supersymmetric particles, except for the lightest neutralino, are assigned masses beyond the reach of the LHC. Although interpreted in terms of SUSY models, the main results of this analysis (the data and expected background event counts after selection requirements) are relevant for constraining any model of new physics that predicts production of jets in association with missing transverse momentum.

\section{The ATLAS detector}

The ATLAS detector [24] is a multipurpose particle physics detector with a forwardbackward symmetric cylindrical geometry and nearly $4 \pi$ coverage in solid angle. ${ }^{1}$ The detector features four superconducting magnet systems, which comprise a thin solenoid surrounding inner tracking detectors (covering $|\eta|<2.5$ ) and, outside a calorimeter system, three large toroids supporting a muon spectrometer (covering $|\eta|<2.7$, with trigger coverage in the region $|\eta|<2.4$ ). The calorimeters are of particular importance to this analysis. In the pseudorapidity region $|\eta|<3.2$, high-granularity liquid-argon (LAr) electromagnetic (EM) sampling calorimeters are used. An iron/scintillator-tile calorimeter provides hadronic coverage over $|\eta|<1.7$. The end-cap and forward regions, spanning $1.5<|\eta|<4.9$, are instrumented with LAr calorimeters for both EM and hadronic energy measurements.

\section{Dataset and trigger}

The dataset used in this analysis was collected in 2012 with the LHC operating at a centre-of-mass energy of $8 \mathrm{TeV}$. Application of beam, detector and data-quality requirements resulted in a total integrated luminosity of $20.3 \mathrm{fb}^{-1}$. The uncertainty on the integrated luminosity is $\pm 2.8 \%$, derived by following the same methodology as that detailed in ref. [25]. During the data-taking period, the peak instantaneous luminosity per LHC fill was typically $7 \times 10^{33} \mathrm{~cm}^{-2} \mathrm{~s}^{-1}$, while the mean number of proton-proton interactions per LHC bunch crossing was 21. The trigger required events to contain a jet with an uncorrected

\footnotetext{
${ }^{1}$ ATLAS uses a right-handed coordinate system with its origin at the nominal interaction point in the centre of the detector and the $z$-axis along the beam pipe. Cylindrical coordinates $(r, \phi)$ are used in the transverse plane, $\phi$ being the azimuthal angle around the beam pipe. The pseudorapidity $\eta$ is defined in terms of the polar angle $\theta$ by $\eta=-\ln \tan (\theta / 2)$.
} 
transverse momentum $\left(p_{\mathrm{T}}\right)$ above $80 \mathrm{GeV}$ and an uncorrected missing transverse momentum above $100 \mathrm{GeV}$. The trigger reached its full efficiency for events with a reconstructed jet with $p_{\mathrm{T}}$ exceeding $130 \mathrm{GeV}$ and more than $160 \mathrm{GeV}$ of missing transverse momentum, which are requirements of the event selections considered in this analysis. Auxiliary data samples used to estimate the yields of background events in the analysis were selected using triggers requiring a single isolated electron $\left(p_{\mathrm{T}}>24 \mathrm{GeV}\right)$, muon $\left(p_{\mathrm{T}}>24 \mathrm{GeV}\right)$ or photon $\left(p_{\mathrm{T}}>120 \mathrm{GeV}\right)$.

\section{Monte Carlo data samples}

Monte Carlo (MC) data samples are used to develop the analysis, optimise the selections, estimate backgrounds and assess sensitivity to specific SUSY signal models. The SM background processes considered are those which can lead to events with jets and missing transverse momentum. The processes considered together with the MC generators, crosssection calculations and parton distribution functions (PDFs) used are listed in table 1. The $\gamma+$ jets MC data samples are used to estimate the $Z+$ jets background through a datadriven normalisation procedure described in section 7 . When considering the dominant $W / Z / \gamma^{*}+$ jets and $t \bar{t}$ background processes, two generators are used in each case, with results from the second being used to evaluate systematic uncertainties in background estimates obtained with the first. When using the baseline POWHEG-BOX+PYTHIA top quark pair production sample, events are reweighted in bins of $p_{\mathrm{T}}(t \bar{t})$ to match the top quark pair differential cross-section observed in ATLAS data [26, 27]. No corrections are applied to the alternative MC@NLO sample used for systematic uncertainty evaluation, which reproduces more accurately the $p_{\mathrm{T}}(t \bar{t})$ distribution measured in data. MC@NLO is nevertheless not used as the default generator for this process as it is observed to reproduce less accurately high jet-multiplicity events.

SUSY signal samples are generated with HERWIG++-2.5.2 [56] or MADGRAPH5.0 matched to PYTHIA-6.426, using PDF set CTEQ6L1. The specific generators used for each model are discussed in section 9. The MADGRAPH samples are produced using the AUET2B tune (also used for some background samples — see table 1). The MLM matching scheme [57] is used with up to one additional jet in the MADGRAPH matrix element, and a MADGRAPH $k_{\mathrm{t}}$ measure cut-off and a PYTHIA jet measure cut-off both set to 0.25 times the mass scale of the SUSY particles produced in the hard process, with a maximum value of $500 \mathrm{GeV}$. Signal cross-sections are calculated to next-to-leading order in the strong coupling constant, including the resummation of soft gluon emission at next-to-leading-logarithmic accuracy (NLO+NLL) [58-62]. In each case the nominal crosssection and its uncertainty are taken from an ensemble of cross-section predictions using different PDF sets and factorisation and renormalisation scales, as described in ref. [63]. For the mSUGRA/CMSSM [64-69] and non-universal Higgs mass model with gaugino mediation (NUHMG) [70] samples the SUSY particle mass spectra and decay tables are calculated with SUSY-HIT [71] interfaced to the SOFTSUSY spectrum generator [72] and SDECAY [73]. 


\begin{tabular}{|c|c|c|c|c|}
\hline Process & $\begin{array}{l}\text { Generator } \\
+ \text { frag./had. }\end{array}$ & $\begin{array}{l}\text { Cross-section } \\
\text { order in } \alpha_{s}\end{array}$ & Tune & PDF set \\
\hline$W+$ jets & SHERPA-1.4.0 [28] & NNLO [29] & SHERPA default & CT10 [30] \\
\hline$W+$ jets $(\bullet)$ & $\begin{array}{c}\text { ALPGEN-2.14 [31] } \\
+ \text { HERWIG-6.520 }[34,35]\end{array}$ & NNLO [29] & AUET2B [32] & CTEQ6L1 [33] \\
\hline$Z / \gamma^{*}+$ jets & SHERPA-1.4.0 & NNLO [29] & SHERPA default & CT10 \\
\hline$Z / \gamma^{*}+$ jets $(\bullet)$ & $\begin{aligned} & \text { ALPGEN-2.14 } \\
+ & \text { HERWIG-6.520 }\end{aligned}$ & NNLO [29] & AUET2B & CTEQ6L1 \\
\hline$\gamma+$ jets & SHERPA-1.4.0 & $\mathrm{LO}$ & SHERPA default & CT10 \\
\hline$\gamma+$ jets $(\bullet)$ & $\begin{aligned} & \text { ALPGEN-2.14 } \\
+ & \text { HERWIG-6.520 }\end{aligned}$ & $\mathrm{LO}$ & AUET2B & CTEQ6L1 \\
\hline$t \bar{t}$ & $\begin{array}{l}\text { POWHEG-BOX-1.0 [36-38] } \\
\quad+\text { PYTHIA-6.426 [41] }\end{array}$ & NNLO+NNLL $[39,40]$ & $\begin{array}{c}\text { Perugia } 2011 \mathrm{C} \\
{[42,43]}\end{array}$ & CT10 \\
\hline$t \bar{t}(\bullet)$ & $\begin{array}{c}\text { MC@NLO-4.03 }[44,45] \\
+ \text { HERWIG-6.520 }\end{array}$ & NNLO+NNLL $[39,40]$ & AUET2B & CT10 \\
\hline \multicolumn{5}{|l|}{ Single top } \\
\hline$t$-channel & $\begin{array}{l}\text { ACERMC-38 [46] } \\
+ \text { PYTHIA-6.426 }\end{array}$ & NNLO+NNLL [47] & AUET2B & CTEQ6L1 \\
\hline$s$-channel, $W t$ & $\begin{array}{c}\text { MC@NLO-4.03 } \\
+ \text { HERWIG-6.520 }\end{array}$ & NNLO+NNLL $[48,49]$ & AUET2B & CT10 \\
\hline$t \bar{t}+\mathrm{EW}$ boson & $\begin{array}{l}\text { MADGRAPH-5.0 [50] } \\
\text { + PYTHIA-6.426 }\end{array}$ & NLO [51-53] & AUET2B & CTEQ6L1 \\
\hline $\begin{array}{c}\text { Dibosons } \\
W W, W Z, Z Z, \\
W \gamma \text { and } Z \gamma\end{array}$ & SHERPA-1.4.0 & NLO $[54,55]$ & SHERPA default & CT10 \\
\hline
\end{tabular}

Table 1. The Standard Model background Monte Carlo simulation samples used in this article. The generators, the order in $\alpha_{\mathrm{s}}$ of cross-section calculations used for yield normalisation (leading order/LO, next-to-leading order/NLO, next-to-next-to-leading order/NNLO, next-to-next-to-leading logarithm/NNLL), tunes used for the underlying event and PDF sets are shown. Samples denoted with $(\bullet)$ are used for evaluation of systematic uncertainties. For the $\gamma+$ jets process the LO cross-section is taken directly from the MC generator.

The MC samples are generated using the same parameter set as in refs. [74-76]. SM background samples are passed through either the full ATLAS detector simulation [77] based on GEANT4 [78], or, when larger samples are required, through a fast simulation using a parameterisation of the performance of the ATLAS EM and hadronic calorimeters [79] and GEANT4 elsewhere $\left(W / Z / \gamma+\right.$ jets samples with boson $p_{\mathrm{T}}<280 \mathrm{GeV}$ and POWHEG-BOX+PYTHIA $t \bar{t}$ samples only). All SUSY signal samples with the exception of mSUGRA/CMSSM model samples (which are produced with the GEANT4 simulation) are passed through the fast simulation. The fast simulation of SUSY signal events was validated against full GEANT4 simulation for several signal model points. Differing pile-up (multiple proton-proton interactions in the same or neighbouring bunchcrossings) conditions as a function of the instantaneous luminosity are taken into account by overlaying simulated minimum-bias events generated with PYTHIA-8 onto the hardscattering process and reweighting them according to the distribution of the mean number of interactions observed in data. 


\section{Event reconstruction}

Jet candidates are reconstructed using the anti- $k_{\mathrm{T}}$ jet clustering algorithm $[80,81]$ with a radius parameter of 0.4 . The inputs to this algorithm are the energies of clusters $[82,83]$ of calorimeter cells seeded by those with energy significantly above the measured noise. Jet momenta are constructed by performing a four-vector sum over these cell clusters, treating each as an $(E, \vec{p})$ four-vector with zero mass. The jets are corrected for energy from pile-up using a method, suggested in ref. [84], which estimates the pile-up activity in any given event as well as the sensitivity of any given jet to pile-up. The method subtracts a contribution from the jet energy equal to the product of the jet area and the average energy density of the event [85]. The local cluster weighting (LCW) jet calibration method [82, 86] is used to classify topological cell clusters within the jets as being of either electromagnetic or hadronic origin, and based on this classification applies specific energy corrections derived from a combination of MC simulation and data. Further corrections, referred to as 'jet energy scale' or 'JES' corrections below, are derived from MC simulation and data and used to calibrate the energies of jets to the scale of their constituent particles [82, 87]. Only jet candidates with $p_{\mathrm{T}}>20 \mathrm{GeV}$ and $|\eta|<4.5$ after all corrections are retained. Jets are identified as originating from heavy-flavour ( $b$ and $c$ quark) decays using the 'MV1' neural-network-based $b$-tagging algorithm, with an operating point with an efficiency of $70 \%$ and a light quark rejection factor of 140 determined with simulated $t \bar{t}$ events [88]. Candidate $b$-tagged jets must possess $p_{\mathrm{T}}>40 \mathrm{GeV}$ and $|\eta|<2.5$.

Two different classes of reconstructed leptons (electrons or muons) are used in this analysis. When selecting samples of potential SUSY signal events, events containing any 'baseline' electrons or muons are rejected, as described in section 6.1. The selections applied to baseline leptons are designed to maximise the efficiency with which $W+$ jet and top quark background events are rejected. When selecting 'control region' samples for the purpose of estimating residual $W$ +jets and top quark backgrounds, as described in section 6.2, additional requirements are applied to improve the purity of the samples. These leptons will be referred to as 'high-purity' leptons and form a subset of the baseline leptons.

Baseline electron candidates are required to have $p_{\mathrm{T}}>10 \mathrm{GeV}$ and $|\eta|<2.47$, and to satisfy 'medium' electron shower shape and track selection criteria based upon those described in ref. [89], but modified to reduce the impact of pile-up and to match tightened trigger requirements in 2012 data. High-purity electron candidates additionally must have $p_{\mathrm{T}}>25 \mathrm{GeV}$, must satisfy tighter selection criteria, must have transverse and longitudinal impact parameters within $1.0 \mathrm{~mm}$ and $2.0 \mathrm{~mm}$, respectively, of the primary vertex, which is defined to be the reconstructed vertex with the highest $\sum p_{\mathrm{T}}^{2}$ of tracks, and must be isolated. ${ }^{2}$ Baseline muon candidates are formed by combining information from the muon spectrometer and inner tracking detectors as described in ref. [90] and are required to have $p_{\mathrm{T}}>10 \mathrm{GeV}$ and $|\eta|<2.4$. High-purity muon candidates must additionally have

\footnotetext{
${ }^{2}$ The scalar sum of the transverse momenta of tracks, other than that from the electron itself, within a cone of $\Delta R \equiv \sqrt{(\Delta \eta)^{2}+(\Delta \phi)^{2}}=0.2$ around the electron must be less than $10 \%$ of the $p_{\mathrm{T}}$ of the electron.
} 
$p_{\mathrm{T}}>25 \mathrm{GeV},|\eta|<2.4$, transverse and longitudinal impact parameters within $0.2 \mathrm{~mm}$ and $1.0 \mathrm{~mm}$, respectively, of the primary vertex and must be isolated. ${ }^{3}$

After the selections described above, ambiguities between candidate jets with $|\eta|<2.8$ and leptons are resolved as follows. First, any such jet candidate lying within a distance $\Delta R \equiv \sqrt{(\Delta \eta)^{2}+(\Delta \phi)^{2}}=0.2$ of a baseline electron is discarded; then any lepton candidate (baseline or high-purity) remaining within a distance $\Delta R=0.4$ of any surviving jet candidate is discarded.

The measurement of the missing transverse momentum two-dimensional vector $\mathbf{E}_{\mathrm{T}}^{\text {miss }}$ (and its magnitude $E_{\mathrm{T}}^{\mathrm{miss}}$ ) is based on the calibrated transverse momenta of all jet and baseline lepton candidates and all calorimeter energy clusters not associated with such objects [91, 92]. Following the calculation of the value of $\mathbf{E}_{\mathrm{T}}^{\mathrm{miss}}$, all jet candidates with $|\eta|>2.8$ are discarded. Thereafter, the remaining baseline lepton and jet candidates are considered "reconstructed", and the term "candidate" is dropped. In the MC simulation, reconstructed baseline or high-purity lepton and $b$-tagged jet identification efficiencies and misidentification probabilities are corrected using factors derived from data control regions.

Reconstructed photons are used to constrain $Z+$ jet backgrounds (see section 6.2), although they are not used in the main signal event selection. Photon candidates are required to possess $p_{\mathrm{T}}>130 \mathrm{GeV}$ and $|\eta|<1.37$ or $1.52<|\eta|<2.47$, to satisfy photon shower shape and electron rejection criteria [93], and to be isolated. ${ }^{4}$ Ambiguities between candidate jets and photons (when used in the event selection) are resolved by discarding any jet candidates lying within $\Delta R=0.2$ of a photon candidate. The transverse momenta of the resulting reconstructed photons are taken into account when calculating $\mathbf{E}_{\mathrm{T}}^{\mathrm{miss}}$.

Reconstructed $\tau$-leptons are not used in this analysis when selecting potential signal events or control region data samples; however, they are used to validate some of the estimates of $W+$ jets and top quark backgrounds, as described in section 6.3. The $\tau$-leptons are reconstructed using a $p_{\mathrm{T}}$-correlated track counting algorithm described in ref. [94]. The purity of the validation event samples selecting background events containing hadronically decaying $\tau$-leptons ranges from $65 \%$ to $90 \%$.

\section{Event selection}

Events selected by the trigger are discarded if they contain any candidate jets failing to satisfy quality selection criteria designed to suppress detector noise and non-collision backgrounds, or if they lack a reconstructed primary vertex associated with five or more tracks $[95,96]$. The criteria applied to candidate jets include requirements on the fraction of the transverse momentum of the jet carried by reconstructed charged particle tracks, and on the fraction of the jet energy contained in the EM layers of the calorimeter. A consequence of these requirements is that events containing hard isolated photons have a high probability of failing to satisfy the signal event selection criteria, under which ambiguities between candidate jets and photons are not resolved (see section 5).

\footnotetext{
${ }^{3}$ The scalar sum of the transverse momenta of tracks, other than that from the muon itself, within a cone of $\Delta R=0.2$ around the muon must be less than $1.8 \mathrm{GeV}$.

${ }^{4}$ The transverse energy in the calorimeter, other than from that from the photon itself and corrected for noise and pile-up, within a cone of $\Delta R=0.4$ around the photon must be less than $4 \mathrm{GeV}$.
} 
This analysis aims to search for the production of heavy SUSY particles decaying into jets and stable lightest neutralinos, with the latter creating missing transverse momentum. Because of the high mass scale expected for the SUSY signal, the 'effective mass', $m_{\mathrm{eff}}$, is a powerful discriminant between the signal and most SM backgrounds. When selecting events with at least $N_{\mathrm{j}}$ jets, $m_{\mathrm{eff}}\left(N_{\mathrm{j}}\right)$ is defined to be the scalar sum of the transverse momenta of the leading $N_{\mathrm{j}}$ jets and $E_{\mathrm{T}}^{\mathrm{miss}}$. The final signal selection uses requirements on $m_{\mathrm{eff}}$ (incl.), which sums over all jets with $p_{\mathrm{T}}>40 \mathrm{GeV}$ and $E_{\mathrm{T}}^{\text {miss }}$. Requirements placed on $m_{\text {eff }}$ and $E_{\mathrm{T}}^{\text {miss }}$, which suppress the multi-jet background in which jet energy mismeasurement generates missing transverse momentum, formed the basis of the previous ATLAS jets + $E_{\mathrm{T}}^{\text {miss }}+0$-lepton SUSY searches [16-18]. The same strategy is adopted in this analysis, and is described below.

\subsection{Signal regions}

In order to achieve maximal reach over the $\left(m_{\tilde{g}}, m_{\tilde{q}}\right)$-plane, a variety of signal regions (SRs) are defined. Squarks typically generate at least one jet in their decays, for instance through $\tilde{q} \rightarrow q \tilde{\chi}_{1}^{0}$, while gluinos typically generate at least two jets, for instance through $\tilde{g} \rightarrow q \bar{q} \tilde{\chi}_{1}^{0}$. Processes contributing to $\tilde{q} \tilde{q}, \tilde{q} \tilde{g}$ and $\tilde{g} \tilde{g}$ final states therefore lead to events containing at least two, three or four jets, respectively. Decays of heavy SUSY and SM particles produced in longer $\tilde{q}$ and $\tilde{g}$ cascade decays (e.g. $\tilde{\chi}_{1}^{ \pm} \rightarrow q q^{\prime} \tilde{\chi}_{1}^{0}$ ) tend to further increase the jet multiplicity in the final-state.

Fifteen inclusive SRs characterised by increasing minimum jet-multiplicity from two to six, are defined in table 2. In all cases, events are discarded if they contain baseline electrons or muons with $p_{\mathrm{T}}>10 \mathrm{GeV}$. Several SRs may be defined for the same jetmultiplicity, distinguished by increasing background rejection, ranging from 'very loose' (labelled 'l-') to 'very tight' (labelled ' $t+$ '). The lower jet-multiplicity SRs focus on models characterised by squark pair production with short decay chains, while those requiring high jet-multiplicity are optimised for gluino pair production and/or long cascade decay chains.

Requirements are placed upon $\Delta \phi\left(\text { jet, } \mathbf{E}_{\mathrm{T}}^{\text {miss }}\right)_{\min }$, which is defined to be the smallest of the azimuthal separations between $\mathbf{E}_{\mathrm{T}}^{\mathrm{miss}}$ and the reconstructed jets. For the 2-jet and 3-jet SRs the selection requires $\Delta \phi\left(\text { jet, } \mathbf{E}_{\mathrm{T}}^{\mathrm{miss}}\right)_{\min }>0.4$ using up to three leading jets with $p_{\mathrm{T}}>40 \mathrm{GeV}$ if present in the event. For the other SRs an additional requirement $\Delta \phi\left(\text { jet, } \mathbf{E}_{\mathrm{T}}^{\text {miss }}\right)_{\min }>0.2$ is placed on all jets with $p_{\mathrm{T}}>40 \mathrm{GeV}$. Requirements on $\Delta \phi\left(\text { jet }, \mathbf{E}_{\mathrm{T}}^{\mathrm{miss}}\right)_{\min }$ and $E_{\mathrm{T}}^{\mathrm{miss}} / m_{\mathrm{eff}}\left(N_{\mathrm{j}}\right)$ are designed to reduce the background from multijet processes.

In the SRs $2 \mathrm{jl}, 2 \mathrm{jm}, 2 \mathrm{jt}, 4 \mathrm{jl}$ and $4 \mathrm{jl}$ - the requirement on $E_{\mathrm{T}}^{\mathrm{miss}} / m_{\mathrm{eff}}\left(N_{\mathrm{j}}\right)$ is replaced by a requirement on $E_{\mathrm{T}}^{\text {miss }} / \sqrt{H_{\mathrm{T}}}$ (where $H_{\mathrm{T}}$ is defined as the scalar sum of the transverse momenta of all $p_{\mathrm{T}}>40 \mathrm{GeV}$ jets), which was found to lead to enhanced sensitivity to models characterised by $\tilde{q} \tilde{q}$ production. Two of the $\mathrm{SRs}(2 \mathrm{jW}$ and $4 \mathrm{jW})$ place additional requirements on the invariant masses $m\left(W_{\text {cand }}\right)$ of candidate $W$ bosons decaying to hadrons, by requiring $60 \mathrm{GeV}<m\left(W_{\text {cand }}\right)<100 \mathrm{GeV}$. Candidate $W$ bosons are reconstructed from single high-mass jets (unresolved candidates - ' $W \rightarrow j$ ' in table 2) or from pairs of jets (resolved candidates — ' $W \rightarrow j j$ ' in table 2). Resolved candidates are reconstructed using an iterative procedure which assigns each jet to a unique pair with minimum separation 


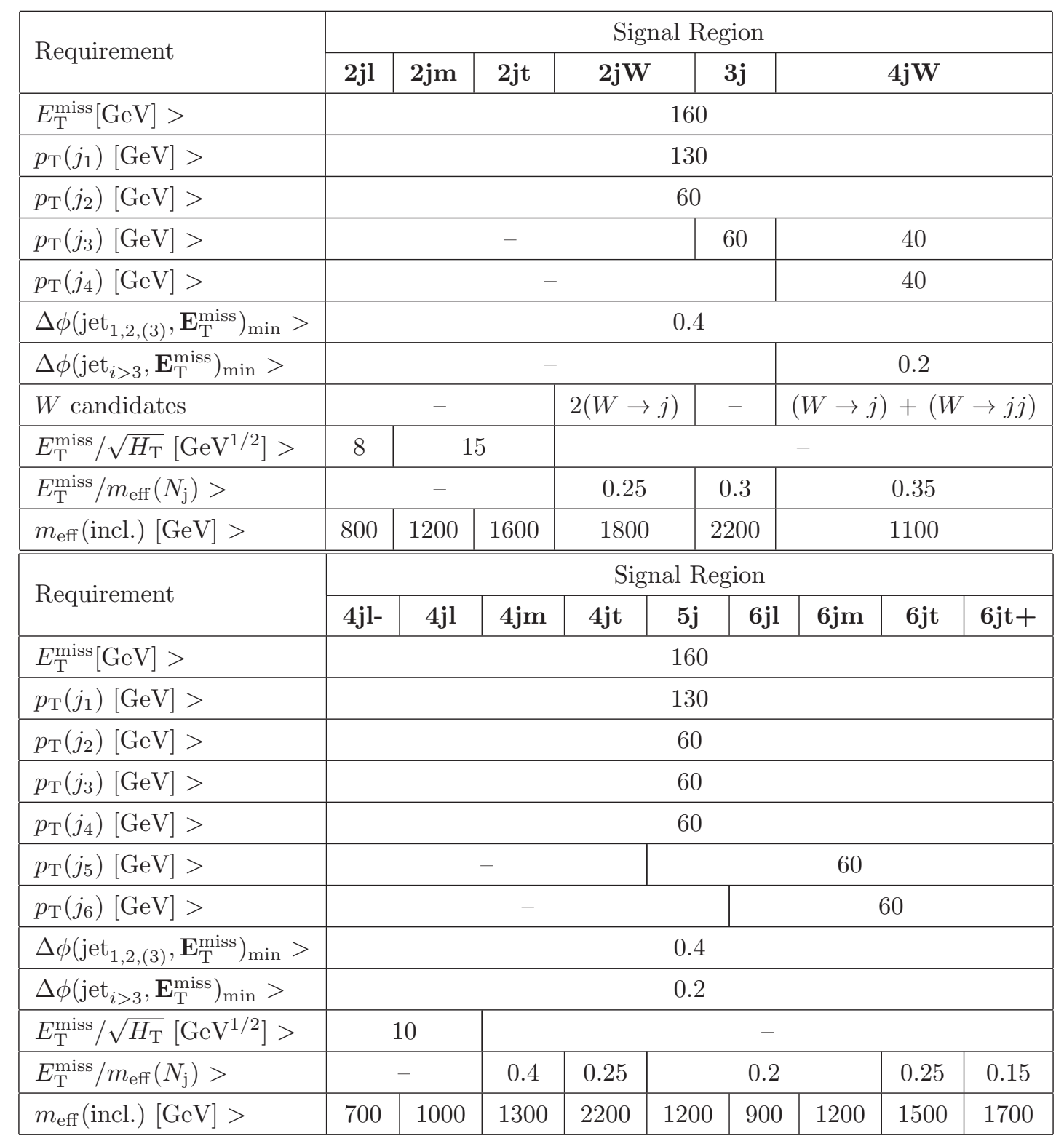

Table 2. Selection criteria used to define each of the signal regions in the analysis. Each SR is labelled with the inclusive jet-multiplicity considered (' $2 \mathrm{j}$ ', ' $3 \mathrm{j}$ ' etc.) together with the degree of background rejection. The latter is denoted by labels 'l-' ('very loose'), 'l' ('loose'), 'm' ('medium'), 't' ('tight') and 't+' ('very tight'). The $E_{\mathrm{T}}^{\text {miss }} / m_{\text {eff }}\left(N_{\mathrm{j}}\right)$ cut in any $N_{\mathrm{j}}$-jet channel uses a value of $m_{\text {eff }}$ constructed from only the leading $N_{\mathrm{j}}$ jets $\left(m_{\mathrm{eff}}\left(N_{\mathrm{j}}\right)\right)$. However, the final $m_{\text {eff }}$ (incl.) selection, which is used to define the signal regions, includes all jets with $p_{\mathrm{T}}>40 \mathrm{GeV}$. In SR $2 \mathrm{jW}$ and SR $4 \mathrm{jW}$ a requirement $60 \mathrm{GeV}<m\left(W_{\text {cand }}\right)<100 \mathrm{GeV}$ is placed on the masses of candidate resolved or unresolved hadronically decaying $W$ bosons, as described in the text.

$\Delta R(j, j)$. SR $2 \mathrm{jW}$ requires two unresolved candidates, while SR $4 \mathrm{jW}$ requires one resolved candidate and one unresolved candidate. These SRs are designed to improve sensitivity to models predicting enhanced branching ratios for cascade $\tilde{q}$ or $\tilde{g}$ decay via $\tilde{\chi}_{1}^{ \pm}$to $W$ and $\tilde{\chi}_{1}^{0}$, in cases where the $\tilde{\chi}_{1}^{ \pm}$is nearly degenerate in mass with the $\tilde{q}$ or $\tilde{g}$ (see section 9 ). 
Standard Model background processes contribute to the event counts in the signal regions. The dominant sources are: $Z+$ jets, $W+$ jets, top quark pairs, single top quarks, and multiple jets. The production of boson $(W / Z / \gamma)$ pairs in which at least one boson decays to charged leptons and/or neutrinos (referred to as 'dibosons' below) is a small component (in most SRs $\lesssim 10 \%$, up to $\sim 30 \%$ in SR 6jt, predominantly $W Z$ ) of the total background and is estimated with MC simulated data normalised to NLO cross-section predictions. The majority of the $W+$ jets background is composed of $W \rightarrow \tau \nu$ events in which the $\tau$ lepton decays to hadrons, with additional contributions from $W \rightarrow e \nu, \mu \nu$ events in which no baseline electron or muon is reconstructed. The largest part of the $Z+$ jets background comes from the irreducible component in which $Z \rightarrow \nu \bar{\nu}$ decays generate large $E_{\mathrm{T}}^{\text {miss }}$. Top quark pair production followed by semileptonic decays, in particular $t \bar{t} \rightarrow b \bar{b} \tau \nu q q^{\prime}$ with the $\tau$-lepton decaying to hadrons, as well as single top quark events, can also generate large $E_{\mathrm{T}}^{\mathrm{miss}}$ and satisfy the jet and lepton-veto requirements at a non-negligible rate. The multijet background in the signal regions is caused by mis-reconstruction of jet energies in the calorimeters generating missing transverse momentum, as well as by neutrino production in semileptonic decays of heavy-flavour quarks.

\subsection{Control regions}

To estimate the backgrounds in a consistent and robust fashion, four control regions (CRs) are defined for each of the 15 signal regions, giving $60 \mathrm{CRs}$ in total. The orthogonal CR event selections are designed to provide independent data samples enriched in particular background sources. The CR selections are optimised to maintain adequate statistical weight and negligible SUSY signal contamination, while minimising as far as possible the systematic uncertainties arising from the extrapolation of the CR event yield to the expectation in the SR. This latter requirement is addressed through the use wherever possible of CR $m_{\text {eff }}$ (incl.) selections which match those used in the SR.

The $\mathrm{CR}$ definitions are listed in table 3. The $\mathrm{CR} \gamma$ control region is used to estimate the contribution of $Z(\rightarrow \nu \nu)+$ jets background events to each SR by selecting a sample of $\gamma+$ jets events with $p_{\mathrm{T}}(\gamma)>130 \mathrm{GeV}$ and then treating the reconstructed photon as contributing to $E_{\mathrm{T}}^{\text {miss }}$. For $p_{\mathrm{T}}(\gamma)$ greater than $m_{Z}$ the kinematics of such events strongly resemble those of $Z$ +jets events [16]. CRQ uses reversed selection requirements placed on $\Delta \phi\left(\text { jet, } \mathbf{E}_{\mathrm{T}}^{\text {miss }}\right)_{\text {min }}$ and on $E_{\mathrm{T}}^{\text {miss }} / m_{\mathrm{eff}}\left(N_{\mathrm{j}}\right)\left(E_{\mathrm{T}}^{\text {miss }} / \sqrt{H_{\mathrm{T}}}\right.$ where appropriate $)$ to produce data samples enriched in multi-jet background events. CRW and CRT use respectively a $b$-jet veto or $b$-jet requirement together with a requirement on the transverse mass $m_{\mathrm{T}}$ of a high-purity lepton with $p_{\mathrm{T}}>25 \mathrm{GeV}$ and $\mathbf{E}_{\mathrm{T}}^{\text {miss }}$ to select samples of $W(\rightarrow \ell \nu)+$ jets and semileptonic $t \bar{t}$ background events. These samples are used to estimate respectively the $W+$ jets and combined $t \bar{t}$ and single-top background populations, treating the lepton as a jet with the same momentum to model background events in which a hadronically decaying $\tau$-lepton is produced. With the exception of SR 2jl, the CRW and CRT selections do not use the SR selection requirements applied to $\Delta \phi\left(\text { jet, } \mathbf{E}_{\mathrm{T}}^{\mathrm{miss}}\right)_{\min }$ or $E_{\mathrm{T}}^{\mathrm{miss}} / m_{\mathrm{eff}}\left(N_{\mathrm{j}}\right)$ $\left(E_{\mathrm{T}}^{\text {miss }} / \sqrt{H_{\mathrm{T}}}\right.$ where appropriate) in order to increase CR data event statistics without significantly increasing theoretical uncertainties associated with the background estimation procedure. For the same reason, the final $m_{\mathrm{eff}}$ (incl.) requirements are loosened to $1300 \mathrm{GeV}$ 


\begin{tabular}{|c|c|c|c|}
\hline $\mathrm{CR}$ & SR background & $\mathrm{CR}$ process & CR selection \\
\hline $\mathrm{CR} \gamma$ & $Z(\rightarrow \nu \nu)+$ jets & $\gamma+$ jets & Isolated photon \\
\hline CRQ & Multi-jets & Multi-jets & $\begin{array}{l}\text { SR with reversed requirements on (i) } \Delta \phi\left(\text { jet, } \mathbf{E}_{\mathrm{T}}^{\text {miss }}\right)_{\text {min }} \\
\text { and (ii) } E_{\mathrm{T}}^{\text {miss }} / m_{\text {eff }}\left(N_{\mathrm{j}}\right) \text { or } E_{\mathrm{T}}^{\text {miss }} / \sqrt{H_{\mathrm{T}}}\end{array}$ \\
\hline CRW & $W(\rightarrow \ell \nu)+$ jets & $W(\rightarrow \ell \nu)+$ jets & $30 \mathrm{GeV}<m_{\mathrm{T}}\left(\ell, E_{\mathrm{T}}^{\text {miss }}\right)<100 \mathrm{GeV}, b$-veto \\
\hline CRT & $t \bar{t}$ and single- $t$ & $t \bar{t} \rightarrow b \bar{b} q q^{\prime} \ell \nu$ & $30 \mathrm{GeV}<m_{\mathrm{T}}\left(\ell, E_{\mathrm{T}}^{\text {miss }}\right)<100 \mathrm{GeV}, b$-tag \\
\hline
\end{tabular}

Table 3. Control regions used in the analysis. Also listed are the main targeted background in the $\mathrm{SR}$ in each case, the process used to model the background, and the main CR requirement(s) used to select this process. The transverse momenta of high-purity leptons (photons) used to select CR events must exceed 25 (130) GeV.

in CRW and CRT of SR 6jt. The purity of the control regions for the background process targeted in each case ranges from $48 \%$ to $97 \%$.

Example CR $m_{\text {eff }}$ (incl.) distributions before the final cut on this quantity for SRs $2 \mathrm{jl}, 2 \mathrm{jm}$ and $2 \mathrm{jt}$ are shown in figure 1 . Jet and dijet mass distributions (respectively for unresolved and resolved $W$ candidates) in CRW and CRT of SRs $2 \mathrm{jW}$ and $4 \mathrm{jW}$ are shown in figure 2. The MC $m_{\text {eff }}$ (incl.) distributions in figure 1 are somewhat harder than the data, with better agreement seen at low values of $m_{\mathrm{eff}}$ (incl.). This issue is seen also in the SR $m_{\text {eff }}$ (incl.) distributions (see section 8 ) and is ameliorated in the SR background estimates using a combined fit to the CR observations (see section 7.1). The discrepancy is most pronounced for CR $\gamma$ and CRW and may be related to the overestimation by SHERPA (and also ALPGEN) of the $Z$ boson differential cross-section at high $p_{\mathrm{T}}$ observed in ref. [97].

\subsection{Validation regions}

Cross-checks of the background estimates (see section 7.3) are performed using several 'validation region' (VR) samples selected with requirements, distinct from those used in the control regions, which maintain a low probability of signal contamination. CR $\gamma$ estimates of the $Z(\rightarrow \nu \bar{\nu})+$ jets background are validated with samples of $Z(\rightarrow \ell \ell)+$ jets events selected by requiring high-purity lepton pairs of opposite sign and identical flavour for which the dilepton invariant mass lies within $25 \mathrm{GeV}$ of the mass of the $Z$ boson (VRZ). In VRZ the leptons are treated as contributing to $E_{\mathrm{T}}^{\text {miss }}$. CRW and CRT estimates of the $W+$ jets and top quark background are validated with CRW and CRT events with the signal region $\Delta \phi\left(\text { jet, } \mathbf{E}_{\mathrm{T}}^{\text {miss }}\right)_{\min }$ and $E_{\mathrm{T}}^{\text {miss }} / m_{\mathrm{eff}}\left(N_{\mathrm{j}}\right)$ or $E_{\mathrm{T}}^{\text {miss }} / \sqrt{H_{\mathrm{T}}}$ (as appropriate) requirements reinstated, and with the lepton treated either as a jet (VRW, VRT) or as contributing to $E_{\mathrm{T}}^{\text {miss }}$ $(\mathrm{VRW} \nu, \mathrm{VRT} \nu)$. Further validation of CRW and CRT estimates is provided by validation regions in which at least one hadronically decaying $\tau$-lepton is reconstructed, without $(\mathrm{VRW} \tau)$ or with $(\mathrm{VRT} \tau)$ a requirement of a $b$-tagged jet. CRQ estimates of the multi-jet background are validated with validation regions for which the CRQ selection is applied with the signal region $E_{\mathrm{T}}^{\text {miss }} / m_{\mathrm{eff}}\left(N_{\mathrm{j}}\right)\left(E_{\mathrm{T}}^{\text {miss }} / \sqrt{H_{\mathrm{T}}}\right)$ requirement reinstated (VRQa), or with a requirement of an intermediate value of $\Delta \phi\left(\mathrm{jet}, \mathbf{E}_{\mathrm{T}}^{\mathrm{miss}}\right)_{\min }$ applied (VRQb). 

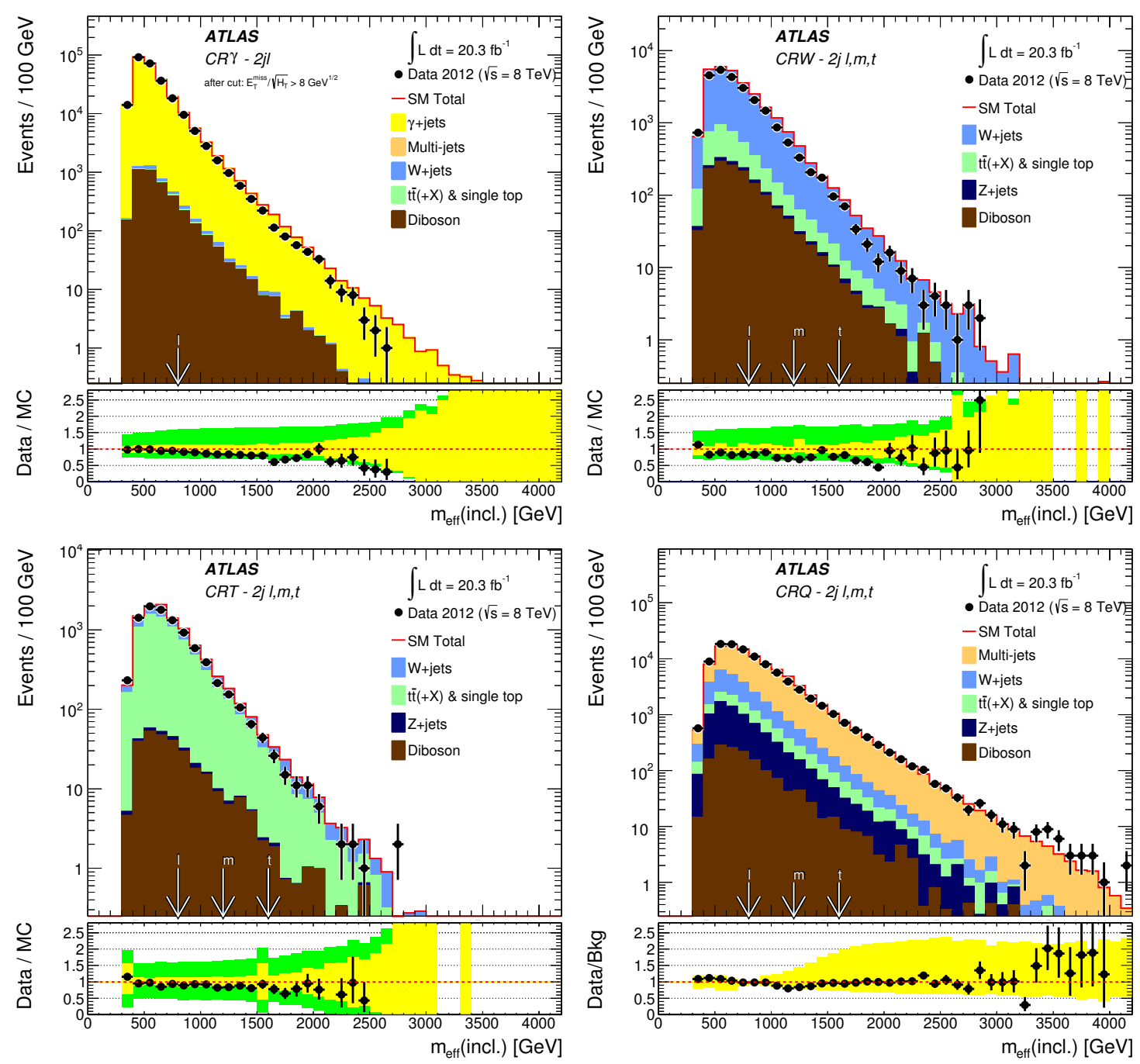

Figure 1. Observed $m_{\text {eff }}$ (incl.) distributions in control regions $\mathrm{CR} \gamma$ (top left, for SR 2jl selection criteria only), CRW (top right), CRT (bottom left) and CRQ (bottom right, excluding requirements on $E_{\mathrm{T}}^{\text {miss }} / \sqrt{H_{\mathrm{T}}}$ ) corresponding to SRs $2 \mathrm{jl}, 2 \mathrm{jm}$ and $2 \mathrm{jt}$. With the exception of the multi-jet background (which is estimated using the data-driven technique described in the text), the histograms denote the MC background expectations, normalised to cross-section times integrated luminosity. In the lower panels the light (yellow) error bands denote the experimental systematic and MC statistical uncertainties, while the medium dark (green) bands include also the theoretical modelling uncertainty. The arrows indicate the values at which the requirements on $m_{\mathrm{eff}}$ (incl.) are applied.

\section{Background estimation}

\subsection{Overview}

The observed numbers of events in the CRs for each SR are used to generate consistent SM background estimates for the SR via a likelihood fit [98]. This procedure enables CR correlations due to common systematic uncertainties and contamination by other SM processes and/or SUSY signal events to be taken into account. Poisson likelihood functions are used for event counts in signal and control regions. Systematic uncertainties are treated 

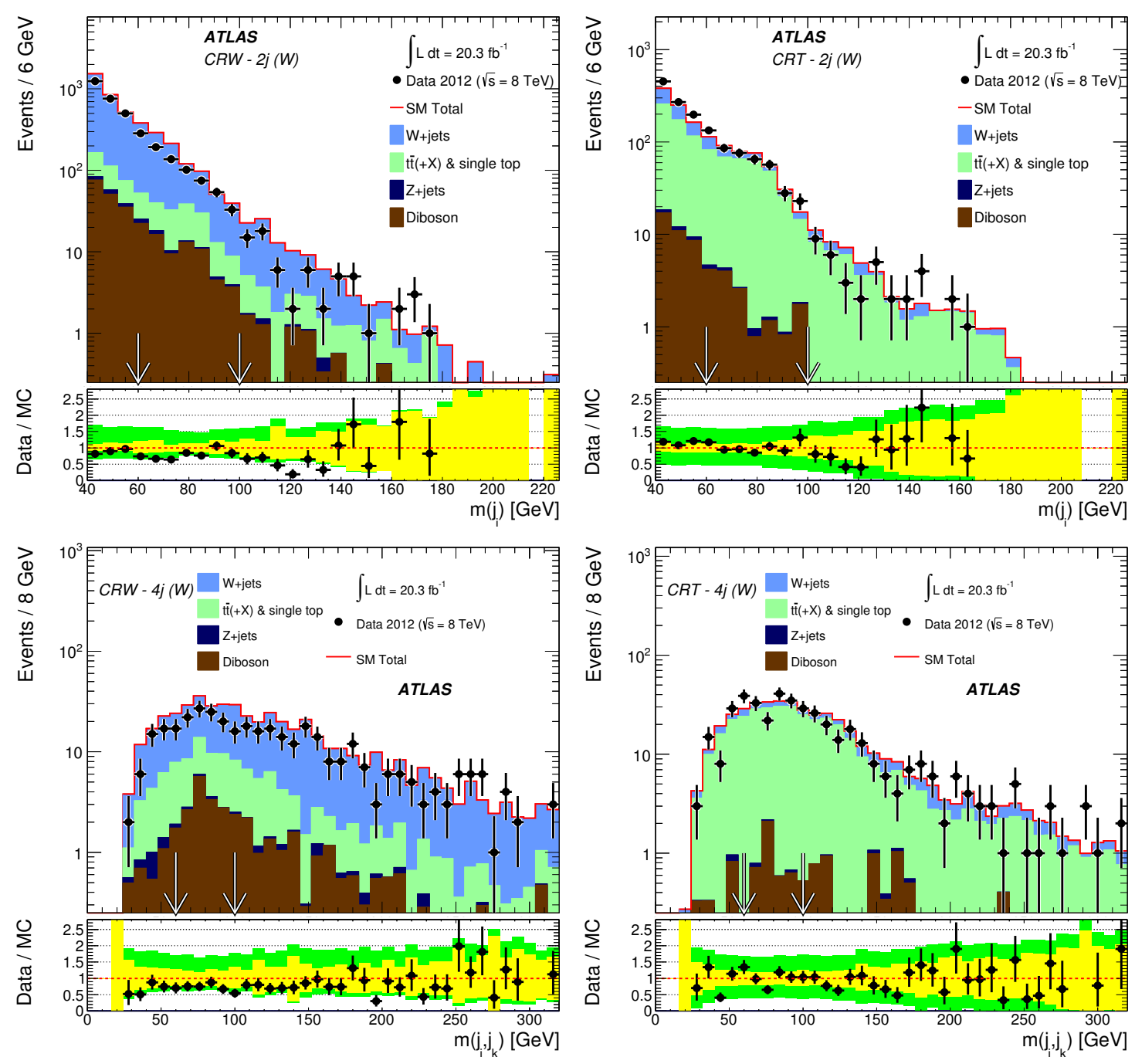

Figure 2. Observed jet (top) or dijet (bottom) mass distributions for the CRW (left) and CRT (right) selections for the $2 \mathrm{jW}$ (top) and $4 \mathrm{jW}$ (bottom) signal regions. In the case of the dijet mass distributions for $\mathrm{SR} 4 \mathrm{jW}$ (bottom), events are required to possess at least one unresolved $W$ candidate, with the dijet mass calculated from jets excluding the unresolved $W$ candidate. With the exception of the multi-jet background (which is estimated using the data-driven technique described in the text), the histograms denote the MC background expectations, normalised to cross-section times integrated luminosity. In the lower panels the light (yellow) error bands denote the experimental systematic and MC statistical uncertainties, while the medium dark (green) bands include also the theoretical modelling uncertainty.

as Gaussian-distributed nuisance parameters in the likelihood function. Key ingredients in the fit are the ratios of expected event counts from each background process between the SR and each CR, and between CRs. These ratios, referred to as transfer factors or 'TFs', enable observations in the CRs to be converted into background estimates in the SR using:

$$
N(\mathrm{SR}, \text { scaled })=N(\mathrm{CR}, \text { obs }) \times\left[\frac{N(\mathrm{SR}, \text { unscaled })}{N(\mathrm{CR}, \text { unscaled })}\right]
$$


where $N(\mathrm{SR}$, scaled) is the estimated background contribution to the SR by a given process, $N(\mathrm{CR}$, obs $)$ is the observed number of data events in the $\mathrm{CR}$ for the process, and $N(\mathrm{SR}$, unscaled) and $N(\mathrm{CR}$, unscaled) are a priori estimates of the contributions from the process to the $\mathrm{SR}$ and $\mathrm{CR}$, respectively. The TF is the ratio in the square brackets in eq. (7.1). Similar equations containing inter-CR TFs enable the background estimates to be normalised coherently across all the CRs associated with a given SR.

Background estimation requires determination of the central expected values of the TFs for each SM process, together with their associated correlated and uncorrelated uncertainties. Some systematic uncertainties, for instance those arising from the jet energy scale (JES), or theoretical uncertainties in MC cross-sections, largely cancel when calculating the event-count ratios constituting the TFs. The use of similar kinematic selections for the CRs and the SR minimises residual uncertainties correlated between these regions. The multi-jet TFs are estimated using a data-driven technique [16], which applies a resolution function to well-measured multi-jet events in order to estimate the impact of jet energy mismeasurement and heavy-flavour semileptonic decays on $E_{\mathrm{T}}^{\text {miss }}$ and other variables. The other TF estimates use MC samples. Corrections are applied to the CR $\gamma$ TFs which reduce the theoretical uncertainties in the SR $Z / \gamma^{*}+$ jets background expectations arising from the use of LO $\gamma+$ jets cross-sections (see table 1 ) when evaluating the denominator of the TF ratio in eq. (7.1). These corrections are determined by comparing CR $\gamma$ observations with observations in a highly populated auxiliary control region selecting events containing a low $p_{\mathrm{T}} Z$ boson $\left(160 \mathrm{GeV} \lesssim p_{\mathrm{T}}(Z) \lesssim 300 \mathrm{GeV}\right)$ decaying to electrons or muons, together with at least two jets.

Three different classes of likelihood fit are employed in this analysis. The first is used to determine the compatibility of the observed event yield in each SR with the corresponding SM background expectation. In this case (the 'background-only fit') the fit is performed using only the observed event yields from the CRs associated with the SR, but not the SR itself, as constraints. It is assumed that signal events from physics beyond standard model (BSM) do not contribute to these yields. The significance of an excess of events observed in the SR above the resulting SM background expectation is quantified by the probability (the one-sided p-value, $p_{0}$ ) that the SR event yield obtained in a single hypothetical backgroundonly experiment is greater than that observed in this dataset. The background-only fit is also used to estimate the background event yields in the VRs.

If no excess is observed, then a second class of likelihood fit (the 'model-independent fit') is used to set 'model-independent' upper limits on the number of BSM signal events in each SR. These limits, when normalised by the integrated luminosity of the data sample, may be interpreted as upper limits on the visible cross-section of BSM physics $(\langle\epsilon \sigma\rangle)$ defined as the product of acceptance, reconstruction efficiency and production cross-section. The model-independent fit proceeds in the same way as the background-only fit, except that the number of events observed in the SR is added as an input to the fit and the BSM signal strength, constrained to be non-negative, is added as a free parameter. Possible contamination of the CRs by BSM signal events is neglected.

A third class of likelihood fit (the 'SUSY-model exclusion fit') is used to set limits on the signal cross-sections for specific SUSY models. The SUSY-model exclusion fit proceeds 
in the same way as the model-independent fit, except that signal contamination in the $\mathrm{CR}$ is taken into account as well as theoretical and experimental uncertainties on the SUSY production cross-section and kinematic distributions. Correlations between signal and background systematic uncertainties are also taken into account where appropriate.

\subsection{Systematic uncertainties}

Systematic uncertainties in background estimates arise through the use of the transfer factors relating observations in the control regions to background expectations in the signal regions, and from the MC modelling of minor backgrounds. The total background uncertainties for all SRs, broken down into the main contributing sources, are presented in table 4. The overall background uncertainties range from $5 \%$ in SR 4jl-, where the loose selection minimises theoretical uncertainties and the impact of statistical fluctuations in the CRs, to $61 \%$ in $\mathrm{SR} 2 \mathrm{jW}$, where the opposite is true.

For the backgrounds estimated with MC simulation-derived transfer factors the primary common sources of systematic uncertainty are the jet energy scale (JES) calibration, jet energy resolution (JER), theoretical uncertainties, MC and CR data statistics and the reconstruction performance in the presence of pile-up. Correlations between uncertainties (for instance between JES uncertainties in CRs and SRs) are taken into account where appropriate.

The JES uncertainty was measured using the techniques described in refs. [82, 99], leading to a slight dependence upon $p_{\mathrm{T}}$ and $\eta$. The JER uncertainty is estimated using the methods discussed in ref. [100]. Contributions are added to both the JES and the JER uncertainties to account for the effect of pile-up at the relatively high luminosity delivered by the LHC in the 2012 run. A further uncertainty on the low- $p_{\mathrm{T}}$ calorimeter activity not associated with jets or baseline leptons but included in the $E_{\mathrm{T}}^{\text {miss }}$ calculation is taken into account. The jet mass scale and resolution uncertainties applicable in SR 2jW and SR $4 \mathrm{jW}$ are estimated using a sample of tagged $W \rightarrow q q^{\prime}$ decays reconstructed as single jets in selected $t \bar{t}$ events. For the specific selections used in these SRs these uncertainties are estimated to be of order $10 \%$ (scale) and $20 \%$ (resolution). The JES, JER, $E_{\mathrm{T}}^{\text {miss }}$ and jet mass scale and resolution (SR $2 \mathrm{jW}$ and $\mathrm{SR} 4 \mathrm{jW}$ ) uncertainties are taken into account in the combined 'Jet/MET' uncertainty quoted in table 4 . This uncertainty ranges from less than $1 \%$ of the expected background in SR $4 \mathrm{jm}$ to $12 \%$ in SR $2 \mathrm{jW}$.

Uncertainties arising from theoretical models of background processes are evaluated by comparing TFs obtained from samples produced with different MC generators, as described in section 4. Renormalisation and factorisation scale uncertainties are also taken into account by increasing and decreasing the scales used in the MC generators by a factor of two. The largest uncertainties are associated with the modelling of top quark production $(t \bar{t}$ and single top quark production) in the higher jet-multiplicity SRs (e.g. SR 4jW), and with the modelling of $Z / \gamma^{*}+$ jets in SR 4jt. Uncertainties associated with PDF modelling for background processes were checked with dedicated MC samples and found to be negligible. Uncertainties on diboson production due to scale and PDF errors are found to be $\lesssim 50 \%$ for all SRs, and a conservative uniform 50\% uncertainty is applied. The uncertainty on diboson production arising from the error on the integrated luminosity of the data sample 


\begin{tabular}{|c|c|c|c|c|c|}
\hline Channel & $2 \mathrm{jl}$ & 2jm & $2 \mathrm{jt}$ & $2 \mathrm{jW}$ & $\mathbf{3 j}$ \\
\hline Total bkg & 13000 & 760 & 125 & 2.3 & 5.0 \\
\hline Total bkg unc. & $\pm 1000[8 \%]$ & $\pm 50[7 \%]$ & $\pm 10[8 \%]$ & $\pm 1.4[61 \%]$ & $\pm 1.2[24 \%]$ \\
\hline CR stats: $Z / \gamma^{*}+$ jets & $\pm 100[0.8 \%]$ & $\pm 15[2.0 \%]$ & $\pm 5[4.0 \%]$ & $\pm 0.4[17.4 \%]$ & $\pm 0.7[14.0 \%]$ \\
\hline CR stats: $W+$ jets & $\pm 300[2.3 \%]$ & $\pm 21[2.8 \%]$ & $\pm 5[4.0 \%]$ & $\pm 0.7[30.4 \%]$ & $\pm 0.8[16.0 \%]$ \\
\hline CR stats: top quark & $\pm 200[1.5 \%]$ & $\pm 5[0.7 \%]$ & $\pm 1.6[1.3 \%]$ & $\pm 0.35[15.2 \%]$ & $\pm 0.5[10.0 \%]$ \\
\hline CR stats: multi-jets & - & - & $\pm 0.1[0.1 \%]$ & - & $\pm 0.1[2.0 \%]$ \\
\hline MC statistics & $\pm 130[1.0 \%]$ & $\pm 6[0.8 \%]$ & $\pm 2.1[1.7 \%]$ & $\pm 0.34[14.8 \%]$ & $\pm 0.35[7.0 \%]$ \\
\hline Jet/MET & $\pm 140[1.1 \%]$ & $\pm 8[1.1 \%]$ & $\pm 0.7[0.6 \%]$ & $\pm 0.27[11.7 \%]$ & $\pm 0.23[4.6 \%]$ \\
\hline Leptons & $\pm 80[0.6 \%]$ & $\pm 2.5[0.3 \%]$ & $\pm 0.6[0.5 \%]$ & $\pm 0.04[1.7 \%]$ & $\pm 0.06[1.2 \%]$ \\
\hline$Z / \gamma \mathrm{TF}$ & $\pm 500[3.8 \%]$ & $\pm 35[4.6 \%]$ & $\pm 5[4.0 \%]$ & $\pm 0.028[1.2 \%]$ & $\pm 0.14[2.8 \%]$ \\
\hline Theory: $Z / \gamma^{*}+$ jets & $\pm 800[6.2 \%]$ & $\pm 5[0.7 \%]$ & $\pm 4[3.2 \%]$ & $\pm 0.03[1.3 \%]$ & $\pm 0.29[5.8 \%]$ \\
\hline Theory: $W+$ jets & $\pm 270[2.1 \%]$ & $\pm 10[1.3 \%]$ & $\pm 1.4[1.1 \%]$ & $\pm 0.1[4.3 \%]$ & $\pm 0.35[7.0 \%]$ \\
\hline Theory: top quark & $\pm 13[0.1 \%]$ & $\pm 1.8[0.2 \%]$ & $\pm 0.11[0.1 \%]$ & $\pm 0.9[39.1 \%]$ & $\pm 0.05[1.0 \%]$ \\
\hline Theory: diboson & $\pm 400[3.1 \%]$ & $\pm 40[5.3 \%]$ & $\pm 6[4.8 \%]$ & $\pm 0.2[8.7 \%]$ & $\pm 0.18[3.6 \%]$ \\
\hline Theory: scale unc. & $\pm 90[0.7 \%]$ & $\pm 4[0.5 \%]$ & $\pm 0.7[0.6 \%]$ & $\pm 0.13[5.7 \%]$ & $\pm 0.12[2.4 \%]$ \\
\hline Multi-jets method & $\pm 140[1.1 \%]$ & $\pm 1.4[0.2 \%]$ & $\pm 0.4[0.3 \%]$ & $\pm 0.04[1.7 \%]$ & $\pm 0.06[1.2 \%]$ \\
\hline Other & $\pm 32[0.2 \%]$ & $\pm 0.6[0.1 \%]$ & $\pm 0.4[0.3 \%]$ & $\pm 0.24[10.4 \%]$ & $\pm 0.02[0.4 \%]$ \\
\hline Channel & $4 \mathrm{jl}-$ & $4 \mathrm{jl}$ & $4 \mathrm{jm}$ & $4 \mathrm{jt}$ & $4 \mathrm{jW}$ \\
\hline Total bkg & 2120 & 630 & 37 & 2.5 & 14 \\
\hline Total bkg unc. & $\pm 110[5 \%]$ & $\pm 50[8 \%]$ & $\pm 6[16 \%]$ & $\pm 1.0[40 \%]$ & $\pm 4[29 \%]$ \\
\hline CR stats: $Z / \gamma^{*}+$ jets & $\pm 22[1.0 \%]$ & $\pm 12[1.9 \%]$ & $\pm 2.3[6.2 \%]$ & $\pm 0.5[20.0 \%]$ & $\pm 1.3[9.3 \%]$ \\
\hline CR stats: $W+$ jets & $\pm 60[2.8 \%]$ & $\pm 25[4.0 \%]$ & $\pm 1.3[3.5 \%]$ & $\pm 0.4[16.0 \%]$ & $\pm 1.0[7.1 \%]$ \\
\hline CR stats: top quark & $\pm 40[1.9 \%]$ & $\pm 16[2.5 \%]$ & $\pm 0.5[1.4 \%]$ & $\pm 0.4[16.0 \%]$ & $\pm 0.5[3.6 \%]$ \\
\hline CR stats: multi-jets & - & - & - & - & - \\
\hline MC statistics & $\pm 18[0.8 \%]$ & $\pm 6[1.0 \%]$ & $\pm 1.3[3.5 \%]$ & $\pm 0.26[10.4 \%]$ & $\pm 0.7[5.0 \%]$ \\
\hline Jet/MET & $\pm 40[1.9 \%]$ & $\pm 7[1.1 \%]$ & $\pm 0.15[0.4 \%]$ & $\pm 0.06[2.4 \%]$ & $\pm 0.6[4.3 \%]$ \\
\hline Leptons & $\pm 20[0.9 \%]$ & $\pm 5[0.8 \%]$ & $\pm 0.27[0.7 \%]$ & $\pm 0.08[3.2 \%]$ & $\pm 0.06[0.4 \%]$ \\
\hline$Z / \gamma \mathrm{TF}$ & $\pm 50[2.4 \%]$ & $\pm 19[3.0 \%]$ & $\pm 1.3[3.5 \%]$ & $\pm 0.06[2.4 \%]$ & $\pm 0.5[3.6 \%]$ \\
\hline Theory: $Z / \gamma^{*}+$ jets & - & $\pm 18[2.9 \%]$ & $\pm 2.4[6.5 \%]$ & $\pm 0.4[16.0 \%]$ & $\pm 1.3[9.3 \%]$ \\
\hline Theory: $W+$ jets & $\pm 33[1.6 \%]$ & $\pm 7[1.1 \%]$ & $\pm 2.3[6.2 \%]$ & $\pm 0.07[2.8 \%]$ & $\pm 0.9[6.4 \%]$ \\
\hline Theory: top quark & $\pm 29[1.4 \%]$ & $\pm 12[1.9 \%]$ & $\pm 1.6[4.3 \%]$ & $\pm 0.4[16.0 \%]$ & $\pm 2.8[20.0 \%]$ \\
\hline Theory: diboson & $\pm 90[4.2 \%]$ & $\pm 35[5.6 \%]$ & $\pm 4[10.8 \%]$ & $\pm 0.17[6.8 \%]$ & $\pm 1.0[7.1 \%]$ \\
\hline Theory: scale unc. & $\pm 23[1.1 \%]$ & $\pm 7[1.1 \%]$ & $\pm 0.4[1.1 \%]$ & $\pm 0.13[5.2 \%]$ & $\pm 0.12[0.9 \%]$ \\
\hline Multi-jets method & $\pm 4[0.2 \%]$ & $\pm 1.6[0.3 \%]$ & - & - & -1 \\
\hline Other & $\pm 5[0.2 \%]$ & $\pm 5[0.8 \%]$ & $\pm 0.23[0.6 \%]$ & $\pm 0.06[2.4 \%]$ & $\pm 0.12[0.9 \%]$ \\
\hline Channel & $5 \mathbf{j}$ & $6 \mathrm{jl}$ & $6 \mathrm{jm}$ & $6 \mathrm{jt}$ & $6 \mathrm{jt}+$ \\
\hline Total bkg & 126 & 111 & 33 & 5.2 & 4.9 \\
\hline Total bkg unc. & $\pm 13[10 \%]$ & $\pm 11[10 \%]$ & $\pm 6[18 \%]$ & $\pm 1.4[27 \%]$ & $\pm 1.6[33 \%]$ \\
\hline CR stats: $Z / \gamma^{*}+$ jets & $\pm 3.0[2.4 \%]$ & $\pm 1.4[1.3 \%]$ & $\pm 0.7[2.1 \%]$ & $\pm 0.33[6.3 \%]$ & $\pm 0.31[6.3 \%]$ \\
\hline CR stats: $W+$ jets & $\pm 6[4.8 \%]$ & $\pm 4[3.6 \%]$ & $\pm 2.4[7.3 \%]$ & $\pm 0.5[9.6 \%]$ & $\pm 0.7[14.3 \%]$ \\
\hline CR stats: top quark & $\pm 7[5.6 \%]$ & $\pm 7[6.3 \%]$ & $\pm 2.3[7.0 \%]$ & $\pm 0.31[6.0 \%]$ & $\pm 1.1[22.4 \%]$ \\
\hline CR stats: multi-jets & $\pm 0.08[0.1 \%]$ & $\pm 0.19[0.2 \%]$ & $\pm 0.08[0.2 \%]$ & - & $\pm 0.04[0.8 \%]$ \\
\hline MC statistics & $\pm 2.8[2.2 \%]$ & $\pm 2.8[2.5 \%]$ & $\pm 1.5[4.5 \%]$ & $\pm 0.7[13.5 \%]$ & $\pm 0.4[8.2 \%]$ \\
\hline Jet/MET & $\pm 4[3.2 \%]$ & $\pm 6[5.4 \%]$ & $\pm 1.2[3.6 \%]$ & $\pm 0.5[9.6 \%]$ & $\pm 0.29[5.9 \%]$ \\
\hline Leptons & $\pm 1.8[1.4 \%]$ & $\pm 1.8[1.6 \%]$ & $\pm 0.7[2.1 \%]$ & $\pm 0.05[1.0 \%]$ & $\pm 0.32[6.5 \%]$ \\
\hline$Z / \gamma \mathrm{TF}$ & $\pm 2.5[2.0 \%]$ & $\pm 0.8[0.7 \%]$ & $\pm 0.27[0.8 \%]$ & $\pm 0.04[0.8 \%]$ & $\pm 0.04[0.8 \%]$ \\
\hline Theory: $Z / \gamma^{*}+$ jets & $\pm 7[5.6 \%]$ & $\pm 3.0[2.7 \%]$ & $\pm 2.0[6.1 \%]$ & $\pm 0.5[9.6 \%]$ & $\pm 0.7[14.3 \%]$ \\
\hline Theory: $W+$ jets & $\pm 2.2[1.7 \%]$ & $\pm 1.7[1.5 \%]$ & $\pm 2.8[8.5 \%]$ & $\pm 0.4[7.7 \%]$ & $\pm 0.08[1.6 \%]$ \\
\hline Theory: top quark & $\pm 5[4.0 \%]$ & $\pm 2.7[2.4 \%]$ & $\pm 3.5[10.6 \%]$ & $\pm 0.08[1.5 \%]$ & $\pm 0.5[10.2 \%]$ \\
\hline Theory: diboson & $\pm 8[6.3 \%]$ & $\pm 4[3.6 \%]$ & $\pm 1.9[5.8 \%]$ & $\pm 0.8[15.4 \%]$ & $\pm 0.1[2.0 \%]$ \\
\hline Theory: scale unc. & $\pm 2.5[2.0 \%]$ & $\pm 1.1[1.0 \%]$ & $\pm 0.8[2.4 \%]$ & $\pm 0.11[2.1 \%]$ & $\pm 0.5[10.2 \%]$ \\
\hline Multi-jets method & $\pm 2.6[2.1 \%]$ & $\pm 2.9[2.6 \%]$ & $\pm 0.8[2.4 \%]$ & $\pm 0.032[0.6 \%]$ & $\pm 0.4[8.2 \%]$ \\
\hline Other & $\pm 0.9[0.7 \%]$ & $\pm 2.5[2.3 \%]$ & $\pm 0.9[2.7 \%]$ & $\pm 0.14[2.7 \%]$ & $\pm 0.03[0.6 \%]$ \\
\hline
\end{tabular}

Table 4. Breakdown of the systematic uncertainties on background estimates obtained from the fits described in the text. Note that the individual uncertainties can be correlated, and do not necessarily sum in quadrature to the total background uncertainty. Uncertainties relative to the total expected background yield are shown in parenthesis. When a dash is shown, the resulting relative uncertainty is lower than $0.1 \%$. Rows labelled 'CR stats' refer to uncertainties arising from finite data statistics in the main CR for the background process specified. 
is negligible, while for other processes this uncertainty cancels in the TF ratio between CR and SR event yields.

The statistical uncertainty arising from the use of finite-size MC samples is largest $(15 \%)$ in $\mathrm{SR} 2 \mathrm{jW}$. Uncertainties arising from finite data statistics in the control regions are most important for the tighter signal region selections, reaching $20 \%$ for $Z / \gamma^{*}+$ jets (estimated with $\mathrm{CR} \gamma$ ) in SR $4 \mathrm{jt}$ and $22 \%$ for top quark production processes (estimated with CRT) in SR 6jt+.

The experimental systematic uncertainties associated with CR event reconstruction include photon and lepton reconstruction efficiency, energy scale and resolution (CR $\gamma$, CRW and CRT) and $b$-tag/ $b$-veto efficiency (CRW and CRT). The photon reconstruction uncertainties associated with $\mathrm{CR} \gamma$, together with uncertainties arising from the data-driven $\mathrm{CR} \gamma \mathrm{TF}$ correction procedure described in section 7.1 , are included in table 4 under ' $Z / \gamma$ TF'. The impact of lepton reconstruction uncertainties on the overall background uncertainty is found to be negligible for all SRs. Uncertainties in the $b$-tag/ $b$-veto efficiency are included in table 4 under 'other', together with additional small uncertainties such as those associated with the modelling of pile-up in MC events.

Uncertainties related to the multi-jet background estimates are determined by varying the width and tails of the jet resolution function within the appropriate experimental uncertainties and then repeating the background estimation procedure described in section 7.1. The maximum resulting contribution to the overall background uncertainty is $8 \%$ in SR $6 \mathrm{jt}+$.

\subsection{Validation}

The background estimation procedure is validated by comparing the numbers of events observed in the VRs (see section 6.3) in the data to the corresponding SM background expectations obtained from the background-only fits. The results are shown in figure 3 . The entries in the table are the differences between the numbers of observed and expected events expressed as fractions of the one-standard deviation $(1 \sigma)$ uncertainties on the latter. Most VR observations lie within $1 \sigma$ of the background expectations, with the largest discrepancy out of the 135 VRs being $2.4 \sigma$ (13 events observed, $6.1 \pm 1.3$ expected) for the VRZ region associated with SR $5 \mathrm{j}$.

\section{Results}

Distributions of $m_{\mathrm{eff}}$ (incl.) and jet and dijet masses (the latter for SR $2 \mathrm{jW}$ and SR $4 \mathrm{jW}$ ) obtained before the final selections on these quantities (but after applying all other selections), for data and the different MC samples normalised with the theoretical cross-sections (with the exception of the multi-jet background, which is estimated using the data-driven technique described in section 7.1), are shown in figures 4-6. Examples of typical expected SUSY signals are shown for illustration. These signals correspond to the processes to which each SR is primarily sensitive - $\tilde{q} \tilde{q}$ production for the lower jet-multiplicity SRs, $\tilde{q} \tilde{g}$ associated production for intermediate jet-multiplicity SRs, and $\tilde{g} \tilde{g}$ production for the higher jet-multiplicity SRs. In these figures data and background distributions largely 


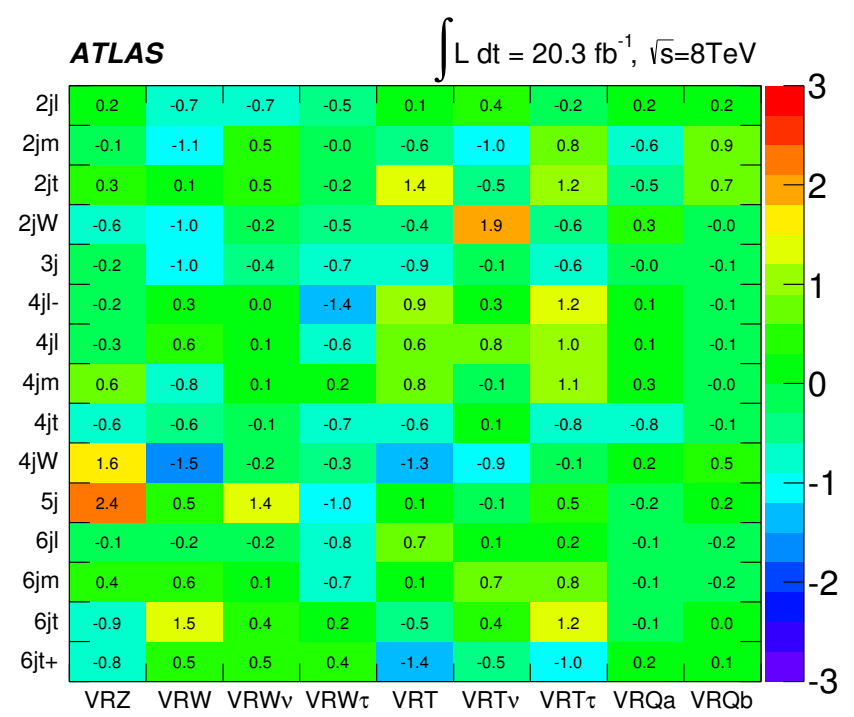

Figure 3. Differences between the numbers of observed events in data and SM background expectations for each VR, expressed as fractions of the uncertainties on the latter.

agree within uncertainties; however, there is a systematic difference between the data and the background prediction which increases towards larger values of the kinematic variables considered. This difference does not affect the background expectations in the signal regions used in the analysis, however, due to the use of the likelihood fits to the CR event yields discussed in section 7.1.

The number of events observed in the data and the number of SM events expected to enter each of the signal regions, determined using the background-only fit, are shown in table 5 and figure 7 . The pre-fit background expectations are also shown in table 5 to aid comparison. The fit to the CRs for each SR compensates for the disagreement between data and pre-fit background expectations seen in figures 4-6, leading to good agreement between data and post-fit expectations. The most significant observed excess across the 15 SRs, with a p-value for the background-only hypothesis of 0.24 , occurs in SR 3j.

\section{Interpretation}

In the absence of a statistically significant excess, limits are set on contributions to the SRs from BSM physics. Upper limits at 95\% CL on the number of BSM signal events in each SR and the corresponding visible BSM cross-section are derived from the modelindependent fits described in section 7.1 using the $\mathrm{CL}_{\mathrm{s}}$ prescription [101]. The limits are evaluated using MC pseudo-experiments as well as asymptotic formulae [98]. The results are presented in table 5. Asymptotic limits differ appreciably from those evaluated using $\mathrm{MC}$ pseudo-experiments only for the tightest signal regions $(2 \mathrm{jW}$ and $4 \mathrm{jt})$, where the small expected number of events limits the accuracy of the former.

The SUSY-model exclusion fits in all the SRs are then used to set limits on specific classes of SUSY models, using the result from the SR with the best expected sensitivity at each point in each model parameter space. These limits are evaluated using asymptotic 

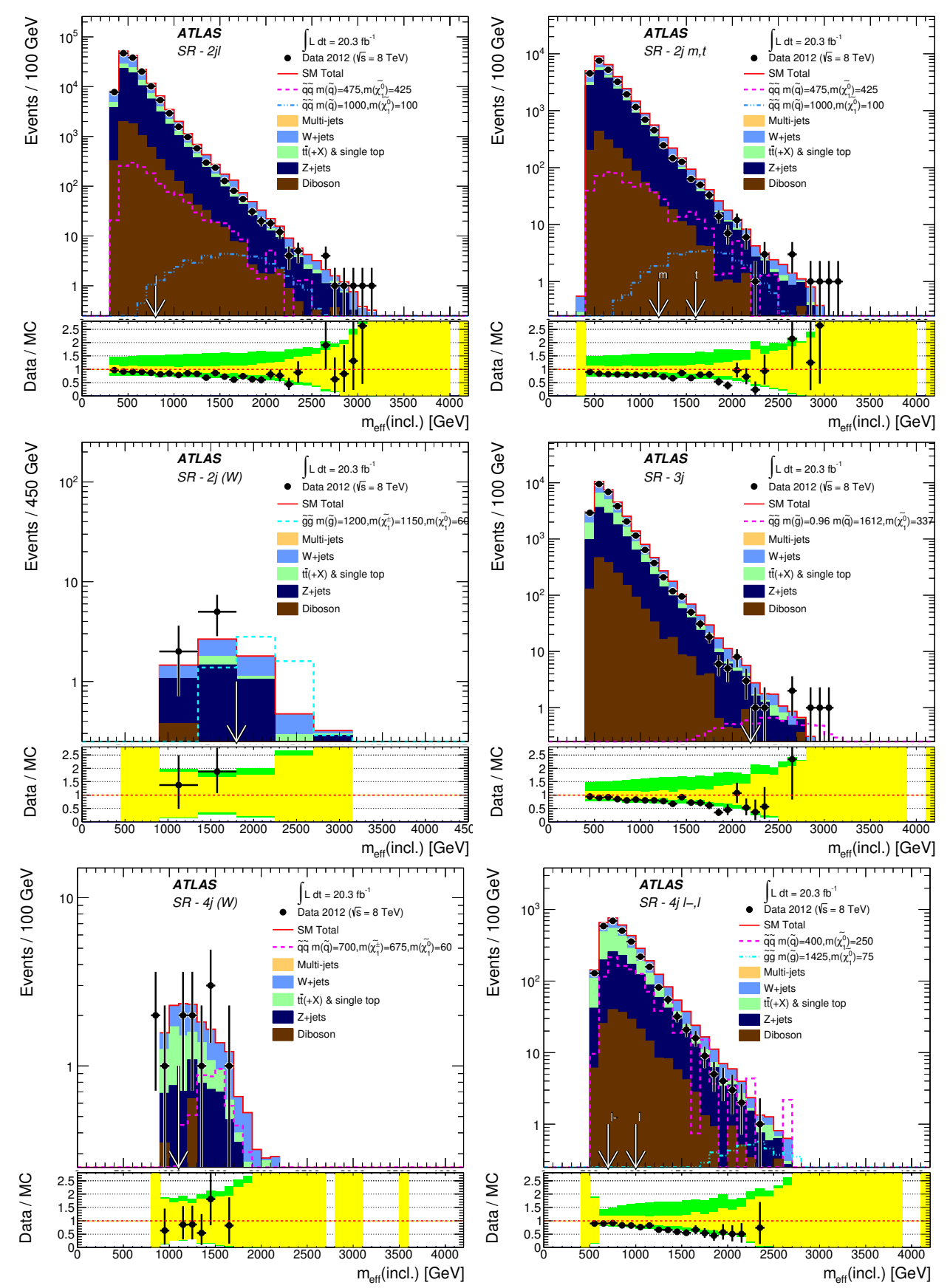

Figure 4. Observed $m_{\mathrm{eff}}$ (incl.) distributions for the 2-jet (top and middle-left), 3-jet (middleright) and 4-jet (4jW, 4jl- and 4jl) signal regions (bottom). With the exception of the multi-jet background (which is estimated using the data-driven technique described in the text), the histograms denote the MC background expectations prior to the fits described in the text, normalised to cross-section times integrated luminosity. In the lower panels the light (yellow) error bands denote the experimental systematic and MC statistical uncertainties, while the medium dark (green) bands include also the theoretical modelling uncertainty. The arrows indicate the values at which the requirements on $m_{\text {eff }}$ (incl.) are applied. Expected distributions for benchmark model points are also shown for comparison (masses in $\mathrm{GeV}$ ). See text for discussion of compatibility of data with $\mathrm{MC}$ background expectations. 

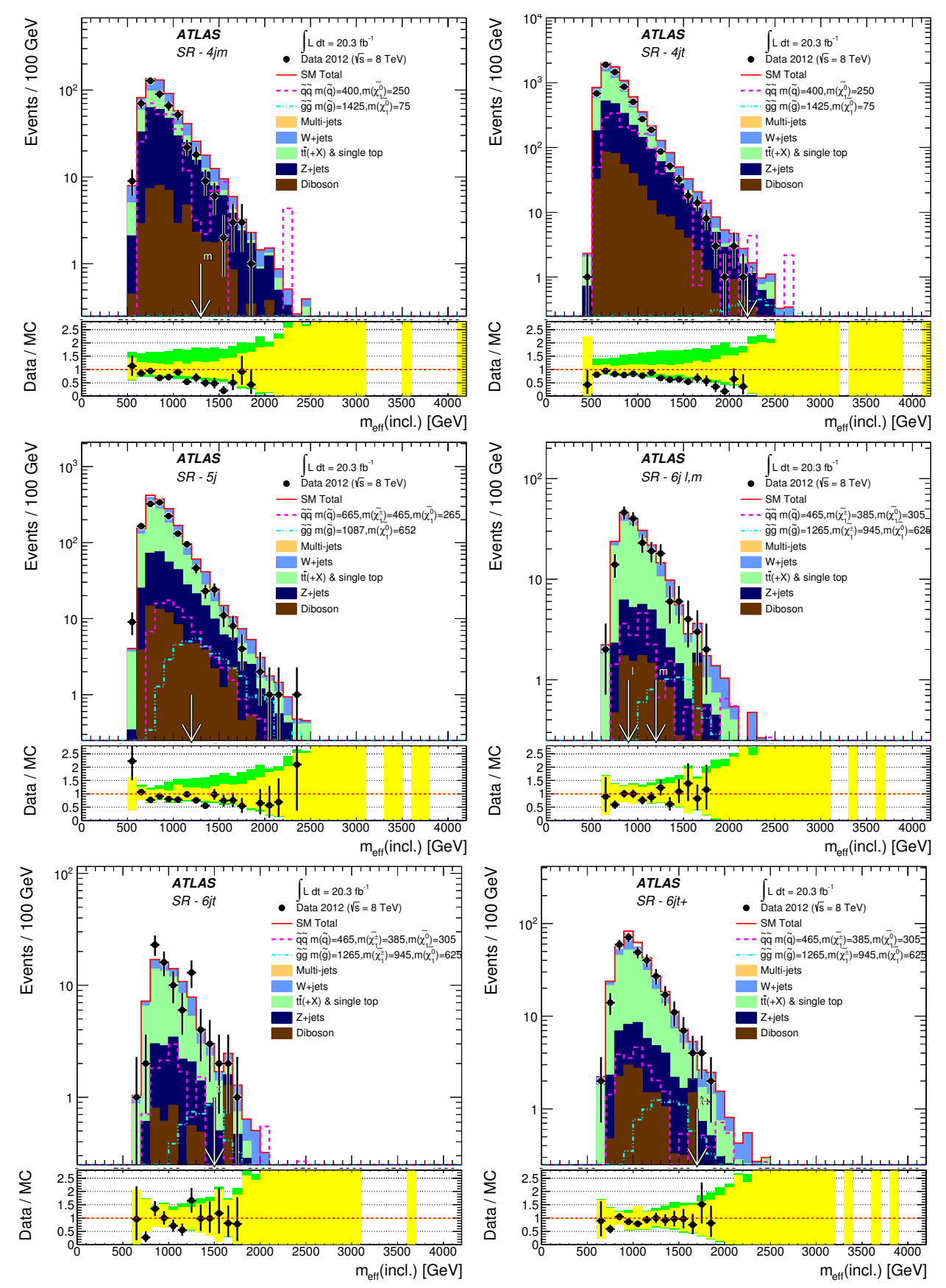

Figure 5. Observed $m_{\text {eff }}$ (incl.) distributions for the medium and tight 4-jet (top), 5-jet (middleleft) and 6-jet (middle-right and bottom) signal regions. With the exception of the multi-jet background (which is estimated using the data-driven technique described in the text), the histograms denote the MC background expectations prior to the fits described in the text, normalised to crosssection times integrated luminosity. In the lower panels the light (yellow) error bands denote the experimental systematic and MC statistical uncertainties, while the medium dark (green) bands include also the theoretical modelling uncertainty. The arrows indicate the values at which the requirements on $m_{\mathrm{eff}}$ (incl.) are applied. Expected distributions for benchmark model points are also shown for comparison (masses in $\mathrm{GeV}$ ). See text for discussion of compatibility of data with $\mathrm{MC}$ background expectations. 


\begin{tabular}{|c|c|c|c|c|c|}
\hline Signal Region & $2 \mathrm{jl}$ & $2 \mathrm{jm}$ & $2 \mathrm{jt}$ & $2 \mathrm{jW}$ & $3 \mathbf{j}$ \\
\hline \multicolumn{6}{|c|}{ MC expected events } \\
\hline Diboson & 879 & 72 & 13 & 0.41 & 0.36 \\
\hline$Z / \gamma^{*}+$ jets & 6709 & 552 & 103 & 1.2 & 5.5 \\
\hline$W+$ jets & 5472 & 303 & 59 & 0.82 & 3.1 \\
\hline$t \bar{t}(+\mathrm{EW})+$ single top & 1807 & 54 & 9 & 0.14 & 0.85 \\
\hline \multicolumn{6}{|c|}{ Fitted background events } \\
\hline Diboson & $900 \pm 400$ & $70 \pm 40$ & $13 \pm 6$ & $0.41 \pm 0.21$ & $0.36 \pm 0.18$ \\
\hline$Z / \gamma^{*}+$ jets & $5900 \pm 900$ & $430 \pm 40$ & $65 \pm 8$ & $0.4 \pm 0.4$ & $1.7 \pm 1.0$ \\
\hline$W+$ jets & $4500 \pm 600$ & $216 \pm 26$ & $40 \pm 6$ & $1.0 \pm 1.0$ & $2.5 \pm 0.9$ \\
\hline$t \bar{t}(+\mathrm{EW})+$ single top & $1620 \pm 320$ & $47 \pm 8$ & $6.5 \pm 2.2$ & $0.4_{-0.4}^{+0.8}$ & $0.4_{-0.4}^{+0.5}$ \\
\hline Multi-jets & $115_{-120}^{+140}$ & $0.4_{-0.4}^{+1.4}$ & $0.1_{-0.1}^{+0.4}$ & $0.03 \pm 0.03$ & $0.03_{-0.03}^{+0.06}$ \\
\hline Total bkg & $13000 \pm 1000$ & $760 \pm 50$ & $125 \pm 10$ & $2.3 \pm 1.4$ & $5.0 \pm 1.2$ \\
\hline Observed & 12315 & 715 & 133 & 0 & 7 \\
\hline$\langle\epsilon \sigma\rangle_{\mathrm{obs}}^{95}[\mathrm{fb}]$ & 60 & 4.3 & 1.9 & 0.16 & 0.40 \\
\hline$\langle\epsilon \sigma\rangle_{\mathrm{obs}}^{95}[\mathrm{fb}]$ (asymptotic) & 62 & 4.0 & 1.8 & 0.12 & 0.40 \\
\hline$S_{\mathrm{obs}}^{95}$ & 1200 & 90 & 38 & 3.2 & 8.2 \\
\hline$S_{\text {obs }}^{95}$ (asymptotic) & 1300 & 80 & 37 & 2.5 & 8.1 \\
\hline$S_{\exp }^{95}$ & $1700_{-500}^{+600}$ & $110_{-30}^{+40}$ & $32_{-10}^{+11}$ & $4.0_{-0.7}^{+1.7}$ & $6.4_{-1.3}^{+2.9}$ \\
\hline$S_{\text {exp }}^{95}$ (asymptotic) & $1600_{-400}^{+600}$ & $110_{-30}^{+40}$ & $31_{-8}^{+12}$ & $4.1_{-1.4}^{+2.4}$ & $6.3_{-2.0}^{+3.2}$ \\
\hline$p_{0}(Z)$ & $0.50(0.0)$ & $0.49(0.0)$ & $0.29(0.5)$ & $0.50(0.0)$ & $0.24(0.7)$ \\
\hline Signal Region & $4 \mathrm{jl}-$ & $4 \mathrm{jl}$ & $4 \mathrm{jm}$ & $4 \mathrm{jt}$ & $4 \mathrm{jW}$ \\
\hline \multicolumn{6}{|c|}{ MC expected events } \\
\hline Diboson & 175 & 70 & 7.2 & 0.34 & 2.1 \\
\hline$Z / \gamma^{*}+$ jets & 885 & 333 & 30 & 2.9 & 11 \\
\hline$W+$ jets & 832 & 284 & 16 & 1.2 & 6.1 \\
\hline$t \bar{t}(+\mathrm{EW})+$ single top & 764 & 167 & 4.0 & 0.6 & 3.1 \\
\hline \multicolumn{6}{|c|}{ Fitted background events } \\
\hline Diboson & $180 \pm 90$ & $70 \pm 34$ & $7 \pm 4$ & $0.34 \pm 0.17$ & $2.1 \pm 1.0$ \\
\hline$Z / \gamma^{*}+$ jets & $660 \pm 60$ & $238 \pm 28$ & $16 \pm 4$ & $0.7_{-0.7}^{+0.8}$ & $5.9 \pm 2.1$ \\
\hline$W+$ jets & $560 \pm 80$ & $151 \pm 28$ & $10 \pm 4$ & $0.9 \pm 0.4$ & $2.7 \pm 1.6$ \\
\hline$t \bar{t}(+\mathrm{EW})+$ single top & $730 \pm 50$ & $167 \pm 18$ & $4 \pm 2$ & $0.6 \pm 0.6$ & $3.2 \pm 3.1$ \\
\hline Multi-jets & $1.7_{-1.7}^{+4.0}$ & $0.7_{-0.7}^{+1.6}$ & - & - & - \\
\hline Total bkg & $2120 \pm 110$ & $630 \pm 50$ & $37 \pm 6$ & $2.5 \pm 1.0$ & $14 \pm 4$ \\
\hline Observed & 2169 & 608 & 24 & 0 & 16 \\
\hline$\langle\epsilon \sigma\rangle_{\mathrm{obs}}^{95}[\mathrm{fb}]$ & 13 & 4.5 & 0.52 & 0.15 & 0.68 \\
\hline$\langle\epsilon \sigma\rangle_{\text {obs }}^{95}[\mathrm{fb}]$ (asymptotic) & 13 & 4.3 & 0.45 & 0.12 & 0.63 \\
\hline$S_{\text {obs }}^{95}$ & 270 & 91 & 10 & 3.1 & 14 \\
\hline$S_{\text {obs }}^{95}$ (asymptotic) & 270 & 87 & 9 & 2.5 & 13 \\
\hline$S_{\exp }^{95}$ & $240_{-70}^{+90}$ & $103_{-29}^{+34}$ & $16_{-4}^{+6}$ & $4.0_{-0.9}^{+1.8}$ & $11_{-3}^{+5}$ \\
\hline$S_{\exp }^{95}$ (asymptotic) & $240_{-70}^{+90}$ & $97_{-25}^{+35}$ & $15_{-4}^{+6}$ & $4.0_{-1.4}^{+2.4}$ & $11_{-3}^{+5}$ \\
\hline$p_{0}(Z)$ & $0.35(0.4)$ & $0.50(0.0)$ & $0.50(0.0)$ & $0.50(0.0)$ & $0.34(0.4)$ \\
\hline
\end{tabular}

Continued on next page... 


\begin{tabular}{|lrrrrr|}
\hline Signal Region & $\mathbf{5 j}$ & $\mathbf{6 j} \mathbf{j}$ & $\mathbf{6 j m}$ & $\mathbf{6 j t}$ & $\mathbf{6 j t +}$ \\
\hline \multicolumn{5}{c}{ MC expected events } \\
\hline Diboson & 16 & 9 & 4 & 1.6 & 0.21 \\
$Z / \gamma^{*}+$ jets & 51 & 18 & 7 & 1.8 & 2.1 \\
$W+$ jets & 54 & 26 & 12 & 2.1 & 3.4 \\
$t \bar{t}(+\mathrm{EW})+$ single top & 52 & 80 & 19 & 2.2 & 3.4 \\
\hline \multicolumn{5}{c}{} \\
\hline Diboson & Fitted background events \\
$Z / \gamma^{*}+$ jets & $16 \pm 8$ & $9 \pm 4$ & $4 \pm 2$ & $1.6 \pm 0.8$ & $0.2 \pm 0.1$ \\
$W+$ jets & $31 \pm 8$ & $9 \pm 4$ & $3 \pm 2$ & $0.6 \pm 0.6$ & $0.6_{-0.6}^{+0.8}$ \\
$t \bar{t}(+$ EW $)+$ single top & $28 \pm 8$ & $15 \pm 7$ & $9 \pm 5$ & $1.2 \pm 0.9$ & $0.3_{-0.3}^{+1.2}$ \\
Multi-jets & $51 \pm 9$ & $76 \pm 7$ & $16 \pm 4$ & $1.8 \pm 0.6$ & $3.7 \pm 1.7$ \\
\hline Total bkg & $1.0_{-1.0}^{+2.6}$ & $1.7_{-1.7}^{+3.0}$ & $0.4_{-0.4}^{+0.8}$ & $0.01_{-0.01}^{+0.03}$ & $0.3_{-0.3}^{+0.4}$ \\
\hline Observed & $126 \pm 13$ & $111 \pm 11$ & $33 \pm 6$ & $5.2 \pm 1.4$ & $4.9 \pm 1.6$ \\
\hline$\langle\epsilon \sigma\rangle_{\text {obs }}^{95}[\mathrm{fb}]$ & 121 & 121 & 39 & 5 & 6 \\
$\langle\epsilon \sigma\rangle_{\text {obs }}^{95}[\mathrm{fb}]$ (asymptotic) & 1.7 & 1.9 & 1.2 & 0.32 & 0.39 \\
$S_{\text {obs }}^{95}$ & 1.6 & 1.8 & 1.1 & 0.30 & 0.36 \\
$S_{\text {obs }}^{95}$ (asymptotic) & 35 & 39 & 25 & 6.6 & 7.9 \\
$S_{\text {exp }}^{95}$ & 32 & 37 & 22 & 6.1 & 7.3 \\
$S_{\text {exp }}^{95}$ (asymptotic) & $37_{-10}^{+13}$ & $31_{-6}^{+12}$ & $20_{-4}^{+6}$ & $6.2_{-1.3}^{+2.6}$ & $6.6_{-1.6}^{+2.6}$ \\
$p_{0}(Z)$ & $35_{-10}^{+13}$ & $30_{-8}^{+12}$ & $18_{-5}^{+7}$ & $6.3_{-2.0}^{+3.1}$ & $6.4_{-2.0}^{+3.2}$ \\
\hline
\end{tabular}

Table 5. Numbers of events observed in the signal regions used in the analysis compared with background expectations obtained from the fits described in the text. When a dash is shown, the entry is less than 0.01. Combined uncertainties on the predicted background event yields are quoted as symmetric except where the negative uncertainty reaches down to zero predicted events, in which case the negative uncertainty is truncated. The p-values $\left(p_{0}\right)$ for the background-only hypothesis are truncated at 0.5 and are also interpreted in terms of the equivalent Gaussian significance $(Z)$. Also shown are 95\% CL upper limits on the visible cross-section $\left(\langle\epsilon \sigma\rangle_{\text {obs }}^{95}\right)$, the observed number of signal events $\left(S_{\mathrm{obs}}^{95}\right)$ and the number of signal events $\left(S_{\exp }^{95}\right)$ given the expected number (and $\pm 1 \sigma$ excursions on the expectation) of background events. Limits are evaluated using MC pseudoexperiments as well as asymptotic formulae.

formulae only. 'Observed limits' are calculated from the observed SR event yields for both the nominal signal cross-section and with its $\pm 1 \sigma$ uncertainties. Numbers quoted in the text are evaluated in a conservative fashion from the observed exclusion limit based on the nominal signal cross-section minus its $1 \sigma$ theoretical uncertainty. 'Expected limits' are calculated by setting the nominal event yield in each SR to the corresponding mean expected background.

Theoretical uncertainties on both the signal cross-section (discussed in section 4) and signal acceptance are taken into account when setting limits on specific SUSY models using the SUSY-model exclusion fits. An important consideration is that initial state radiation (ISR) can significantly affect the signal acceptance for SUSY models with small mass splittings, $\Delta m$, between the strongly interacting states $(\tilde{q}$ or $\tilde{g})$ and the $\tilde{\chi}_{1}^{0}$. Systematic uncertainties arising from the treatment of ISR are studied with MC data samples by varying 

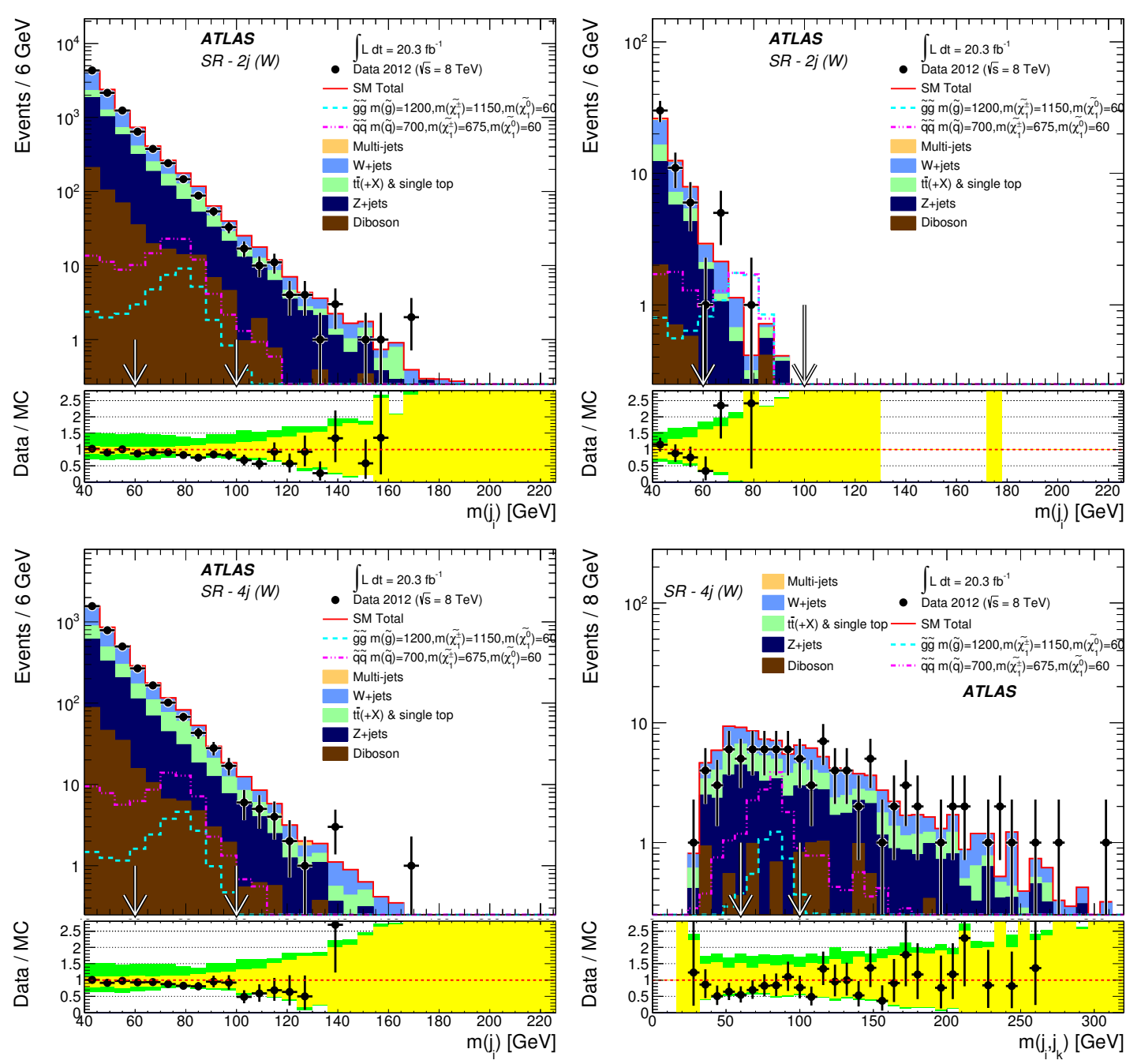

Figure 6. Observed jet and dijet mass distributions for the $2 \mathrm{jW}$ (top) and $4 \mathrm{jW}$ (bottom) signal regions for all unresolved $W$ candidates (left) and for an additional $W$ candidate after requiring at least one unresolved $W$ candidate (right). The additional $W$ candidate is unresolved (SR 2jW, topright) or resolved (SR 4jW, bottom-right). With the exception of the multi-jet background (which is estimated using the data-driven technique described in the text), the histograms denote the MC background expectations prior to the fits described in the text, normalised to cross-section times integrated luminosity. In the lower panels the light (yellow) error bands denote the experimental systematic and MC statistical uncertainties, while the medium dark (green) bands include also the theoretical modelling uncertainty. Expected distributions for benchmark model points are also shown for comparison (masses in $\mathrm{GeV}$ ). Arrows indicate the location of the mass window used in the final selection. See text for discussion of compatibility of data with MC background expectations.

the value of $\alpha_{\mathrm{s}}$, renormalisation and factorisation scales, and the MADGRAPH/PYTHIA matching parameters. For mass splittings $\Delta m<100 \mathrm{GeV}$ the uncertainty ranges from $10 \%$ to $40 \%$ depending on the signal region. For fixed $\Delta m$ the uncertainty is found to be independent of the SUSY particle mass, while for fixed mass it falls approximately exponentially with increasing $\Delta m$, with a characteristic decay constant $\sim 200-300 \mathrm{GeV}$. 


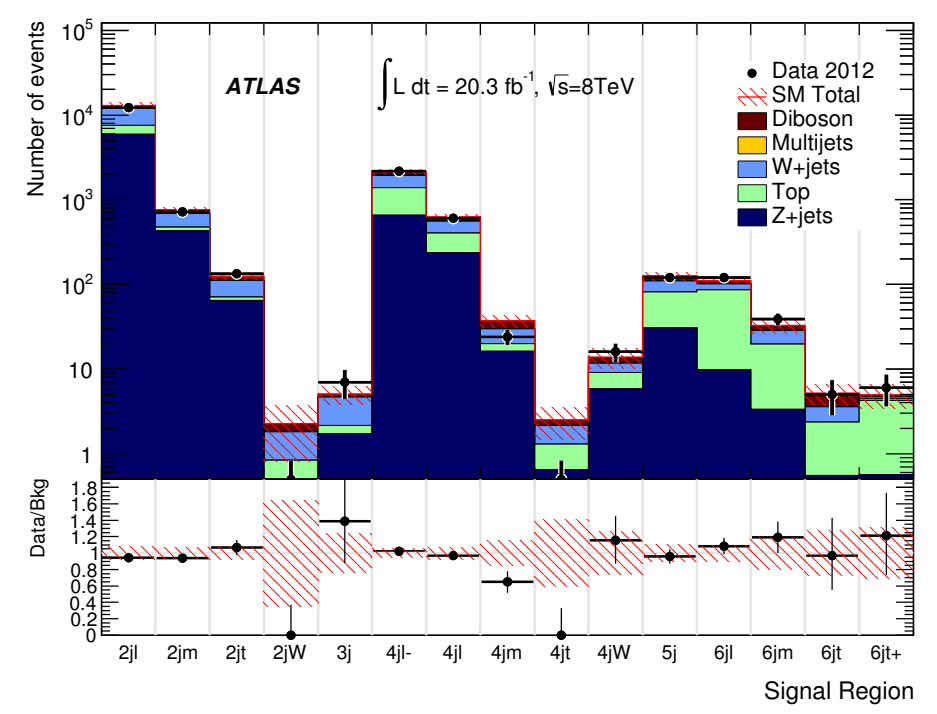

Figure 7. Comparison of the observed and expected event yields as a function of signal region. The background expectations are those obtained from the background-only fits presented in table 5 . In SRs $2 \mathrm{jW}$ and $4 \mathrm{jt}$ no events are observed in the data.

In figure 8 the results are interpreted in the $\tan \beta=30, A_{0}=-2 m_{0}, \mu>0$ slice of mSUGRA/CMSSM [64-69] models. ${ }^{5}$ The mass of the lightest neutral Higgs boson predicted by such models is enhanced relative to that predicted by the mSUGRA/CMSSM models used in previous related ATLAS publications [16-18]. The best-performing signal regions are $6 \mathrm{jt}$ for $m_{0} \gtrsim 1300 \mathrm{GeV}$ and $4 \mathrm{jt}$ for $m_{0} \lesssim 1300 \mathrm{GeV}$, with SR $3 \mathrm{j}$ providing additional sensitivity for $m_{0} \lesssim 400 \mathrm{GeV}$. Results are presented in both the $\left(m_{0}, m_{1 / 2}\right)$ plane and the $\left(m_{\tilde{g}}, m_{\tilde{q}}\right)$-plane. For this model SUSY signal events are generated with HERWIG++-2.5.2. The lower limit on $m_{1 / 2}$ is greater than $380 \mathrm{GeV}$ for $m_{0}<6 \mathrm{TeV}$ and reaches $770 \mathrm{GeV}$ for low values of $m_{0}$. Equal mass light-flavour squarks and gluinos are excluded below $1700 \mathrm{GeV}$ in this scenario.

An interpretation of the results is also presented in figure 9 as a $95 \%$ CL exclusion region in the $\left(m_{\tilde{g}}, m_{\tilde{q}}\right)$-plane for a simplified set of phenomenological MSSM (Minimal Supersymmetric extension of the SM) models $[102,103]$ with $m_{\tilde{\chi}_{1}^{0}}$ equal to $0,395 \mathrm{GeV}$ or $695 \mathrm{GeV}$. In these models the gluino mass and the masses of the 'light'-flavour squarks (of the first two generations, including both $\tilde{q}_{\mathrm{R}}$ and $\tilde{q}_{\mathrm{L}}$, and assuming mass degeneracy) are set to the values shown on the axes of figure 9 . All other supersymmetric particles, including the squarks of the third generation, have their masses set to very high values ('decoupled'). SUSY signal events are generated with MADGRAPH-5.0 interfaced to PYTHIA-6.426. A lower limit of $1650 \mathrm{GeV}$ for equal mass light-flavour squarks and gluinos is found for the scenario with a massless $\tilde{\chi}_{1}^{0}$.

\footnotetext{
${ }^{5}$ Five parameters are needed to specify a particular mSUGRA/CMSSM model: the universal scalar mass, $m_{0}$, the universal gaugino mass $m_{1 / 2}$, the universal trilinear scalar coupling, $A_{0}$, the ratio of the vacuum expectation values of the two Higgs fields, $\tan \beta$, and the sign of the higgsino mass parameter, $\mu= \pm$.
} 

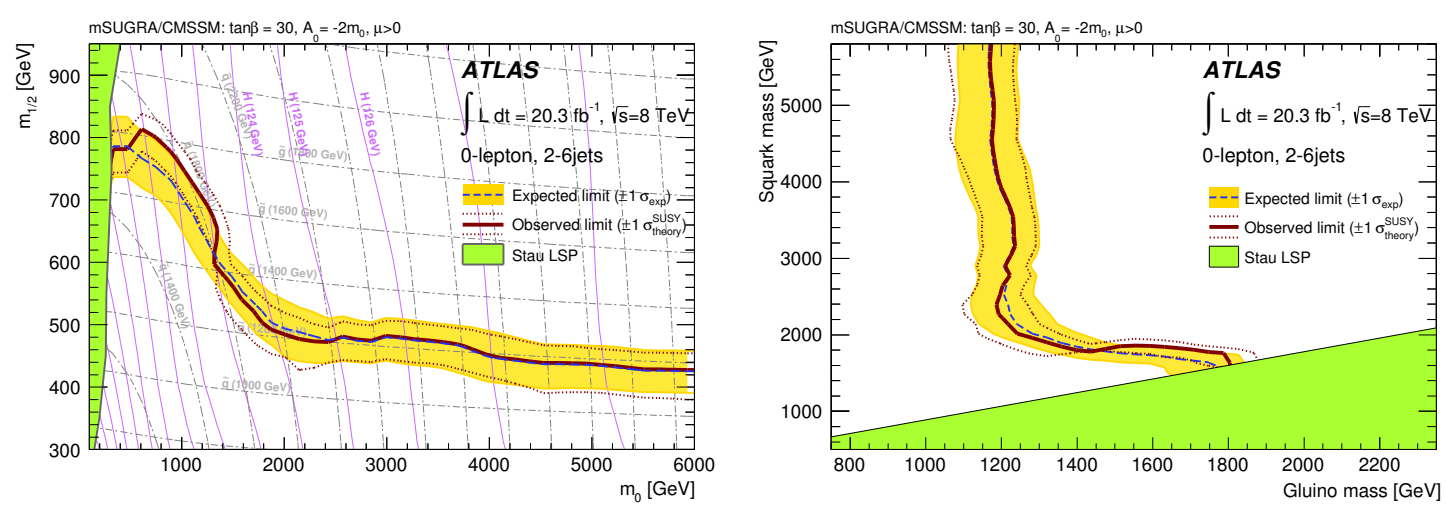

Figure 8. Exclusion limits for mSUGRA/CMSSM models with $\tan \beta=30, A_{0}=-2 m_{0}$ and $\mu>0$ presented (left) in the $\left(m_{0}, m_{1 / 2}\right)$-plane and (right) in the $\left(m_{\tilde{g}}, m_{\tilde{q}}\right)$-plane. Exclusion limits are obtained by using the signal region with the best expected sensitivity at each point. The blue dashed lines show the expected limits at 95\% CL, with the light (yellow) bands indicating the $1 \sigma$ excursions due to experimental and background-only theory uncertainties. Observed limits are indicated by medium dark (maroon) curves, where the solid contour represents the nominal limit, and the dotted lines are obtained by varying the signal cross-section by the renormalisation and factorisation scale and PDF uncertainties.

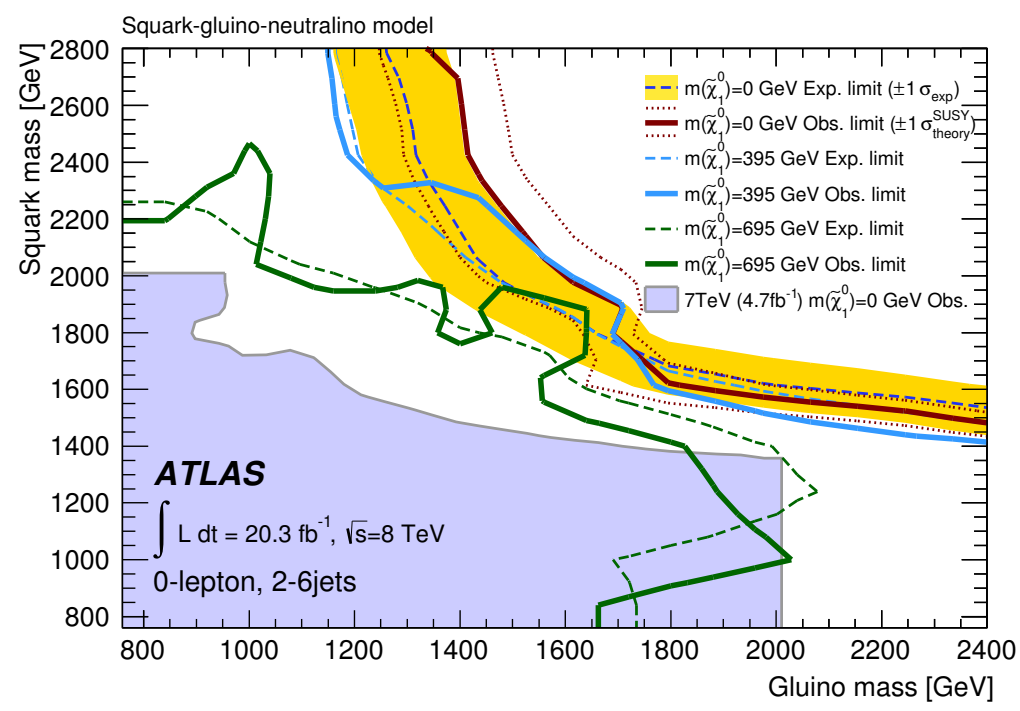

Figure 9. Exclusion limits for a simplified phenomenological MSSM scenario with only strong production of gluinos and first- and second-generation squarks (of common mass), with direct decays to quarks and lightest neutralinos. Three values of the lightest neutralino mass are considered: $m_{\tilde{\chi}_{1}^{0}}=0,395 \mathrm{GeV}$ and $695 \mathrm{GeV}$. Exclusion limits are obtained by using the signal region with the best expected sensitivity at each point. The dashed lines show the expected limits at 95\% CL, with the light (yellow) band indicating the $1 \sigma$ experimental and background-only theory uncertainties on the $m_{\tilde{\chi}_{1}^{0}}=0$ limit. Observed limits are indicated by solid curves. The dotted lines represent the $m_{\tilde{\chi}_{1}^{0}}=0$ observed limits obtained by varying the signal cross-section by the renormalisation and factorisation scale and PDF uncertainties. Previous results for $m_{\tilde{\chi}_{1}^{0}}=0$ from ATLAS at $7 \mathrm{TeV}$ [16] are represented by the shaded (light blue) area. Results at $7 \mathrm{TeV}$ are valid for squark or gluino masses below $2000 \mathrm{GeV}$, the mass range studied for that analysis. 

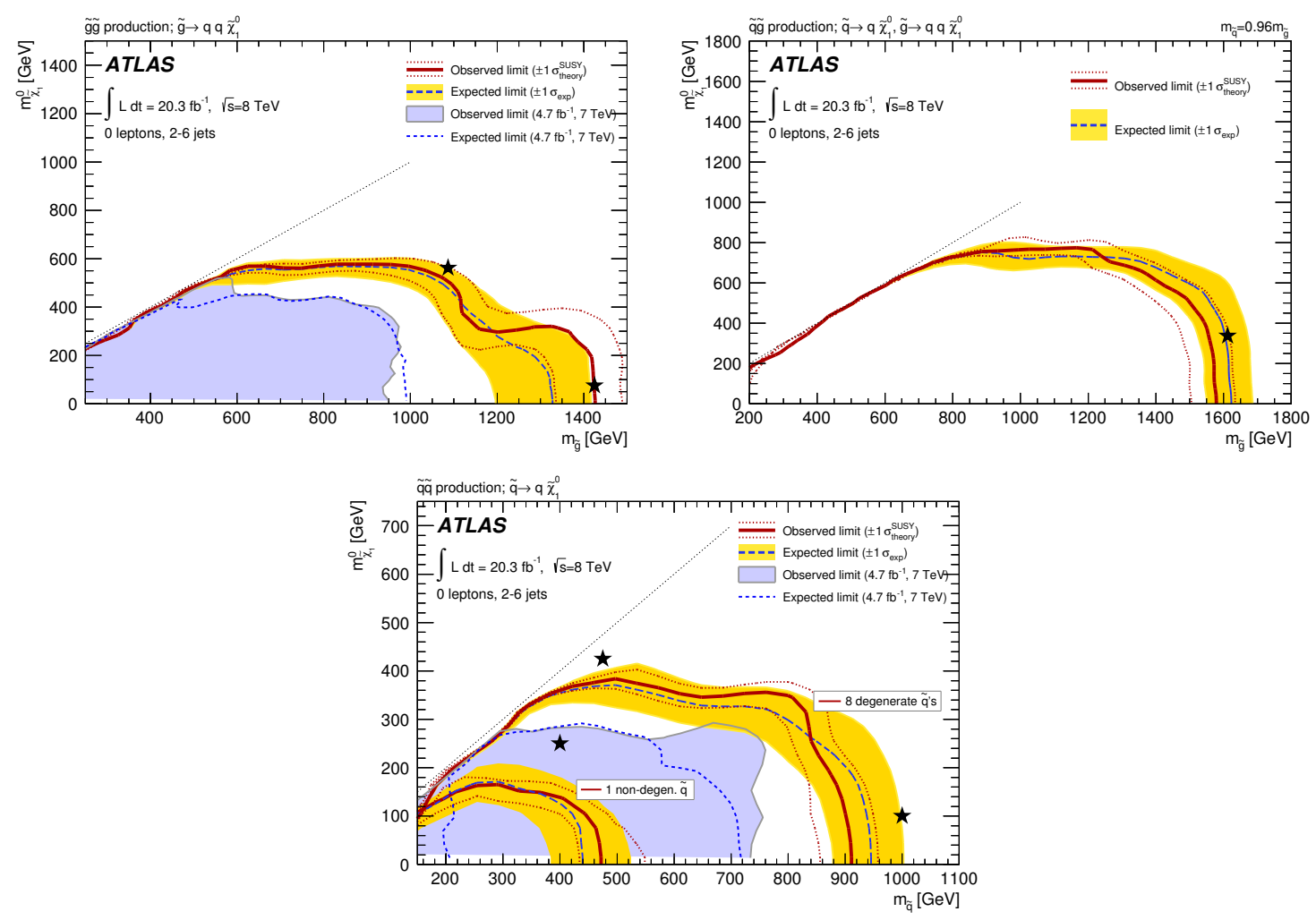

Figure 10. Exclusion limits for direct production of (case (a) - top left) gluino pairs with decoupled squarks, (case (b) - top right) light-flavour squarks and gluinos and (case (c) - bottom) light-flavour squark pairs with decoupled gluinos. Gluinos (light-flavour squarks) are required to decay to two quarks (one quark) and a neutralino LSP. In the bottom figure (case (c)) limits are shown for scenarios with eight degenerate light-flavour squarks $\left(\tilde{q}_{\mathrm{L}}+\tilde{q}_{\mathrm{R}}\right)$, or with only one nondegenerate light-flavour squark produced. Exclusion limits are obtained by using the signal region with the best expected sensitivity at each point. The blue dashed lines show the expected limits at $95 \% \mathrm{CL}$, with the light (yellow) bands indicating the $1 \sigma$ excursions due to experimental and background-only theory uncertainties. Observed limits are indicated by medium dark (maroon) curves, where the solid contour represents the nominal limit, and the dotted lines are obtained by varying the signal cross-section by the renormalisation and factorisation scale and PDF uncertainties. Previous results from ATLAS [16] are represented by the shaded (light blue) areas and light blue dotted lines. The black stars indicate benchmark models used in figures 4-6.

In figure 10 limits are shown for three classes of simplified model in which only direct production of (a) gluino pairs, (b) light-flavour squarks and gluinos or (c) light-flavour squark pairs are considered. All other superpartners, except for the neutralino LSP $\tilde{\chi}_{1}^{0}$, are decoupled thereby forcing each light-flavour squark or gluino to decay directly to one or more quarks and a $\tilde{\chi}_{1}^{0}$. Cross-sections are evaluated assuming decoupled (masses set to $4.5 \mathrm{TeV}$ ) light-flavour squarks or gluinos in cases (a) and (c), respectively. In case (b) the masses of the light-flavour squarks are set to 0.96 times the mass of the gluino, matching the prescription used in refs. [104, 105]. In case (c) limits are shown for scenarios with eight degenerate light-flavour squarks $\left(\tilde{q}_{\mathrm{L}}+\tilde{q}_{\mathrm{R}}\right)$, or with only one non-degenerate light- 

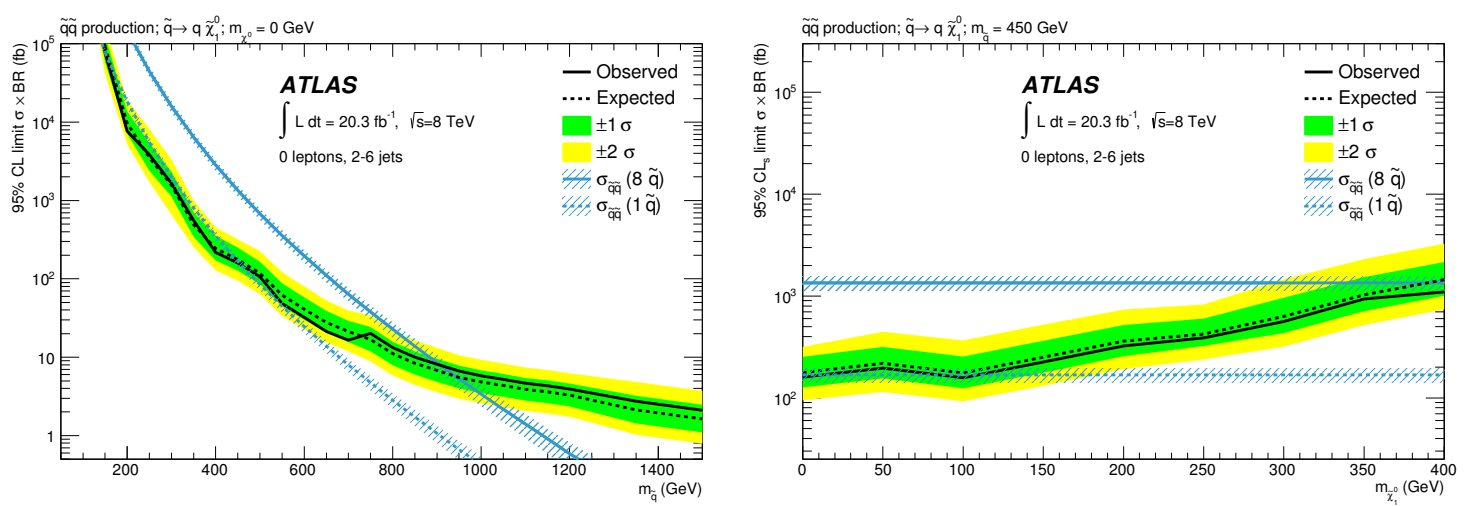

Figure 11. Limits on the light-flavour squark pair production cross-section times branching ratio for models with squark pairs decaying directly into quarks and $\tilde{\chi}_{1}^{0}$ (case (c) in figure 10) as a function of $m_{\tilde{q}}$ for $m_{\tilde{\chi}_{1}^{0}}=0$ (left) and as a function of $m_{\tilde{\chi}_{1}^{0}}$ for $m_{\tilde{q}}=450 \mathrm{GeV}$ (right). Exclusion limits are obtained by using the signal region with the best expected sensitivity at each point. The medium dark (green) band indicates the $1 \sigma$ uncertainty on the expected upper limit, the light (yellow) band the $2 \sigma$ uncertainty. The solid medium dark (blue) line indicates the theoretical inclusive squark pair production cross-section times branching ratio for eight degenerate light-flavour squarks. The dashed medium dark (blue) line indicates the equivalent theoretical cross-section times branching ratio for models in which only one non-degenerate light-flavour squark is produced. The hatched (blue) bands around the theoretical $\sigma$ BR curves denote the scale and PDF uncertainties.

flavour squark produced [106]. For these models SUSY signal events are generated with MADGRAPH-5.0 interfaced to PYTHIA-6.426. Figure 11 presents upper limits for case (c) on the squark pair production cross-section times branching ratio, both as a function of $m_{\tilde{q}}$ for $m_{\tilde{\chi}_{1}^{0}}=0$, and as a function of $m_{\tilde{\chi}_{1}^{0}}$ for $m_{\tilde{q}}=450 \mathrm{GeV}$. In cases (a) and (c), when the $\tilde{\chi}_{1}^{0}$ is massless the lower limit on the gluino mass (case (a)) is $1330 \mathrm{GeV}$, and that on the light-flavour squark mass (case (c)) is $850 \mathrm{GeV}(440 \mathrm{GeV})$ for mass degenerate (single light-flavour) squarks.

In figure 12 limits are shown for pair-produced gluinos each decaying via an intermediate $\tilde{\chi}_{1}^{ \pm}$to two quarks, a $W$ boson and a $\tilde{\chi}_{1}^{0}$, and pair-produced light squarks each decaying via an intermediate $\tilde{\chi}_{1}^{ \pm}$to a quark, a $W$ boson and a $\tilde{\chi}_{1}^{0}$. Results are presented for simplified models in which either the $\tilde{\chi}_{1}^{0}$ mass is fixed to $60 \mathrm{GeV}$, or the mass splitting $(x)$ between the $\tilde{\chi}_{1}^{ \pm}$and the $\tilde{\chi}_{1}^{0}$, relative to that between the squark or gluino and the $\tilde{\chi}_{1}^{0}$, is fixed to $x=0.5$. These models illustrate the sensitivity of this analysis to events with multi-step decay chains involving intermediate $W$ bosons. SUSY signal events are generated with MADGRAPH-5.0 interfaced to PYTHIA-6.426. The lower limit on the gluino (squark) mass extends to $1100 \mathrm{GeV}(700 \mathrm{GeV})$ for a massless $\tilde{\chi}_{1}^{0}$. The use of SRs $2 \mathrm{jW}$ and $4 \mathrm{jW}$ improves sensitivity to models with large $x$, for which the $\tilde{\chi}_{1}^{ \pm}$is nearly degenerate in mass with the squark or gluino. For $x \sim 1.0$ the use of these SRs improves the expected limit on the gluino (squark) mass by approximately $100 \mathrm{GeV}(40 \mathrm{GeV})$.

In figure 13 (left) the results are interpreted in the context of a non-universal Higgs mass model with gaugino mediation (NUHMG) [70] with parameters $m_{0}=0, \tan \beta=10, \mu>0$, $m_{H_{2}}^{2}=0$, and $A_{0}$ chosen to maximise the mass of the lightest Higgs boson. The ranges 

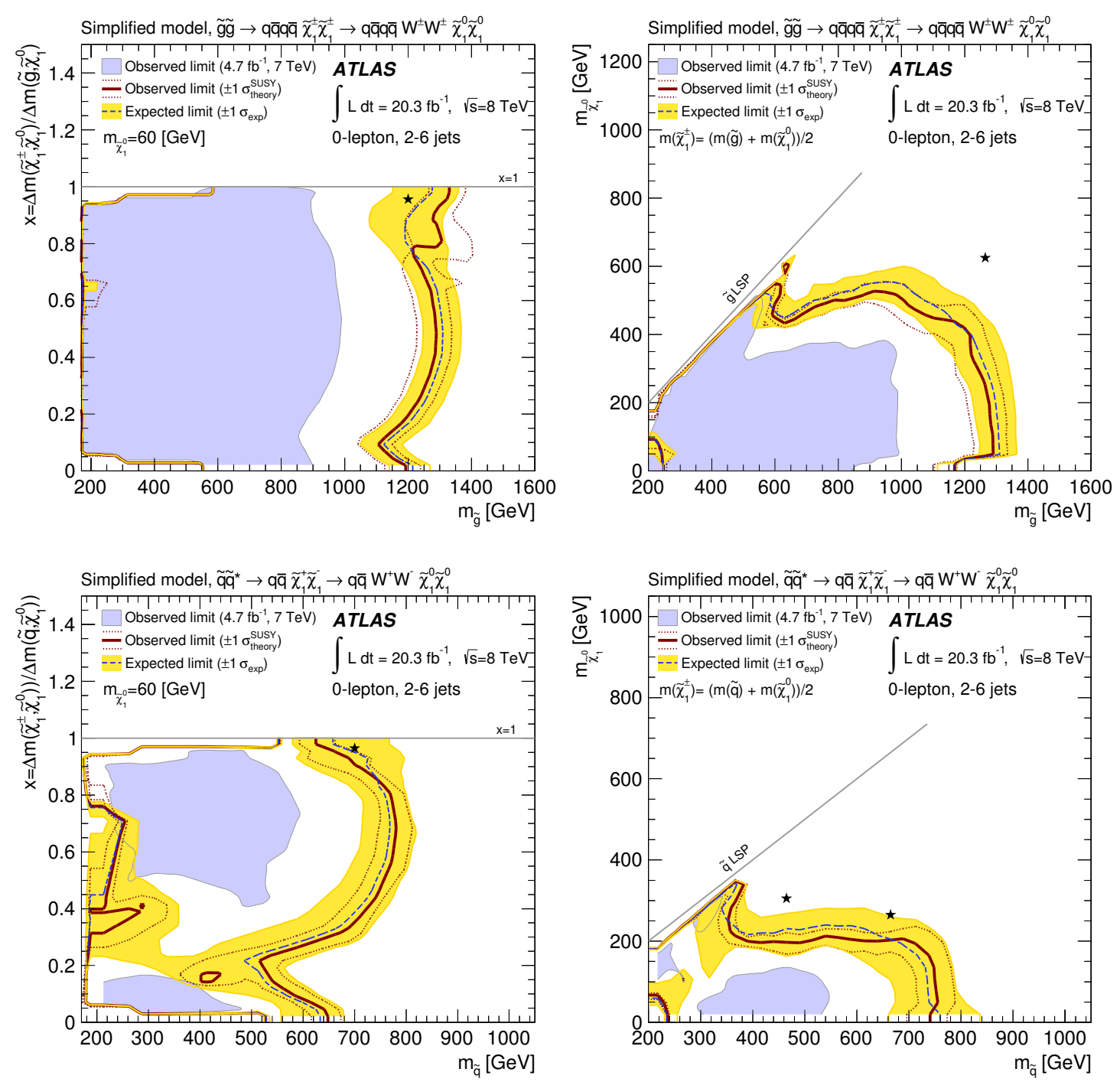

Figure 12. Exclusion limits for pair-produced gluinos each decaying via an intermediate $\tilde{\chi}_{1}^{ \pm}$to two quarks, a $W$ boson and a $\tilde{\chi}_{1}^{0}$ (top) or pair-produced light squarks each decaying via an intermediate $\tilde{\chi}_{1}^{ \pm}$to a quark, a $W$ boson and a $\tilde{\chi}_{1}^{0}$ (bottom). The left-hand figures show results for models with fixed $m\left(\tilde{\chi}_{1}^{0}\right)=60 \mathrm{GeV}$ and varying values of $x=\left(m_{\tilde{\chi}_{1}^{ \pm}}-m_{\tilde{\chi}_{1}^{0}}\right) /\left(m_{y}-m_{\tilde{\chi}_{1}^{0}}\right)$, where $y=\tilde{g}(y=\tilde{q})$ for the top (bottom) figure. The right-hand plots show results for models with a fixed value of $x=1 / 2$ and varying values of $m_{\tilde{\chi}_{1}^{0}}$. Exclusion limits are obtained by using the signal region with the best expected sensitivity at each point. The blue dashed lines show the expected limits at 95\% CL, with the light (yellow) bands indicating the $1 \sigma$ excursions due to experimental and backgroundonly theory uncertainties. Observed limits are indicated by medium dark (maroon) curves, where the solid contour represents the nominal limit, and the dotted lines are obtained by varying the signal cross-section by the renormalisation and factorisation scale and PDF uncertainties. Previous results from ATLAS [16] are represented by the shaded (light blue) areas. The black stars indicate benchmark models used in figures 4-6. 

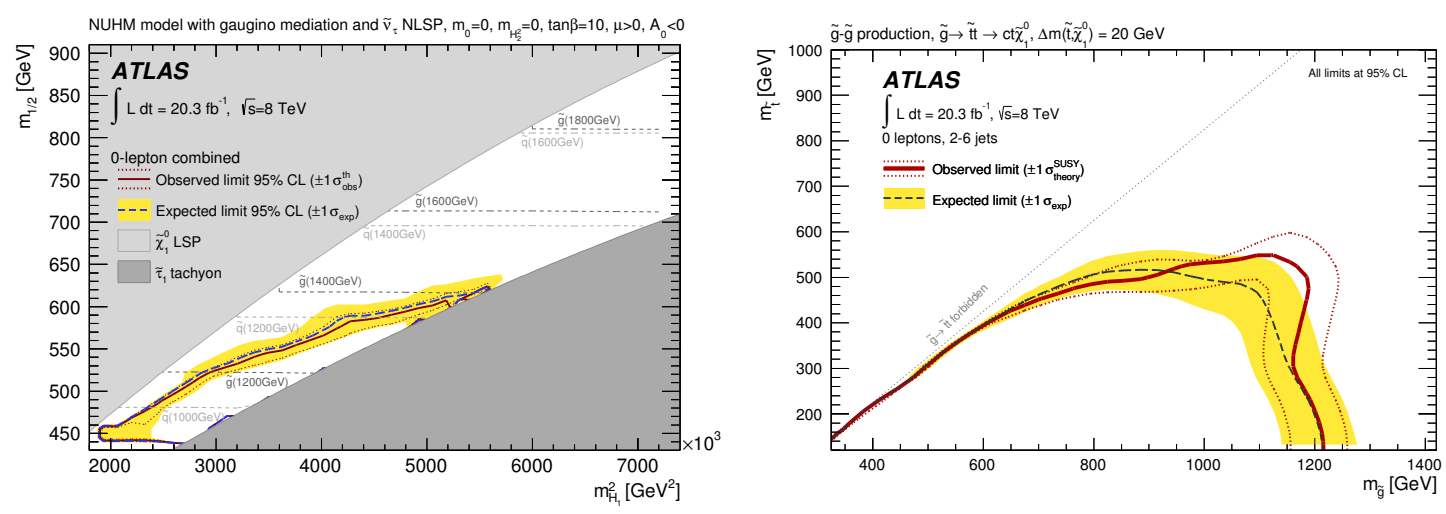

Figure 13. Exclusion limits in the $m_{1 / 2}$ versus $m_{H_{1}}^{2}$ plane for the NUHMG model described in the text (left), and exclusion limits for pair-produced gluinos each decaying into a $\tilde{t}$ and a $\tilde{\chi}_{1}^{0}$, with the subsequent decay $\tilde{t} \rightarrow c \tilde{\chi}_{1}^{0}$ and $\Delta m\left(\tilde{t}, \tilde{\chi}_{1}^{0}\right)=20 \mathrm{GeV}$ (right). Exclusion limits are obtained by using the signal region with the best expected sensitivity at each point. The blue dashed lines show the expected limits at 95\% CL, with the light (yellow) bands indicating the $1 \sigma$ excursions due to experimental and background-only theory uncertainties. Observed limits are indicated by medium dark (maroon) curves, where the solid contour represents the nominal limit, and the dotted lines are obtained by varying the signal cross-section by the renormalisation and factorisation scale and PDF uncertainties. The $m_{\tilde{q}}$ contours in the left-hand figure are calculated using the mean of the masses of the light squarks, excluding those of the top and bottom squarks.

of the two remaining free parameters of the model, $m_{1 / 2}$ and $m_{H_{1}}^{2}$, are chosen such that the next-to-lightest SUSY particle (NLSP) is a tau sneutrino with properties satisfying Big Bang nucleosynthesis constraints. This model is characterised by significant cross-sections for $\tilde{q}$ and $\tilde{g}$ production. SUSY signal events are generated with PYTHIA-6.426.

In figure 13 (right) limits are presented for a simplified phenomenological SUSY model in which pairs of gluinos are produced, each of which then decays to a top squark and a top quark, with the top squark decaying to a charm quark and $\tilde{\chi}_{1}^{0}$. This model is motivated by 'natural' SUSY scenarios with a light top squark and a small mass splitting between the top squark and the $\tilde{\chi}_{1}^{0}$ leading to co-annihilation between top squarks and $\tilde{\chi}_{1}^{0}$ dark matter particles in the early universe. SUSY signal events are generated with HERWIG++-2.5.2. The lower limit on the gluino mass extends to $1110 \mathrm{GeV}$ for a top squark of mass $400 \mathrm{GeV}$.

\section{Conclusions}

This paper reports a search for squarks and gluinos in final states containing high- $p_{\mathrm{T}}$ jets, large missing transverse momentum and no electrons or muons, based on a $20.3 \mathrm{fb}^{-1}$ dataset of $\sqrt{s}=8 \mathrm{TeV}$ proton-proton collisions recorded by the ATLAS experiment at the LHC in 2012. Good agreement is seen between the numbers of events observed in the data and the numbers of events expected from SM processes.

Results are interpreted in terms of mSUGRA/CMSSM models with $\tan \beta=30, A_{0}=$ $-2 m_{0}$ and $\mu>0$, and in terms of simplified models with only light-flavour squarks, or gluinos, or both, together with a neutralino LSP, with the other SUSY particles decou- 
pled. The results are also interpreted in terms of several other SUSY models. In the mSUGRA/CMSSM models, the $95 \%$ confidence level exclusion limit on $m_{1 / 2}$ is greater than $380 \mathrm{GeV}$ for $m_{0}<6 \mathrm{TeV}$ and reaches $770 \mathrm{GeV}$ for low $m_{0}$. Equal mass squarks and gluinos are excluded below $1700 \mathrm{GeV}$ in this scenario. A lower limit of $1650 \mathrm{GeV}$ for equal mass light-flavour squarks and gluinos is found for simplified MSSM models with a massless lightest neutralino. For a massless lightest neutralino, gluino masses below $1330 \mathrm{GeV}$ are excluded at the $95 \%$ confidence level in a simplified model with only gluinos and the lightest neutralino. For a simplified model involving the strong production of squarks of the first and second generations, with decays to a massless lightest neutralino, squark masses below $850 \mathrm{GeV}(440 \mathrm{GeV})$ are excluded, assuming mass degenerate (single light-flavour) squarks. For simplified models involving the pair production of gluinos, each decaying to a top squark and a top quark, with the top squark decaying to a charm quark and a neutralino, the lower limit on the gluino mass extends to $1110 \mathrm{GeV}$ for a top squark of mass $400 \mathrm{GeV}$. These results extend the region of supersymmetric parameter space excluded by previous searches with the ATLAS detector.

\section{Acknowledgments}

We thank CERN for the very successful operation of the LHC, as well as the support staff from our institutions without whom ATLAS could not be operated efficiently.

We acknowledge the support of ANPCyT, Argentina; YerPhI, Armenia; ARC, Australia; BMWF and FWF, Austria; ANAS, Azerbaijan; SSTC, Belarus; CNPq and FAPESP, Brazil; NSERC, NRC and CFI, Canada; CERN; CONICYT, Chile; CAS, MOST and NSFC, China; COLCIENCIAS, Colombia; MSMT CR, MPO CR and VSC CR, Czech Republic; DNRF, DNSRC and Lundbeck Foundation, Denmark; EPLANET, ERC and NSRF, European Union; IN2P3-CNRS, CEA-DSM/IRFU, France; GNSF, Georgia; BMBF, DFG, HGF, MPG and AvH Foundation, Germany; GSRT and NSRF, Greece; ISF, MINERVA, GIF, I-CORE and Benoziyo Center, Israel; INFN, Italy; MEXT and JSPS, Japan; CNRST, Morocco; FOM and NWO, Netherlands; BRF and RCN, Norway; MNiSW and NCN, Poland; GRICES and FCT, Portugal; MNE/IFA, Romania; MES of Russia and ROSATOM, Russian Federation; JINR; MSTD, Serbia; MSSR, Slovakia; ARRS and MIZŠ, Slovenia; DST/NRF, South Africa; MINECO, Spain; SRC and Wallenberg Foundation, Sweden; SER, SNSF and Cantons of Bern and Geneva, Switzerland; NSC, Taiwan; TAEK, Turkey; STFC, the Royal Society and Leverhulme Trust, United Kingdom; DOE and NSF, United States of America.

The crucial computing support from all WLCG partners is acknowledged gratefully, in particular from CERN and the ATLAS Tier-1 facilities at TRIUMF (Canada), NDGF (Denmark, Norway, Sweden), CC-IN2P3 (France), KIT/GridKA (Germany), INFN-CNAF (Italy), NL-T1 (Netherlands), PIC (Spain), ASGC (Taiwan), RAL (U.K.) and BNL (U.S.A.) and in the Tier-2 facilities worldwide.

Open Access. This article is distributed under the terms of the Creative Commons Attribution License (CC-BY 4.0), which permits any use, distribution and reproduction in any medium, provided the original author(s) and source are credited. 


\section{References}

[1] L. Evans and P. Bryant, LHC Machine, 2008 JINST 3 S08001 [INSPIRE].

[2] H. Miyazawa, Baryon Number Changing Currents, Prog. Theor. Phys. 36 (1966) 1266.

[3] P. Ramond, Dual Theory for Free Fermions, Phys. Rev. D 3 (1971) 2415 [InSPIRE].

[4] Y. Golfand and E.P. Likhtman, Extension of the Algebra of Poincaré Group Generators and Violation of p Invariance, JETP Lett. 13 (1971) 323 [Pisma Zh. Eksp. Teor. Fiz. 13 (1971) 452] [INSPIRE].

[5] A. Neveu and J.H. Schwarz, Factorizable dual model of pions, Nucl. Phys. B 31 (1971) 86 [INSPIRE].

[6] A. Neveu and J.H. Schwarz, Quark Model of Dual Pions, Phys. Rev. D 4 (1971) 1109 [INSPIRE].

[7] J.-L. Gervais and B. Sakita, Field Theory Interpretation of Supergauges in Dual Models, Nucl. Phys. B 34 (1971) 632 [inSPIRE].

[8] D.V. Volkov and V.P. Akulov, Is the Neutrino a Goldstone Particle?, Phys. Lett. B 46 (1973) 109 [inSPIRE].

[9] J. Wess and B. Zumino, A Lagrangian Model Invariant Under Supergauge Transformations, Phys. Lett. B 49 (1974) 52 [InSPIRE].

[10] J. Wess and B. Zumino, Supergauge Transformations in Four-Dimensions, Nucl. Phys. B 70 (1974) 39 [InSPIRE].

[11] P. Fayet, Supersymmetry and Weak, Electromagnetic and Strong Interactions, Phys. Lett. B 64 (1976) 159 [INSPIRE].

[12] P. Fayet, Spontaneously Broken Supersymmetric Theories of Weak, Electromagnetic and Strong Interactions, Phys. Lett. B 69 (1977) 489 [INSPIRE].

[13] G.R. Farrar and P. Fayet, Phenomenology of the Production, Decay and Detection of New Hadronic States Associated with Supersymmetry, Phys. Lett. B 76 (1978) 575 [INSPIRE].

[14] P. Fayet, Relations Between the Masses of the Superpartners of Leptons and Quarks, the Goldstino Couplings and the Neutral Currents, Phys. Lett. B 84 (1979) 416 [INSPIRE].

[15] S. Dimopoulos and H. Georgi, Softly Broken Supersymmetry and SU(5), Nucl. Phys. B 193 (1981) 150 [INSPIRE].

[16] ATLAS collaboration, Search for squarks and gluinos with the ATLAS detector in final states with jets and missing transverse momentum using $4.7 \mathrm{fb}^{-1}$ of $\sqrt{s}=7 \mathrm{TeV}$ proton-proton collision data, Phys. Rev. D 87 (2013) 012008 [arXiv:1208.0949] [INSPIRE].

[17] ATLAS collaboration, Search for squarks and gluinos using final states with jets and missing transverse momentum with the ATLAS detector in $\sqrt{s}=7$ TeV proton-proton collisions, Phys. Lett. B 710 (2012) 67 [arXiv:1109.6572] [INSPIRE].

[18] ATLAS collaboration, Search for squarks and gluinos using final states with jets and missing transverse momentum with the ATLAS detector in $\sqrt{s}=7 \mathrm{TeV}$ proton-proton collisions, Phys. Lett. B 701 (2011) 186 [arXiv:1102.5290] [INSPIRE].

[19] CMS collaboration, Search for supersymmetry in hadronic final states with missing transverse energy using the variables $\alpha_{T}$ and b-quark multiplicity in pp collisions at $\sqrt{s}=8 \mathrm{TeV}$, Eur. Phys. J. C 73 (2013) 2568 [arXiv:1303.2985] [INSPIRE]. 
[20] CMS collaboration, Inclusive search for supersymmetry using the razor variables in $p p$ collisions at $\sqrt{s}=7 \mathrm{TeV}$, Phys. Rev. Lett. 111 (2013) 081802 [arXiv:1212.6961] [InSPIRE].

[21] CMS collaboration, Search for supersymmetry in hadronic final states using MT2 in pp collisions at $\sqrt{s}=7 \mathrm{TeV}$, JHEP 10 (2012) 018 [arXiv:1207.1798] [INSPIRE].

[22] CMS collaboration, Search for new physics in the multijet and missing transverse momentum final state in proton-proton collisions at $\sqrt{s}=7 \mathrm{TeV}$,

Phys. Rev. Lett. 109 (2012) 171803 [arXiv:1207.1898] [inSPIRE].

[23] ATLAS collaboration, Further search for supersymmetry at $\sqrt{s}=7 \mathrm{TeV}$ in final states with jets, missing transverse momentum and isolated leptons with the ATLAS detector, Phys. Rev. D 86 (2012) 092002 [arXiv:1208.4688] [InSPIRE].

[24] ATLAS collaboration, The ATLAS Experiment at the CERN Large Hadron Collider, 2008 JINST 3 S08003 [INSPIRE].

[25] ATLAS collaboration, Improved luminosity determination in pp collisions at $\sqrt{s}=7 \mathrm{TeV}$ using the ATLAS detector at the LHC, Eur. Phys. J. C 73 (2013) 2518 [arXiv:1302.4393] [INSPIRE].

[26] ATLAS collaboration, Measurements of top quark pair relative differential cross-sections with ATLAS in pp collisions at $\sqrt{s}=7 \mathrm{TeV}$, Eur. Phys. J. C 73 (2013) 2261 [arXiv:1207.5644] [INSPIRE].

[27] ATLAS collaboration, Measurements of normalized differential cross-sections for $t \bar{t}$ production in pp collisions at $\sqrt{s}=7 \mathrm{TeV}$ using the ATLAS detector, arXiv:1407.0371 [INSPIRE].

[28] T. Gleisberg et al., Event generation with SHERPA 1.1, JHEP 02 (2009) 007 [arXiv:0811.4622] [INSPIRE].

[29] S. Catani, L. Cieri, G. Ferrera, D. de Florian and M. Grazzini, Vector boson production at hadron colliders: a fully exclusive QCD calculation at $N N L O$,

Phys. Rev. Lett. 103 (2009) 082001 [arXiv:0903.2120] [INSPIRE].

[30] H.-L. Lai et al., New parton distributions for collider physics, Phys. Rev. D 82 (2010) 074024 [arXiv: 1007.2241] [INSPIRE].

[31] M.L. Mangano, M. Moretti, F. Piccinini, R. Pittau and A.D. Polosa, ALPGEN, a generator for hard multiparton processes in hadronic collisions, JHEP 07 (2003) 001 [hep-ph/0206293] [INSPIRE].

[32] ATLAS collaboration, First tuning of HERWIG/JIMMY to ATLAS data, ATL-PHYS-PUB-2010-014 (2010).

[33] J. Pumplin et al., New generation of parton distributions with uncertainties from global QCD analysis, JHEP 07 (2002) 012 [hep-ph/0201195] [INSPIRE].

[34] G. Corcella et al., HERWIG 6: An Event generator for hadron emission reactions with interfering gluons (including supersymmetric processes), JHEP 01 (2001) 010 [hep-ph/0011363] [INSPIRE].

[35] G. Corcella et al., HERWIG 6.5 release note, hep-ph/0210213 [INSPIRE].

[36] P. Nason, A new method for combining NLO QCD with shower Monte Carlo algorithms, JHEP 11 (2004) 040 [hep-ph/0409146] [INSPIRE]. 
[37] S. Frixione, P. Nason and C. Oleari, Matching NLO QCD computations with Parton Shower simulations: the POWHEG method, JHEP 11 (2007) 070 [arXiv: 0709. 2092] [INSPIRE].

[38] S. Alioli, P. Nason, C. Oleari and E. Re, A general framework for implementing NLO calculations in shower Monte Carlo programs: the POWHEG BOX, JHEP 06 (2010) 043 [arXiv: 1002.2581] [INSPIRE].

[39] M. Czakon, P. Fiedler and A. Mitov, Total Top-Quark Pair-Production Cross Section at Hadron Colliders Through $O\left(\alpha_{S}^{4}\right)$, Phys. Rev. Lett. 110 (2013) 252004 [arXiv:1303.6254] [INSPIRE].

[40] M. Czakon and A. Mitov, Top++: A Program for the Calculation of the Top-Pair Cross-Section at Hadron Colliders, Comput. Phys. Commun. 185 (2014) 2930 [arXiv:1112.5675] [INSPIRE].

[41] T. Sjöstrand, S. Mrenna and P.Z. Skands, PYTHIA 6.4 Physics and Manual, JHEP 05 (2006) 026 [hep-ph/0603175] [INSPIRE].

[42] B. Cooper et al., Importance of a consistent choice of $\alpha_{s}$ in the matching of ALPGEN and PYTHIA, Eur. Phys. J. C 72 (2012) 2078 [arXiv:1109.5295] [inSPIRE].

[43] P.Z. Skands, Tuning Monte Carlo Generators: The Perugia Tunes, Phys. Rev. D 82 (2010) 074018 [arXiv:1005.3457] [InSPIRE].

[44] S. Frixione and B.R. Webber, Matching NLO QCD computations and parton shower simulations, JHEP 06 (2002) 029 [hep-ph/0204244] [INSPIRE].

[45] S. Frixione, P. Nason and B.R. Webber, Matching NLO QCD and parton showers in heavy flavor production, JHEP 08 (2003) 007 [hep-ph/0305252] [INSPIRE].

[46] B.P. Kersevan and E. Richter-Was, The Monte Carlo event generator AcerMC versions 2.0 to 3.8 with interfaces to PYTHIA 6.4, HERWIG 6.5 and ARIADNE 4.1, Comput. Phys. Commun. 184 (2013) 919 [hep-ph/0405247] [INSPIRE].

[47] N. Kidonakis, Next-to-next-to-leading-order collinear and soft gluon corrections for t-channel single top quark production, Phys. Rev. D 83 (2011) 091503 [arXiv:1103.2792] [INSPIRE].

[48] N. Kidonakis, NNLL resummation for s-channel single top quark production, Phys. Rev. D 81 (2010) 054028 [arXiv:1001.5034] [INSPIRE].

[49] N. Kidonakis, Two-loop soft anomalous dimensions for single top quark associated production with a W- or H-, Phys. Rev. D 82 (2010) 054018 [arXiv:1005.4451] [INSPIRE].

[50] J. Alwall, M. Herquet, F. Maltoni, O. Mattelaer and T. Stelzer, MadGraph 5: Going Beyond, JHEP 06 (2011) 128 [arXiv:1106.0522] [INSPIRE].

[51] A. Lazopoulos, T. McElmurry, K. Melnikov and F. Petriello, Next-to-leading order QCD corrections to $t \bar{t} Z$ production at the LHC, Phys. Lett. B 666 (2008) 62 [arXiv:0804.2220] [INSPIRE].

[52] M.V. Garzelli, A. Kardos, C.G. Papadopoulos and Z. Trócsányi, $t \bar{t} W^{+-}$and $t \bar{t} Z$ Hadroproduction at NLO accuracy in QCD with Parton Shower and Hadronization effects, JHEP 11 (2012) 056 [arXiv:1208.2665] [INSPIRE].

[53] J.M. Campbell and R.K. Ellis, $t \bar{t} W^{ \pm}$production and decay at NLO, JHEP 07 (2012) 052 [arXiv: 1204.5678] [INSPIRE].

[54] J.M. Campbell and R.K. Ellis, An Update on vector boson pair production at hadron colliders, Phys. Rev. D 60 (1999) 113006 [hep-ph/9905386] [INSPIRE]. 
[55] J.M. Campbell, R.K. Ellis and C. Williams, Vector boson pair production at the LHC, JHEP 07 (2011) 018 [arXiv:1105.0020] [INSPIRE].

[56] M. Bähr et al., HERWIG++ Physics and Manual, Eur. Phys. J. C 58 (2008) 639 [arXiv:0803.0883] [INSPIRE].

[57] M.L. Mangano, M. Moretti, F. Piccinini and M. Treccani, Matching matrix elements and shower evolution for top-quark production in hadronic collisions, JHEP 01 (2007) 013 [hep-ph/0611129] [INSPIRE].

[58] W. Beenakker, R. Hopker, M. Spira and P.M. Zerwas, Squark and gluino production at hadron colliders, Nucl. Phys. B 492 (1997) 51 [hep-ph/9610490] [InSPIRE].

[59] A. Kulesza and L. Motyka, Threshold resummation for squark-antisquark and gluino-pair production at the LHC, Phys. Rev. Lett. 102 (2009) 111802 [arXiv:0807.2405] [INSPIRE].

[60] A. Kulesza and L. Motyka, Soft gluon resummation for the production of gluino-gluino and squark-antisquark pairs at the LHC, Phys. Rev. D 80 (2009) 095004 [arXiv: 0905.4749] [INSPIRE].

[61] W. Beenakker et al., Soft-gluon resummation for squark and gluino hadroproduction, JHEP 12 (2009) 041 [arXiv:0909.4418] [INSPIRE].

[62] W. Beenakker et al., Squark and gluino hadroproduction, Int. J. Mod. Phys. A 26 (2011) 2637 [arXiv:1105.1110] [InSPIRE].

[63] M. Krämer et al., Supersymmetry production cross sections in pp collisions at $\sqrt{s}=7 \mathrm{TeV}$, arXiv:1206.2892 [INSPIRE].

[64] A.H. Chamseddine, R.L. Arnowitt and P. Nath, Locally Supersymmetric Grand Unification, Phys. Rev. Lett. 49 (1982) 970 [INSPIRE].

[65] R. Barbieri, S. Ferrara and C.A. Savoy, Gauge Models with Spontaneously Broken Local Supersymmetry, Phys. Lett. B 119 (1982) 343 [INSPIRE].

[66] L.E. Ibáñez, Locally Supersymmetric SU(5) Grand Unification, Phys. Lett. B 118 (1982) 73 [INSPIRE].

[67] L.J. Hall, J.D. Lykken and S. Weinberg, Supergravity as the Messenger of Supersymmetry Breaking, Phys. Rev. D 27 (1983) 2359 [InSPIRE].

[68] N. Ohta, Grand unified theories based on local supersymmetry, Prog. Theor. Phys. 70 (1983) 542 [INSPIRE].

[69] G.L. Kane, C.F. Kolda, L. Roszkowski and J.D. Wells, Study of constrained minimal supersymmetry, Phys. Rev. D 49 (1994) 6173 [hep-ph/9312272] [INSPIRE].

[70] L. Covi and S. Kraml, Collider signatures of gravitino dark matter with a sneutrino NLSP, JHEP 08 (2007) 015 [hep-ph/0703130] [INSPIRE].

[71] A. Djouadi, M.M. Muhlleitner and M. Spira, Decays of supersymmetric particles: The Program SUSY-HIT (SUspect-SdecaY-HDECAY-InTerface), Acta Phys. Polon. B 38 (2007) 635 [hep-ph/0609292] [INSPIRE].

[72] B.C. Allanach, SOFTSUSY: a program for calculating supersymmetric spectra, Comput. Phys. Commun. 143 (2002) 305 [hep-ph/0104145] [INSPIRE].

[73] M. Muhlleitner, A. Djouadi and Y. Mambrini, SDECAY: A Fortran code for the decays of the supersymmetric particles in the MSSM, Comput. Phys. Commun. 168 (2005) 46 [hep-ph/0311167] [INSPIRE]. 
[74] ATLAS collaboration, ATLAS tunes of PYTHIA 6 and PYTHIA 8 for MC11, ATL-PHYS-PUB-2011-009 (2011).

[75] ATLAS collaboration, Further ATLAS tunes of PYTHIA6 and PYTHIA 8, ATL-PHYS-PUB-2011-014 (2011).

[76] ATLAS collaboration, First tuning of HERWIG/JIMMY to ATLAS data, ATL-PHYS-PUB-2010-014 (2010).

[77] ATLAS collaboration, The ATLAS Simulation Infrastructure, Eur. Phys. J. C 70 (2010) 823 [arXiv: 1005.4568] [InSPIRE].

[78] GEANT4 collaboration, S. Agostinelli et al., GEANT4: A Simulation toolkit, Nucl. Instrum. Meth. A 506 (2003) 250 [INSPIRE].

[79] ATLAS collaboration, The simulation principle and performance of the ATLAS fast calorimeter simulation FastCaloSim, ATL-PHYS-PUB-2010-013 (2010).

[80] M. Cacciari, G.P. Salam and G. Soyez, The anti- $k_{t}$ jet clustering algorithm, JHEP 04 (2008) 063 [arXiv:0802.1189] [INSPIRE].

[81] M. Cacciari and G.P. Salam, Dispelling the $N^{3}$ myth for the $k_{t}$ jet-finder, Phys. Lett. B 641 (2006) 57 [hep-ph/0512210] [INSPIRE].

[82] ATLAS collaboration, Jet energy measurement with the ATLAS detector in proton-proton collisions at $\sqrt{s}=7 \mathrm{TeV}$, Eur. Phys. J. C 73 (2013) 2304 [arXiv:1112.6426] [INSPIRE].

[83] W. Lampl et al., Calorimeter Clustering Algorithms: Description and Performance, ATL-LARG-PUB-2008-002 (2008).

[84] M. Cacciari and G.P. Salam, Pileup subtraction using jet areas, Phys. Lett. B 659 (2008) 119 [arXiv:0707.1378] [INSPIRE].

[85] ATLAS collaboration, Pile-up subtraction and suppression for jets in ATLAS, ATLAS-CONF-2013-083 (2013).

[86] C. Issever, K. Borras and D. Wegener, An Improved weighting algorithm to achieve software compensation in a fine grained LAr calorimeter, Nucl. Instrum. Meth. A 545 (2005) 803 [physics/0408129] [INSPIRE].

[87] ATLAS collaboration, Jet energy scale and its systematic uncertainty in proton-proton collisions at $\sqrt{s}=7$ TeV with ATLAS 2011 data, ATLAS-CONF-2013-004 (2013).

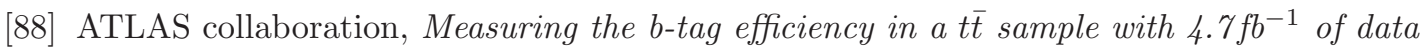
from the ATLAS detector, ATLAS-CONF-2012-097 (2012).

[89] ATLAS collaboration, Electron reconstruction and identification efficiency measurements with the ATLAS detector using the 2011 LHC proton-proton collision data, Eur. Phys. J. C 74 (2014) 2941 [arXiv: 1404.2240] [INSPIRE].

[90] ATLAS collaboration, Muon reconstruction efficiency and momentum resolution of the ATLAS experiment in proton-proton collisions at $\sqrt{s}=7 \mathrm{TeV}$ in 2010, Eur. Phys. J. C 74 (2014) 3034 [arXiv: 1404.4562] [INSPIRE].

[91] ATLAS collaboration, Performance of Missing Transverse Momentum Reconstruction in Proton-Proton Collisions at 7TeV with ATLAS, Eur. Phys. J. C 72 (2012) 1844 [arXiv: 1108.5602] [INSPIRE]. 
[92] ATLAS collaboration, Performance of Missing Transverse Momentum Reconstruction in ATLAS studied in Proton-Proton Collisions recorded in 2012 at $\sqrt{s}=8 \mathrm{TeV}$,

ATLAS-CONF-2013-082 (2013).

[93] ATLAS collaboration, Measurements of the photon identification efficiency with the ATLAS detector using $4.9 \mathrm{fb}^{-1}$ of pp collision data collected in 2011, ATLAS-CONF-2012-123 (2012).

[94] ATLAS collaboration, Performance of the Reconstruction and Identification of Hadronic $\tau$ Decays in ATLAS with 2011 Data, ATLAS-CONF-2012-142 (2012).

[95] ATLAS collaboration, Characterisation and mitigation of beam-induced backgrounds observed in the ATLAS detector during the 2011 proton-proton run, 2013 JINST 8 P07004 [arXiv: 1303.0223] [INSPIRE].

[96] ATLAS collaboration, Selection of jets produced in proton-proton collisions with the ATLAS detector using 2011 data, ATLAS-CONF-2012-020 (2012).

[97] ATLAS collaboration, Measurement of the $Z / \gamma^{*}$ boson transverse momentum distribution in pp collisions at $\sqrt{s}=7 \mathrm{TeV}$ with the ATLAS detector, arXiv:1406.3660 [INSPIRE].

[98] G. Cowan, K. Cranmer, E. Gross and O. Vitells, Asymptotic formulae for likelihood-based tests of new physics, Eur. Phys. J. C 71 (2011) 1554 [arXiv:1007.1727] [InSPIRE].

[99] ATLAS collaboration, Single hadron response measurement and calorimeter jet energy scale uncertainty with the ATLAS detector at the LHC, Eur. Phys. J. C 73 (2013) 2305 [arXiv: 1203.1302] [INSPIRE].

[100] ATLAS collaboration, Jet energy resolution in proton-proton collisions at $\sqrt{s}=7 \mathrm{TeV}$ recorded in 2010 with the ATLAS detector, Eur. Phys. J. C 73 (2013) 2306 [arXiv:1210.6210] [INSPIRE].

[101] A.L. Read, Presentation of search results: the CL(s) technique, J. Phys. G 28 (2002) 2693 [INSPIRE].

[102] J. Alwall, P. Schuster and N. Toro, Simplified Models for a First Characterization of New Physics at the LHC, Phys. Rev. D 79 (2009) 075020 [arXiv: 0810. 3921] [InSPIRE].

[103] LHC New Physics Working Group collaboration, D. Alves et al., Simplified Models for LHC New Physics Searches, J. Phys. G 39 (2012) 105005 [arXiv:1105.2838] [InSPIRE].

[104] T.J. LeCompte and S.P. Martin, Large Hadron Collider reach for supersymmetric models with compressed mass spectra, Phys. Rev. D 84 (2011) 015004 [arXiv:1105.4304] [INSPIRE].

[105] T.J. LeCompte and S.P. Martin, Compressed supersymmetry after 1/fb at the Large Hadron Collider, Phys. Rev. D 85 (2012) 035023 [arXiv:1111.6897] [InSPIRE].

[106] R. Mahbubani, M. Papucci, G. Perez, J.T. Ruderman and A. Weiler, Light Nondegenerate Squarks at the LHC, Phys. Rev. Lett. 110 (2013) 151804 [arXiv:1212.3328] [InSPIRE]. 


\section{The ATLAS collaboration}

G. $\operatorname{Aad}^{84}$, B. Abbott ${ }^{112}$, J. Abdallah ${ }^{152}$, S. Abdel Khalek ${ }^{116}$, O. Abdinov ${ }^{11}$, R. Aben ${ }^{106}$, B. Abi ${ }^{113}$, M. Abolins ${ }^{89}$, O.S. AbouZeid ${ }^{159}$, H. Abramowicz ${ }^{154}$, H. Abreu ${ }^{153}$, R. Abreu ${ }^{30}$, Y. Abulaiti 147a,147b, B.S. Acharya ${ }^{165 a, 165 b, a}$, L. Adamczyk ${ }^{38 a}$, D.L. Adams ${ }^{25}$, J. Adelman ${ }^{177}$, S. Adomeit ${ }^{99}$, T. Adye ${ }^{130}$, T. Agatonovic-Jovin ${ }^{13 a}$, J.A. Aguilar-Saavedra ${ }^{125 a, 125 f}$, M. Agustoni ${ }^{17}$, S.P. Ahlen ${ }^{22}$, F. Ahmadov ${ }^{64, b}$, G. Aielli ${ }^{134 a, 134 b}$, H. Akerstedt ${ }^{147 a, 147 b}$, T.P.A. Åkesson ${ }^{80}$, G. Akimoto ${ }^{156}$, A.V. Akimov ${ }^{95}$, G.L. Alberghi $20 a, 20 b$, J. Albert ${ }^{170}$, S. Albrand ${ }^{55}$, M.J. Alconada Verzini ${ }^{70}$, M. Aleksa ${ }^{30}$, I.N. Aleksandrov ${ }^{64}$, C. Alexa ${ }^{26 a}$, G. Alexander ${ }^{154}$, G. Alexandre ${ }^{49}$, T. Alexopoulos ${ }^{10}$, M. Alhroob ${ }^{165 a, 165 c}$, G. Alimonti ${ }^{90 a}$, L. Alio ${ }^{84}$, J. Alison ${ }^{31}$, B.M.M. Allbrooke ${ }^{18}$, L.J. Allison ${ }^{71}$, P.P. Allport ${ }^{73}$, J. Almond ${ }^{83}$, A. Aloisio 103a,103b, A. Alonso ${ }^{36}$, F. Alonso ${ }^{70}$, C. Alpigiani ${ }^{75}$, A. Altheimer ${ }^{35}$, B. Alvarez Gonzalez ${ }^{89}$, M.G. Alviggi 103a,103b,

K. Amako ${ }^{65}$, Y. Amaral Coutinho ${ }^{24 a}$, C. Amelung ${ }^{23}$, D. Amidei ${ }^{88}$, S.P. Amor Dos Santos ${ }^{125 a, 125 c}$, A. Amorim $125 \mathrm{a}, 125 \mathrm{~b}, \mathrm{~S}$. Amoroso ${ }^{48}$, N. Amram ${ }^{154}$, G. Amundsen ${ }^{23}$, C. Anastopoulos ${ }^{140}$, L.S. Ancu $^{49}$, N. Andari ${ }^{30}$, T. Andeen ${ }^{35}$, C.F. Anders ${ }^{58 b}$, G. Anders ${ }^{30}$, K.J. Anderson ${ }^{31}$, A. Andreazza ${ }^{90 a, 90 b}$, V. Andrei ${ }^{58 a}$, X.S. Anduaga ${ }^{70}$, S. Angelidakis ${ }^{9}$, I. Angelozzi ${ }^{106}$, P. Anger $^{44}$, A. Angerami ${ }^{35}$, F. Anghinolfi ${ }^{30}$, A.V. Anisenkov ${ }^{108}$, N. Anjos ${ }^{125 a}$, A. Annovi ${ }^{47}$, A. Antonaki ${ }^{9}$, M. Antonelli ${ }^{47}$, A. Antonov ${ }^{97}$, J. Antos ${ }^{145 b}$, F. Anulli ${ }^{133 a}$, M. Aoki ${ }^{65}$, L. Aperio Bella ${ }^{18}$, R. Apolle ${ }^{119, c}$, G. Arabidze ${ }^{89}$, I. Aracena ${ }^{144}$, Y. Arai ${ }^{65}$, J.P. Araque ${ }^{125 a}$, A.T.H. Arce ${ }^{45}$, J-F. Arguin ${ }^{94}$, S. Argyropoulos ${ }^{42}$, M. Arik ${ }^{19 a}$, A.J. Armbruster ${ }^{30}$, O. Arnaez ${ }^{30}$, V. Arnal ${ }^{81}$, H. Arnold ${ }^{48}$, M. Arratia ${ }^{28}$, O. Arslan ${ }^{21}$, A. Artamonov ${ }^{96}$, G. Artoni ${ }^{23}$, S. Asai ${ }^{156}$, N. Asbah ${ }^{42}$, A. Ashkenazi ${ }^{154}$, B. Åsman 147a,147b, L. Asquith 6 , K. Assamagan ${ }^{25}$, R. Astalos ${ }^{145 a}$,

M. Atkinson ${ }^{166}$, N.B. Atlay ${ }^{142}$, B. Auerbach ${ }^{6}$, K. Augsten ${ }^{127}$, M. Aurousseau ${ }^{146 b}$, G. Avolio ${ }^{30}$, G. Azuelos ${ }^{94, d}$, Y. Azuma ${ }^{156}$, M.A. Baak ${ }^{30}$, A. Baas ${ }^{58 a}$, C. Bacci ${ }^{135 a, 135 b}$, H. Bachacou ${ }^{137}$, K. Bachas ${ }^{155}$, M. Backes ${ }^{30}$, M. Backhaus ${ }^{30}$, J. Backus Mayes ${ }^{144}$, E. Badescu ${ }^{26 a}$, P. Bagiacchi133a,133b, P. Bagnaia ${ }^{133 a, 133 b}$, Y. Bai ${ }^{33 a}$, T. Bain ${ }^{35}$, J.T. Baines ${ }^{130}$, O.K. Baker ${ }^{177}$, P. Balek ${ }^{128}$, F. Balli ${ }^{137}$, E. Banas ${ }^{39}$, Sw. Banerjee ${ }^{174}$, A.A.E. Bannoura ${ }^{176}$, V. Bansal ${ }^{170}$, H.S. Bansil ${ }^{18}$, L. Barak ${ }^{173}$, S.P. Baranov ${ }^{95}$, E.L. Barberio ${ }^{87}$, D. Barberis ${ }^{50 a, 50 b}$, M. Barbero ${ }^{84}$, T. Barillari ${ }^{100}$, M. Barisonzi ${ }^{176}$, T. Barklow ${ }^{144}$, N. Barlow ${ }^{28}$, B.M. Barnett ${ }^{130}$, R.M. Barnett ${ }^{15}$, Z. Barnovska ${ }^{5}$, A. Baroncelli ${ }^{135 a}$, G. Barone ${ }^{49}$, A.J. Barr ${ }^{119}$, F. Barreiro ${ }^{81}$,

J. Barreiro Guimarães da Costa ${ }^{57}$, R. Bartoldus ${ }^{144}$, A.E. Barton ${ }^{71}$, P. Bartos ${ }^{145 a}$, V. Bartsch ${ }^{150}$, A. Bassalat ${ }^{116}$, A. Basye ${ }^{166}$, R.L. Bates ${ }^{53}$, L. Batkova ${ }^{145 a}$, J.R. Batley ${ }^{28}$, M. Battaglia ${ }^{138}$, M. Battistin ${ }^{30}$, F. Bauer ${ }^{137}$, H.S. Bawa ${ }^{144, e}$, T. Beau ${ }^{79}$, P.H. Beauchemin ${ }^{162}$,

R. Beccherle ${ }^{123 a, 123 b}$, P. Bechtle ${ }^{21}$, H.P. Beck ${ }^{17}$, K. Becker ${ }^{176}$, S. Becker ${ }^{99}$, M. Beckingham ${ }^{171}$, C. Becot $^{116}$, A.J. Beddall ${ }^{19 c}$, A. Beddall ${ }^{19 c}$, S. Bedikian ${ }^{177}$, V.A. Bednyakov ${ }^{64}$, C.P. Bee ${ }^{149}$, L.J. Beemster ${ }^{106}$, T.A. Beermann ${ }^{176}$, M. Begel ${ }^{25}$, K. Behr ${ }^{119}$, C. Belanger-Champagne ${ }^{86}$, P.J. Bell ${ }^{49}$, W.H. Bell ${ }^{49}$, G. Bella ${ }^{154}$, L. Bellagamba ${ }^{20 a}$, A. Bellerive $^{29}$, M. Bellomo ${ }^{85}$, K. Belotskiy ${ }^{97}$, O. Beltramello ${ }^{30}$, O. Benary ${ }^{154}$, D. Benchekroun ${ }^{136 a}$, K. Bendtz ${ }^{147 a, 147 b}$, N. Benekos ${ }^{166}$, Y. Benhammou ${ }^{154}$, E. Benhar Noccioli ${ }^{49}$, J.A. Benitez Garcia ${ }^{160 b}$, D.P. Benjamin ${ }^{45}$, J.R. Bensinger ${ }^{23}$, K. Benslama ${ }^{131}$, S. Bentvelsen ${ }^{106}$, D. Berge ${ }^{106}$, E. Bergeaas Kuutmann ${ }^{16}$, N. Berger ${ }^{5}$, F. Berghaus ${ }^{170}$, J. Beringer ${ }^{15}$, C. Bernard ${ }^{22}$, P. Bernat ${ }^{77}$, C. Bernius ${ }^{78}$, F.U. Bernlochner ${ }^{170}$, T. Berry ${ }^{76}$, P. Berta ${ }^{128}$, C. Bertella ${ }^{84}$, G. Bertoli ${ }^{147 a, 147 b}$, F. Bertolucci ${ }^{123 a, 123 b}$, D. Bertsche ${ }^{112}$, M.I. Besana ${ }^{90 a}$, G.J. Besjes ${ }^{105}$, O. Bessidskaia ${ }^{147 a, 147 b}$, M.F. Bessner ${ }^{42}$, N. Besson ${ }^{137}$, C. Betancourt ${ }^{48}$, S. Bethke ${ }^{100}$, W. Bhimji ${ }^{46}$, R.M. Bianchi ${ }^{124}$, L. Bianchini ${ }^{23}$, M. Bianco ${ }^{30}$, O. Biebel ${ }^{99}$, S.P. Bieniek ${ }^{77}$, K. Bierwagen ${ }^{54}$, J. Biesiada ${ }^{15}$, M. Biglietti ${ }^{135 a}$, J. Bilbao De Mendizabal ${ }^{49}$, H. Bilokon ${ }^{47}$, M. Bindi ${ }^{54}$, S. Binet ${ }^{116}$, A. Bingul ${ }^{19 c}$, C. Bini 133a,133b, C.W. Black ${ }^{151}$, J.E. Black ${ }^{144}$, K.M. Black ${ }^{22}$, D. Blackburn ${ }^{139}$, R.E. Blair ${ }^{6}$, J.-B. Blanchard ${ }^{137}$, T. Blazek ${ }^{145 a}$, I. Bloch ${ }^{42}$, C. Blocker ${ }^{23}$, W. Blum ${ }^{82, *}$, U. Blumenschein ${ }^{54}$, 
G.J. Bobbink ${ }^{106}$, V.S. Bobrovnikov ${ }^{108}$, S.S. Bocchetta ${ }^{80}$, A. Bocci ${ }^{45}$, C. Bock ${ }^{99}$, C.R. Boddy ${ }^{119}$, M. Boehler ${ }^{48}$, T.T. Boek ${ }^{176}$, J.A. Bogaerts ${ }^{30}$, A.G. Bogdanchikov ${ }^{108}$, A. Bogouch ${ }^{91, *}$,

C. Bohm ${ }^{147 a}$, J. Bohm ${ }^{126}$, V. Boisvert ${ }^{76}$, T. Bold ${ }^{38 a}$, V. Boldea ${ }^{26 a}$, A.S. Boldyrev ${ }^{98}$,

M. Bomben ${ }^{79}$, M. Bona ${ }^{75}$, M. Boonekamp ${ }^{137}$, A. Borisov ${ }^{129}$, G. Borissov ${ }^{71}$, M. Borri ${ }^{83}$,

S. Borroni ${ }^{42}$, J. Bortfeldt ${ }^{99}$, V. Bortolotto ${ }^{135 a, 135 b}$, K. Bos ${ }^{106}$, D. Boscherini ${ }^{20 a}$, M. Bosman ${ }^{12}$,

H. Boterenbrood ${ }^{106}$, J. Boudreau ${ }^{124}$, J. Bouffard ${ }^{2}$, E.V. Bouhova-Thacker ${ }^{71}$, D. Boumediene ${ }^{34}$,

C. Bourdarios ${ }^{116}$, N. Bousson ${ }^{113}$, S. Boutouil ${ }^{136 d}$, A. Boveia ${ }^{31}$, J. Boyd ${ }^{30}$, I.R. Boyko ${ }^{64}$,

J. Bracinik ${ }^{18}$, A. Brandt ${ }^{8}$, G. Brandt ${ }^{15}$, O. Brandt ${ }^{58 a}$, U. Bratzler ${ }^{157}$, B. Brau ${ }^{85}$, J.E. Brau ${ }^{115}$, H.M. Braun ${ }^{176, *}$, S.F. Brazzale ${ }^{165 a, 165 c}$, B. Brelier ${ }^{159}$, K. Brendlinger ${ }^{121}$, A.J. Brennan ${ }^{87}$,

R. Brenner ${ }^{167}$, S. Bressler ${ }^{173}$, K. Bristow ${ }^{146 c}$, T.M. Bristow ${ }^{46}$, D. Britton ${ }^{53}$, F.M. Brochu ${ }^{28}$,

I. Brock ${ }^{21}$, R. Brock ${ }^{89}$, C. Bromberg ${ }^{89}$, J. Bronner ${ }^{100}$, G. Brooijmans ${ }^{35}$, T. Brooks ${ }^{76}$,

W.K. Brooks ${ }^{32 b}$, J. Brosamer ${ }^{15}$, E. Brost ${ }^{115}$, J. Brown ${ }^{55}$, P.A. Bruckman de Renstrom ${ }^{39}$,

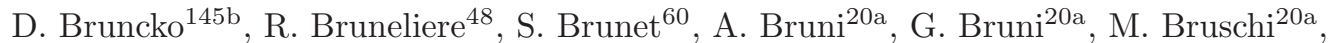

L. Bryngemark ${ }^{80}$, T. Buanes ${ }^{14}$, Q. Buat ${ }^{143}$, F. Bucci ${ }^{49}$, P. Buchholz ${ }^{142}$, R.M. Buckingham ${ }^{119}$,

A.G. Buckley ${ }^{53}$, S.I. Buda ${ }^{26 a}$, I.A. Budagov ${ }^{64}$, F. Buehrer ${ }^{48}$, L. Bugge ${ }^{118}$, M.K. Bugge ${ }^{118}$,

O. Bulekov ${ }^{97}$, A.C. Bundock ${ }^{73}$, H. Burckhart ${ }^{30}$, S. Burdin ${ }^{73}$, B. Burghgrave ${ }^{107}$, S. Burke ${ }^{130}$,

I. Burmeister ${ }^{43}$, E. Busato ${ }^{34}$, D. Büscher ${ }^{48}$, V. Büscher ${ }^{82}$, P. Bussey ${ }^{53}$, C.P. Buszello ${ }^{167}$,

B. Butler ${ }^{57}$, J.M. Butler ${ }^{22}$, A.I. Butt ${ }^{3}$, C.M. Buttar ${ }^{53}$, J.M. Butterworth ${ }^{77}$, P. Butti ${ }^{106}$,

W. Buttinger ${ }^{28}$, A. Buzatu ${ }^{53}$, M. Byszewski ${ }^{10}$, S. Cabrera Urbán ${ }^{168}$, D. Caforio ${ }^{20 a, 20 b}$, O. Cakir ${ }^{4 a}$,

P. Calafiura ${ }^{15}$, A. Calandri ${ }^{137}$, G. Calderini ${ }^{79}$, P. Calfayan ${ }^{99}$, R. Calkins ${ }^{107}$, L.P. Caloba ${ }^{24 a}$,

D. Calvet ${ }^{34}$, S. Calvet ${ }^{34}$, R. Camacho Toro ${ }^{49}$, S. Camarda ${ }^{42}$, D. Cameron ${ }^{118}$, L.M. Caminada ${ }^{15}$,

R. Caminal Armadans ${ }^{12}$, S. Campana ${ }^{30}$, M. Campanelli ${ }^{77}$, A. Campoverde ${ }^{149}$, V. Canale ${ }^{103 a, 103 b}$,

A. Canepa ${ }^{160 a}$, M. Cano Bret ${ }^{75}$, J. Cantero ${ }^{81}$, R. Cantrill ${ }^{76}$, T. Cao ${ }^{40}$,

M.D.M. Capeans Garrido ${ }^{30}$, I. Caprini ${ }^{26 a}$, M. Caprini ${ }^{26 a}$, M. Capua ${ }^{37 a, 37 b}$, R. Caputo ${ }^{82}$,

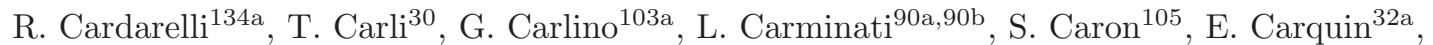

G.D. Carrillo-Montoya ${ }^{146 c}$, J.R. Carter ${ }^{28}$, J. Carvalho ${ }^{125 a, 125 c}$, D. Casadei ${ }^{77}$, M.P. Casado ${ }^{12}$,

M. Casolino ${ }^{12}$, E. Castaneda-Miranda ${ }^{146 b}$, A. Castelli ${ }^{106}$, V. Castillo Gimenez ${ }^{168}$, N.F. Castro ${ }^{125 a}$,

P. Catastini ${ }^{57}$, A. Catinaccio ${ }^{30}$, J.R. Catmore ${ }^{118}$, A. Cattai ${ }^{30}$, G. Cattani ${ }^{134 a, 134 b}$, S. Caughron ${ }^{89}$,

V. Cavaliere ${ }^{166}$, D. Cavalli ${ }^{90 a}$, M. Cavalli-Sforza ${ }^{12}$, V. Cavasinni ${ }^{123 a, 123 b}$, F. Ceradini ${ }^{135 a, 135 b}$,

B. Cerio ${ }^{45}$, K. Cerny ${ }^{128}$, A.S. Cerqueira ${ }^{24 b}$, A. Cerri ${ }^{150}$, L. Cerrito ${ }^{75}$, F. Cerutti ${ }^{15}$, M. Cerv ${ }^{30}$,

A. Cervelli ${ }^{17}$, S.A. Cetin ${ }^{19 b}$, A. Chafaq ${ }^{136 a}$, D. Chakraborty ${ }^{107}$, I. Chalupkova ${ }^{128}$, P. Chang ${ }^{166}$,

B. Chapleau ${ }^{86}$, J.D. Chapman ${ }^{28}$, D. Charfeddine ${ }^{116}$, D.G. Charlton ${ }^{18}$, C.C. Chau ${ }^{159}$,

C.A. Chavez Barajas ${ }^{150}$, S. Cheatham ${ }^{86}$, A. Chegwidden ${ }^{89}$, S. Chekanov ${ }^{6}$, S.V. Chekulaev ${ }^{160 a}$,

G.A. Chelkov ${ }^{64, f}$, M.A. Chelstowska ${ }^{88}$, C. Chen ${ }^{63}$, H. Chen ${ }^{25}$, K. Chen ${ }^{149}$, L. Chen ${ }^{33 d, g}$,

S. Chen ${ }^{33 c}$, X. Chen ${ }^{146 c}$, Y. Chen ${ }^{35}$, H.C. Cheng ${ }^{88}$, Y. Cheng ${ }^{31}$, A. Cheplakov ${ }^{64}$,

R. Cherkaoui El Moursli ${ }^{136 e}$, V. Chernyatin ${ }^{25, *}$ E. Cheu ${ }^{7}$, L. Chevalier ${ }^{137}$, V. Chiarella ${ }^{47}$,

G. Chiefari ${ }^{103 a}, 103 b$, J.T. Childers ${ }^{6}$, A. Chilingarov ${ }^{71}$, G. Chiodini ${ }^{72 a}$, A.S. Chisholm ${ }^{18}$,

R.T. Chislett ${ }^{77}$, A. Chitan ${ }^{26 a}$, M.V. Chizhov ${ }^{64}$, S. Chouridou ${ }^{9}$, B.K.B. Chow ${ }^{99}$,

D. Chromek-Burckhart ${ }^{30}$, M.L. Chu ${ }^{152}$, J. Chudoba ${ }^{126}$, J.J. Chwastowski ${ }^{39}$, L. Chytka ${ }^{114}$,

G. Ciapetti ${ }^{133 a}$,133b, A.K. Ciftci ${ }^{4 a}$, R. Ciftci ${ }^{4 a}$, D. Cinca ${ }^{53}$, V. Cindro ${ }^{74}$, A. Ciocio ${ }^{15}$,

P. Cirkovic ${ }^{13 b}$, Z.H. Citron ${ }^{173}$, M. Citterio ${ }^{90 a}$, M. Ciubancan ${ }^{26 a}$, A. Clark ${ }^{49}$, P.J. Clark ${ }^{46}$,

R.N. Clarke ${ }^{15}$, W. Cleland ${ }^{124}$, J.C. Clemens ${ }^{84}$, C. Clement ${ }^{147 a, 147 b}$, Y. Coadou ${ }^{84}$,

M. Cobal ${ }^{165 a, 165 c}$, A. Coccaro ${ }^{139}$, J. Cochran $^{63}$, L. Coffey ${ }^{23}$, J.G. Cogan ${ }^{144}$, J. Coggeshall ${ }^{166}$,

B. Cole ${ }^{35}$, S. Cole ${ }^{107}$, A.P. Colijn ${ }^{106}$, J. Collot ${ }^{55}$, T. Colombo ${ }^{58 c}$, G. Colon ${ }^{85}$, G. Compostella ${ }^{100}$,

P. Conde Muiño ${ }^{125 a, 125 b}$, E. Coniavitis ${ }^{48}$, M.C. Conidi ${ }^{12}$, S.H. Connell ${ }^{146 b}$, I.A. Connelly ${ }^{76}$,

S.M. Consonni ${ }^{90 a}, 90 b$, V. Consorti ${ }^{48}$, S. Constantinescu ${ }^{26 a}$, C. Conta ${ }^{120 a, 120 b}$, G. Conti ${ }^{57}$,

F. Conventi ${ }^{103 a}, h$, M. Cooke ${ }^{15}$, B.D. Cooper ${ }^{77}$, A.M. Cooper-Sarkar ${ }^{119}$, N.J. Cooper-Smith ${ }^{76}$,

K. Copic ${ }^{15}$, T. Cornelissen ${ }^{176}$, M. Corradi ${ }^{20 a}$, F. Corriveau ${ }^{86, i}$, A. Corso-Radu ${ }^{164}$, 
A. Cortes-Gonzalez ${ }^{12}$, G. Cortiana ${ }^{100}$, G. Costa ${ }^{90 a}$, M.J. Costa ${ }^{168}$, D. Costanzo ${ }^{140}$, D. Côté ${ }^{8}$, G. Cottin $^{28}$, G. Cowan ${ }^{76}$, B.E. $\mathrm{Cox}^{83}$, K. Cranmer ${ }^{109}$, G. Cree ${ }^{29}$, S. Crépé-Renaudin ${ }^{55}$,

F. Crescioli ${ }^{79}$, W.A. Cribbs ${ }^{147 a, 147 b}$, M. Crispin Ortuzar ${ }^{119}$, M. Cristinziani ${ }^{21}$, V. Croft $^{105}$,

G. Crosetti ${ }^{37 a, 37 b}$, C.-M. Cuciuc ${ }^{26 a}$, T. Cuhadar Donszelmann ${ }^{140}$, J. Cummings ${ }^{177}$,

M. Curatolo ${ }^{47}$, C. Cuthbert ${ }^{151}$, H. Czirr ${ }^{142}$, P. Czodrowski ${ }^{3}$, Z. Czyczula ${ }^{177}$, S. D'Auria ${ }^{53}$,

M. D'Onofrio ${ }^{73}$, M.J. Da Cunha Sargedas De Sousa ${ }^{125 a, 125 b}$, C. Da Via ${ }^{83}$, W. Dabrowski ${ }^{38 a}$,

A. Dafinca ${ }^{119}$, T. Dai ${ }^{88}$, O. Dale ${ }^{14}$, F. Dallaire ${ }^{94}$, C. Dallapiccola ${ }^{85}$, M. Dam ${ }^{36}$, A.C. Daniells ${ }^{18}$,

M. Dano Hoffmann ${ }^{137}$, V. Dao ${ }^{105}$, G. Darbo ${ }^{50 a}$, S. Darmora ${ }^{8}$, J.A. Dassoulas ${ }^{42}$, A. Dattagupta ${ }^{60}$,

W. Davey ${ }^{21}$, C. David ${ }^{170}$, T. Davidek ${ }^{128}$, E. Davies ${ }^{119, c}$, M. Davies ${ }^{154}$, O. Davignon ${ }^{79}$,

A.R. Davison ${ }^{77}$, P. Davison ${ }^{77}$, Y. Davygora ${ }^{58 a}$, E. Dawe ${ }^{143}$, I. Dawson ${ }^{140}$,

R.K. Daya-Ishmukhametova ${ }^{85}$, K. De ${ }^{8}$, R. de Asmundis ${ }^{103 a}$, S. De Castro ${ }^{20 a, 20 b}$, S. De Cecco ${ }^{79}$,

N. De Groot ${ }^{105}$, P. de Jong ${ }^{106}$, H. De la Torre ${ }^{81}$, F. De Lorenzi ${ }^{63}$, L. De Nooij ${ }^{106}$, D. De Pedis ${ }^{133 a}$,

A. De Salvo ${ }^{133 a}$, U. De Sanctis ${ }^{165 a, 165 b}$, A. De Santo ${ }^{150}$, J.B. De Vivie De Regie ${ }^{116}$,

W.J. Dearnaley ${ }^{71}$, R. Debbe ${ }^{25}$, C. Debenedetti ${ }^{138}$, B. Dechenaux ${ }^{55}$, D.V. Dedovich ${ }^{64}$,

I. Deigaard ${ }^{106}$, J. Del Peso ${ }^{81}$, T. Del Prete ${ }^{123 a, 123 b}$, F. Deliot ${ }^{137}$, C.M. Delitzsch ${ }^{49}$,

M. Deliyergiyev ${ }^{74}$, A. Dell'Acqua ${ }^{30}$, L. Dell'Asta ${ }^{22}$, M. Dell'Orso ${ }^{123 a, 123 b}$, M. Della Pietra ${ }^{103 a, h}$,

D. della Volpe ${ }^{49}$, M. Delmastro ${ }^{5}$, P.A. Delsart ${ }^{55}$, C. Deluca ${ }^{106}$, S. Demers ${ }^{177}$, M. Demichev ${ }^{64}$,

A. Demilly ${ }^{79}$, S.P. Denisov ${ }^{129}$, D. Derendarz ${ }^{39}$, J.E. Derkaoui ${ }^{136 d}$, F. Derue ${ }^{79}$, P. Dervan ${ }^{73}$,

K. Desch $^{21}$, C. Deterre ${ }^{42}$, P.O. Deviveiros ${ }^{106}$, A. Dewhurst ${ }^{130}$, S. Dhaliwal ${ }^{106}$,

A. Di Ciaccio ${ }^{134 a, 134 b}$, L. Di Ciaccio ${ }^{5}$, A. Di Domenico ${ }^{133 a, 133 b}$, C. Di Donato ${ }^{103 a, 103 b}$,

A. Di Girolamo ${ }^{30}$, B. Di Girolamo ${ }^{30}$, A. Di Mattia ${ }^{153}$, B. Di Micco ${ }^{135 a, 135 b}$, R. Di Nardo ${ }^{47}$,

A. Di Simone ${ }^{48}$, R. Di $\operatorname{Sipio}^{20 a}$ 20b, D. Di Valentino ${ }^{29}$, F.A. Dias ${ }^{46}$, M.A. Diaz ${ }^{32 a}$, E.B. Diehl ${ }^{88}$,

J. Dietrich ${ }^{42}$, T.A. Dietzsch ${ }^{58 a}$, S. Diglio ${ }^{84}$, A. Dimitrievska ${ }^{13 a}$, J. Dingfelder ${ }^{21}$,

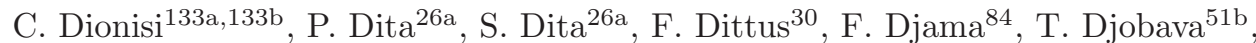

M.A.B. do Vale ${ }^{24 c}$, A. Do Valle Wemans ${ }^{125 a, 125 g}$, T.K.O. Doan ${ }^{5}$, D. Dobos ${ }^{30}$, C. Doglioni ${ }^{49}$,

T. Doherty ${ }^{53}$, T. Dohmae ${ }^{156}$, J. Dolejsi ${ }^{128}$, Z. Dolezal ${ }^{128}$, B.A. Dolgoshein ${ }^{97, *}$, M. Donadelli2 ${ }^{24 d}$,

S. Donati ${ }^{123 a, 123 b}$, P. Dondero ${ }^{120 a, 120 b}$, J. Donini ${ }^{34}$, J. Dopke ${ }^{130}$, A. Doria ${ }^{103 a}$, M.T. Dova ${ }^{70}$,

A.T. Doyle ${ }^{53}$, M. Dris ${ }^{10}$, J. Dubbert ${ }^{88}$, S. Dube ${ }^{15}$, E. Dubreuil ${ }^{34}$, E. Duchovni ${ }^{173}$, G. Duckeck ${ }^{99}$,

O.A. Ducu ${ }^{26 a}$, D. Duda ${ }^{176}$, A. Dudarev ${ }^{30}$, F. Dudziak ${ }^{63}$, L. Duflot ${ }^{116}$, L. Duguid ${ }^{76}$,

M. Dührssen ${ }^{30}$, M. Dunford ${ }^{58 a}$, H. Duran Yildiz ${ }^{4 a}$, M. Düren ${ }^{52}$, A. Durglishvili ${ }^{51 b}$,

M. Dwuznik ${ }^{38 a}$, M. Dyndal ${ }^{38 a}$, J. Ebke ${ }^{99}$, W. Edson ${ }^{2}$, N.C. Edwards ${ }^{46}$, W. Ehrenfeld ${ }^{21}$,

T. Eifert ${ }^{144}$, G. Eigen ${ }^{14}$, K. Einsweiler ${ }^{15}$, T. Ekelof ${ }^{167}$, M. El Kacimi ${ }^{136 c}$, M. Ellert ${ }^{167}$, S. Elles ${ }^{5}$,

F. Ellinghaus ${ }^{82}$, N. Ellis ${ }^{30}$, J. Elmsheuser ${ }^{99}$, M. Elsing ${ }^{30}$, D. Emeliyanov ${ }^{130}$, Y. Enari ${ }^{156}$,

O.C. Endner ${ }^{82}$, M. Endo ${ }^{117}$, R. Engelmann ${ }^{149}$, J. Erdmann ${ }^{177}$, A. Ereditato ${ }^{17}$, D. Eriksson ${ }^{147 a}$,

G. Ernis ${ }^{176}$, J. Ernst ${ }^{2}$, M. Ernst ${ }^{25}$, J. Ernwein ${ }^{137}$, D. Errede ${ }^{166}$, S. Errede ${ }^{166}$, E. Ertel ${ }^{82}$,

M. Escalier ${ }^{116}$, H. $\operatorname{Esch}^{43}$, C. Escobar ${ }^{124}$, B. Esposito ${ }^{47}$, A.I. Etienvre ${ }^{137}$, E. Etzion ${ }^{154}$,

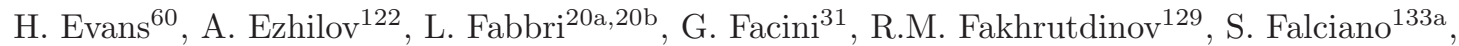

R.J. Falla ${ }^{77}$, J. Faltova ${ }^{128}$, Y. Fang ${ }^{33 a}$, M. Fanti ${ }^{90 a, 90 b}$, A. Farbin ${ }^{8}$, A. Farilla ${ }^{135 a}$, T. Farooque ${ }^{12}$,

S. Farrell ${ }^{164}$, S.M. Farrington ${ }^{171}$, P. Farthouat ${ }^{30}$, F. Fassi ${ }^{168}$, P. Fassnacht ${ }^{30}$, D. Fassouliotis ${ }^{9}$,

A. Favareto ${ }^{50 a, 50 b}$, L. Fayard ${ }^{116}$, P. Federic ${ }^{145 a}$, O.L. Fedin ${ }^{122, j}$, W. Fedorko ${ }^{169}$,

M. Fehling-Kaschek ${ }^{48}$, S. Feigl ${ }^{30}$, L. Feligioni ${ }^{84}$, C. Feng ${ }^{33 d}$, E.J. Feng ${ }^{6}$, H. Feng ${ }^{88}$,

A.B. Fenyuk ${ }^{129}$, S. Fernandez Perez $^{30}$, S. Ferrag ${ }^{53}$, J. Ferrando ${ }^{53}$, A. Ferrari ${ }^{167}$, P. Ferrari ${ }^{106}$,

R. Ferrari ${ }^{120 a}$, D.E. Ferreira de Lima ${ }^{53}$, A. Ferrer ${ }^{168}$, D. Ferrere ${ }^{49}$, C. Ferretti ${ }^{88}$,

A. Ferretto Parodi ${ }^{50 a, 50 b}$, M. Fiascaris ${ }^{31}$, F. Fiedler ${ }^{82}$, A. Filipčic ${ }^{74}$, M. Filipuzzi ${ }^{42}$, F. Filthaut ${ }^{105}$,

M. Fincke-Keeler ${ }^{170}$, K.D. Finelli ${ }^{151}$, M.C.N. Fiolhais ${ }^{125 a, 125 c}$, L. Fiorini ${ }^{168}$, A. Firan ${ }^{40}$,

A. Fischer ${ }^{2}$, J. Fischer ${ }^{176}$, W.C. Fisher ${ }^{89}$, E.A. Fitzgerald ${ }^{23}$, M. Flechl ${ }^{48}$, I. Fleck ${ }^{142}$,

P. Fleischmann ${ }^{88}$, S. Fleischmann ${ }^{176}$, G.T. Fletcher ${ }^{140}$, G. Fletcher ${ }^{75}$, T. Flick ${ }^{176}$, A. Floderus ${ }^{80}$, L.R. Flores Castillo ${ }^{174, k}$, A.C. Florez Bustos ${ }^{160 b}$, M.J. Flowerdew ${ }^{100}$, A. Formica ${ }^{137}$, A. Forti ${ }^{83}$, 
D. Fortin ${ }^{160 a}$, D. Fournier ${ }^{116}$, H. Fox ${ }^{71}$, S. Fracchia ${ }^{12}$, P. Francavilla ${ }^{79}$, M. Franchini20a,20b, S. Franchino ${ }^{30}$, D. Francis ${ }^{30}$, M. Franklin ${ }^{57}$, S. Franz ${ }^{61}$, M. Fraternali ${ }^{120 a, 120 b}$, S.T. French ${ }^{28}$, C. Friedrich ${ }^{42}$, F. Friedrich ${ }^{44}$, D. Froidevaux ${ }^{30}$, J.A. Frost ${ }^{28}$, C. Fukunaga ${ }^{157}$,

E. Fullana Torregrosa ${ }^{82}$, B.G. Fulsom ${ }^{144}$, J. Fuster ${ }^{168}$, C. Gabaldon ${ }^{55}$, O. Gabizon ${ }^{173}$, A. Gabrielli ${ }^{20 a, 20 b}$, A. Gabrielli ${ }^{133 a, 133 b}$, S. Gadatsch ${ }^{106}$, S. Gadomski ${ }^{49}$, G. Gagliardi ${ }^{50 a, 50 b}$, P. Gagnon ${ }^{60}$, C. Galea ${ }^{105}$, B. Galhardo ${ }^{125 a, 125 c}$, E.J. Gallas ${ }^{119}$, V. Gallo ${ }^{17}$, B.J. Gallop ${ }^{130}$, P. Gallus ${ }^{127}$, G. Galster ${ }^{36}$, K.K. Gan ${ }^{110}$, R.P. Gandrajula ${ }^{62}$, J. Gao ${ }^{33 b, g}$, Y.S. Gao ${ }^{144, e}$, F.M. Garay Walls ${ }^{46}$, F. Garberson ${ }^{177}$, C. García ${ }^{168}$, J.E. García Navarro ${ }^{168}$, M. Garcia-Sciveres ${ }^{15}$, R.W. Gardner ${ }^{31}$, N. Garelli ${ }^{144}$, V. Garonne ${ }^{30}$, C. Gatti ${ }^{47}$, G. Gaudio ${ }^{120 a}$, B. Gaur ${ }^{142}$, L. Gauthier ${ }^{94}$, P. Gauzzi ${ }^{133 a, 133 b}$, I.L. Gavrilenko ${ }^{95}$, C. Gay ${ }^{169}$, G. Gaycken ${ }^{21}$, E.N. Gazis ${ }^{10}$, P. Ge ${ }^{33 d}$, Z. Gecse ${ }^{169}$, C.N.P. Gee ${ }^{130}$, D.A.A. Geerts ${ }^{106}$, Ch. Geich-Gimbel ${ }^{21}$,

K. Gellerstedt ${ }^{147 a, 147 b}$, C. Gemme ${ }^{50 a}$, A. Gemmell ${ }^{53}$, M.H. Genest ${ }^{55}$, S. Gentile ${ }^{133 a, 133 b}$, M. George ${ }^{54}$, S. George ${ }^{76}$, D. Gerbaudo ${ }^{164}$, A. Gershon ${ }^{154}$, H. Ghazlane ${ }^{136 b}$, N. Ghodbane ${ }^{34}$, B. Giacobbe ${ }^{20 a}$, S. Giagu ${ }^{133 a, 133 b}$, V. Giangiobbe ${ }^{12}$, P. Giannetti ${ }^{123 a, 123 b}$, F. Gianotti ${ }^{30}$, B. Gibbard ${ }^{25}$, S.M. Gibson ${ }^{76}$, M. Gilchriese ${ }^{15}$, T.P.S. Gillam ${ }^{28}$, D. Gillberg ${ }^{30}$, G. Gilles ${ }^{34}$, D.M. Gingrich ${ }^{3, d}$, N. Giokaris ${ }^{9}$, M.P. Giordani ${ }^{165 a, 165 c}$, R. Giordano ${ }^{103 a, 103 b}$, F.M. Giorgi ${ }^{20 a}$, F.M. Giorgi ${ }^{16}$, P.F. Giraud ${ }^{137}$, D. Giugni ${ }^{90 a}$, C. Giuliani ${ }^{48}$, M. Giulini ${ }^{58 b}$, B.K. Gjelsten ${ }^{118}$, S. Gkaitatzis ${ }^{155}$, I. Gkialas ${ }^{155, l}$, L.K. Gladilin ${ }^{98}$, C. Glasman ${ }^{81}$, J. Glatzer ${ }^{30}$, P.C.F. Glaysher ${ }^{46}$, A. Glazov ${ }^{42}$, G.L. Glonti ${ }^{64}$, M. Goblirsch-Kolb ${ }^{100}$, J.R. Goddard ${ }^{75}$, J. Godfrey ${ }^{143}$, J. Godlewski ${ }^{30}$, C. Goeringer ${ }^{82}$, S. Goldfarb ${ }^{88}$, T. Golling ${ }^{177}$, D. Golubkov ${ }^{129}$, A. Gomes ${ }^{125 a, 125 b, 125 d}$, L.S. Gomez Fajardo ${ }^{42}$, R. Gonçalo ${ }^{125 a}$, J. Goncalves Pinto Firmino Da Costa ${ }^{137}$, L. Gonella ${ }^{21}$, S. González de la Hoz ${ }^{168}$, G. Gonzalez Parra ${ }^{12}$, S. Gonzalez-Sevilla ${ }^{49}$, L. Goossens ${ }^{30}$, P.A. Gorbounov ${ }^{96}$, H.A. Gordon ${ }^{25}$, I. Gorelov ${ }^{104}$, B. Gorini ${ }^{30}$, E. Gorini ${ }^{72 a, 72 b}$, A. Gorišek ${ }^{74}$, E. Gornicki ${ }^{39}$, A.T. Goshaw ${ }^{6}$, C. Gössling ${ }^{43}$, M.I. Gostkin ${ }^{64}$, M. Gouighri136a ${ }^{13}$ D. Goujdami ${ }^{136 c}$, M.P. Goulette ${ }^{49}$, A.G. Goussiou ${ }^{139}$, C. Goy ${ }^{5}$, S. Gozpinar ${ }^{23}$, H.M.X. Grabas ${ }^{137}$, L. Graber ${ }^{54}$, I. Grabowska-Bold ${ }^{38 a}$, P. Grafström ${ }^{20 a, 20 b}$, K-J. Grahn ${ }^{42}$, J. Gramling ${ }^{49}$, E. Gramstad ${ }^{118}$, S. Grancagnolo ${ }^{16}$, V. Grassi ${ }^{149}$, V. Gratchev ${ }^{122}$, H.M. Gray ${ }^{30}$, E. Graziani ${ }^{135 a}$, O.G. Grebenyuk ${ }^{122}$, Z.D. Greenwood ${ }^{78, m}$, K. Gregersen ${ }^{77}$, I.M. Gregor ${ }^{42}$, P. Grenier ${ }^{144}$, J. Griffiths ${ }^{8}$, A.A. Grillo ${ }^{138}$, K. Grimm ${ }^{71}$, S. Grinstein ${ }^{12, n}$, Ph. Gris ${ }^{34}$, Y.V. Grishkevich ${ }^{98}$, J.-F. Grivaz ${ }^{116}$, J.P. Grohs ${ }^{44}$, A. Grohsjean ${ }^{42}$, E. Gross ${ }^{173}$, J. Grosse-Knetter ${ }^{54}$, G.C. Grossi ${ }^{134 a, 134 b}$, J. Groth-Jensen ${ }^{173}$, Z.J. Grout ${ }^{150}$, L. Guan ${ }^{33 b}$, F. Guescini ${ }^{49}$, D. Guest ${ }^{177}$, O. Gueta ${ }^{154}$, C. Guicheney ${ }^{34}$, E. Guido ${ }^{50 a, 50 b}$, T. Guillemin ${ }^{116}$, S. Guindon ${ }^{2}$, U. Gul ${ }^{53}$, C. Gumpert ${ }^{44}$, J. Gunther ${ }^{127}$, J. Guo ${ }^{35}$, S. Gupta ${ }^{119}$, P. Gutierrez ${ }^{112}$, N.G. Gutierrez Ortiz ${ }^{53}$, C. Gutschow ${ }^{77}$, N. Guttman ${ }^{154}$, C. Guyot ${ }^{137}$, C. Gwenlan ${ }^{119}$, C.B. Gwilliam ${ }^{73}$, A. Haas ${ }^{109}$, C. Haber ${ }^{15}$, H.K. Hadavand ${ }^{8}$, N. Haddad ${ }^{136 e}$, P. Haefner ${ }^{21}$, S. Hageböck ${ }^{21}$, Z. Hajduk ${ }^{39}$, H. Hakobyan ${ }^{178}$, M. Haleem ${ }^{42}$, D. Hall ${ }^{119}$, G. Halladjian ${ }^{89}$, K. Hamacher ${ }^{176}$, P. Hamal ${ }^{114}$, K. Hamano ${ }^{170}$, M. Hamer ${ }^{54}$, A. Hamilton ${ }^{146 a}$, S. Hamilton ${ }^{162}$, P.G. Hamnett ${ }^{42}$, L. Han ${ }^{33 b}$, K. Hanagaki ${ }^{117}$, K. Hanawa ${ }^{156}$, M. Hance ${ }^{15}$, P. Hanke ${ }^{58 a}$, R. Hanna ${ }^{137}$, J.B. Hansen ${ }^{36}$,

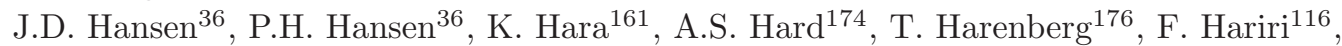
S. Harkusha ${ }^{91}$, D. Harper ${ }^{88}$, R.D. Harrington ${ }^{46}$, O.M. Harris ${ }^{139}$, P.F. Harrison ${ }^{171}$, F. Hartjes ${ }^{106}$, S. Hasegawa ${ }^{102}$, Y. Hasegawa ${ }^{141}$, A. Hasib ${ }^{112}$, S. Hassani ${ }^{137}$, S. Haug ${ }^{17}$, M. Hauschild $^{30}$, R. Hauser ${ }^{89}$, M. Havranek ${ }^{126}$, C.M. Hawkes ${ }^{18}$, R.J. Hawkings ${ }^{30}$, A.D. Hawkins ${ }^{80}$, T. Hayashi $^{161}$, D. Hayden ${ }^{89}$, C.P. Hays ${ }^{119}$, H.S. Hayward ${ }^{73}$, S.J. Haywood ${ }^{130}$, S.J. Head ${ }^{18}$, T. Heck ${ }^{82}$, V. Hedberg ${ }^{80}$, L. Heelan ${ }^{8}$, S. Heim ${ }^{121}$, T. Heim ${ }^{176}$, B. Heinemann ${ }^{15}$, L. Heinrich ${ }^{109}$, J. Hejbal ${ }^{126}$, L. Helary ${ }^{22}$, C. Heller ${ }^{99}$, M. Heller ${ }^{30}$, S. Hellman 147a,147b, D. Hellmich ${ }^{21}$, C. Helsens ${ }^{30}$, J. Henderson ${ }^{119}$, R.C.W. Henderson ${ }^{71}$, Y. Heng ${ }^{174}$, C. Hengler ${ }^{42}$, A. Henrichs ${ }^{177}$, A.M. Henriques Correia ${ }^{30}$, S. Henrot-Versille ${ }^{116}$, C. Hensel ${ }^{54}$, G.H. Herbert ${ }^{16}$, Y. Hernández Jiménez ${ }^{168}$, R. Herrberg-Schubert ${ }^{16}$, G. Herten ${ }^{48}$, R. Hertenberger ${ }^{99}$, L. Hervas ${ }^{30}$, 
G.G. Hesketh ${ }^{77}$, N.P. Hessey ${ }^{106}$, R. Hickling ${ }^{75}$, E. Higón-Rodriguez ${ }^{168}$, E. Hill ${ }^{170}$, J.C. Hill ${ }^{28}$, K.H. Hiller ${ }^{42}$, S. Hillert ${ }^{21}$, S.J. Hillier ${ }^{18}$, I. Hinchliffe ${ }^{15}$, E. Hines ${ }^{121}$, M. Hirose ${ }^{158}$, D. Hirschbuehl ${ }^{176}$, J. Hobbs ${ }^{149}$, N. Hod ${ }^{106}$, M.C. Hodgkinson ${ }^{140}$, P. Hodgson ${ }^{140}$, A. Hoecker ${ }^{30}$, M.R. Hoeferkamp ${ }^{104}$, J. Hoffman ${ }^{40}$, D. Hoffmann ${ }^{84}$, J.I. Hofmann ${ }^{58 a}$, M. Hohlfeld ${ }^{82}$, T.R. Holmes ${ }^{15}$, T.M. Hong ${ }^{121}$, L. Hooft van Huysduynen ${ }^{109}$, J-Y. Hostachy ${ }^{55}$, S. Hou ${ }^{152}$,

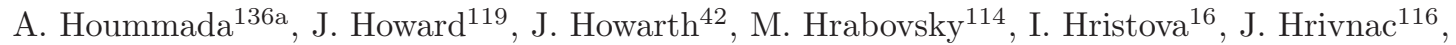
T. Hryn'ova ${ }^{5}$, C. Hsu ${ }^{146 c}$, P.J. Hsu ${ }^{82}$, S.-C. Hsu ${ }^{139}$, D. Hu ${ }^{35}$, X. Hu ${ }^{25}$, Y. Huang ${ }^{42}$, Z. Hubacek ${ }^{30}$, F. Hubaut ${ }^{84}$, F. Huegging ${ }^{21}$, T.B. Huffman ${ }^{119}$, E.W. Hughes ${ }^{35}$, G. Hughes ${ }^{71}$, M. Huhtinen ${ }^{30}$,

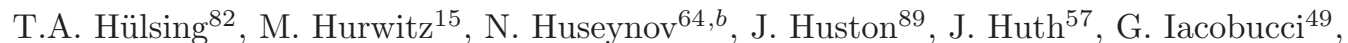
G. Iakovidis ${ }^{10}$, I. Ibragimov ${ }^{142}$, L. Iconomidou-Fayard ${ }^{116}$, E. Ideal ${ }^{177}$, P. Iengo ${ }^{103 a}$, O. Igonkina ${ }^{106}$, T. Iizawa ${ }^{172}$, Y. Ikegami ${ }^{65}$, K. Ikematsu ${ }^{142}$, M. Ikeno ${ }^{65}$, Y. Ilchenko ${ }^{31, o}$, D. Iliadis ${ }^{155}$, N. Ilic ${ }^{159}$,

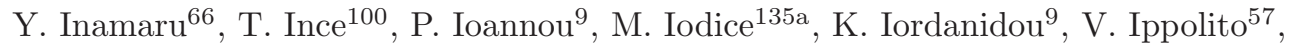
A. Irles Quiles ${ }^{168}$, C. Isaksson ${ }^{167}$, M. Ishino ${ }^{67}$, M. Ishitsuka ${ }^{158}$, R. Ishmukhametov ${ }^{110}$, C. Issever ${ }^{119}$, S. Istin ${ }^{19 a}$, J.M. Iturbe Ponce ${ }^{83}$, R. Iuppa ${ }^{134 a, 134 b}$, J. Ivarsson ${ }^{80}$, W. Iwanski ${ }^{39}$, H. Iwasaki ${ }^{65}$, J.M. Izen ${ }^{41}$, V. Izzo ${ }^{103 a}$, B. Jackson ${ }^{121}$, M. Jackson ${ }^{73}$, P. Jackson ${ }^{1}$, M.R. Jaekel ${ }^{30}$, V. Jain ${ }^{2}$, K. Jakobs ${ }^{48}$, S. Jakobsen ${ }^{30}$, T. Jakoubek ${ }^{126}$, J. Jakubek ${ }^{127}$, D.O. Jamin ${ }^{152}$, D.K. Jana ${ }^{78}$, E. Jansen ${ }^{77}$, H. Jansen ${ }^{30}$, J. Janssen ${ }^{21}$, M. Janus ${ }^{171}$, G. Jarlskog ${ }^{80}$, N. Javadov ${ }^{64, b}$, T. Javůrek ${ }^{48}$, L. Jeanty ${ }^{15}$, J. Jejelava ${ }^{51 a, p}$, G.-Y. Jeng ${ }^{151}$, D. Jennens ${ }^{87}$, P. Jenni ${ }^{48, q}$, J. Jentzsch ${ }^{43}$, C. Jeske ${ }^{171}$, S. Jézéquel ${ }^{5}$, H. Ji ${ }^{174}$, W. Ji ${ }^{82}$, J. Jia ${ }^{149}$, Y. Jiang ${ }^{33 b}$, M. Jimenez Belenguer ${ }^{42}$, S. Jin ${ }^{33 a}$, A. Jinaru ${ }^{26 a}$, O. Jinnouchi ${ }^{158}$, M.D. Joergensen ${ }^{36}$, K.E. Johansson ${ }^{147 a, 147 b}$, P. Johansson ${ }^{140}$, K.A. Johns ${ }^{7}$, K. Jon-And ${ }^{147 a, 147 b}$, G. Jones ${ }^{171}$, R.W.L. Jones ${ }^{71}$, T.J. Jones ${ }^{73}$, J. Jongmanns ${ }^{58 a}$, P.M. Jorge ${ }^{125 a, 125 b}$, K.D. Joshi ${ }^{83}$, J. Jovicevic ${ }^{148}$, X. Ju ${ }^{174}$, C.A. Jung ${ }^{43}$, R.M. Jungst ${ }^{30}$, P. Jussel ${ }^{61}$, A. Juste Rozas ${ }^{12, n}$, M. Kaci ${ }^{168}$, A. Kaczmarska ${ }^{39}$, M. Kado ${ }^{116}$, H. Kagan ${ }^{110}$, M. Kagan ${ }^{144}$, E. Kajomovitz ${ }^{45}$, C.W. Kalderon ${ }^{119}$, S. Kama ${ }^{40}$, A. Kamenshchikov ${ }^{129}$, N. Kanaya ${ }^{156}$, M. Kaneda ${ }^{30}$, S. Kaneti ${ }^{28}$, V.A. Kantserov ${ }^{97}$, J. Kanzaki ${ }^{65}$, B. Kaplan ${ }^{109}$, A. Kapliy ${ }^{31}$, D. $\operatorname{Kar}^{53}$, K. Karakostas ${ }^{10}$, N. Karastathis ${ }^{10}$, M. Karnevskiy ${ }^{82}$, S.N. Karpov ${ }^{64}$, K. Karthik ${ }^{109}$, V. Kartvelishvili ${ }^{71}$, A.N. Karyukhin ${ }^{129}$, L. Kashif ${ }^{174}$, G. Kasieczka ${ }^{58 b}$, R.D. Kass ${ }^{110}$, A. Kastanas ${ }^{14}$, Y. Kataoka ${ }^{156}$, A. Katre ${ }^{49}$, J. Katzy ${ }^{42}$, V. Kaushik ${ }^{7}$, K. Kawagoe ${ }^{69}$, T. Kawamoto ${ }^{156}$, G. Kawamura ${ }^{54}$, S. Kazama ${ }^{156}$, V.F. Kazanin ${ }^{108}$, M.Y. Kazarinov ${ }^{64}$, R. Keeler ${ }^{170}$, R. Kehoe ${ }^{40}$, M. Keil ${ }^{54}$, J.S. Keller ${ }^{42}$, J.J. Kempster ${ }^{76}$, H. Keoshkerian ${ }^{5}$, O. Kepka ${ }^{126}$, B.P. Kerševan ${ }^{74}$, S. Kersten ${ }^{176}$, K. Kessoku ${ }^{156}$, J. Keung ${ }^{159}$, F. Khalil-zada ${ }^{11}$, H. Khandanyan ${ }^{147 a, 147 b}$, A. Khanov ${ }^{113}$, A. Khodinov ${ }^{97}$, A. Khomich ${ }^{58 a}$, T.J. Khoo ${ }^{28}$, G. Khoriauli ${ }^{21}$, A. Khoroshilov ${ }^{176}$, V. Khovanskiy ${ }^{96}$, E. Khramov ${ }^{64}$, J. Khubua ${ }^{51 b}$, H.Y. Kim ${ }^{8}$, H. Kim ${ }^{147 a, 147 b}$, S.H. Kim ${ }^{161}$, N. Kimura ${ }^{172}$, O. Kind ${ }^{16}$, B.T. King ${ }^{73}$, M. King ${ }^{168}$, R.S.B. King ${ }^{119}$, S.B. King ${ }^{169}$, J. Kirk ${ }^{130}$, A.E. Kiryunin ${ }^{100}$, T. Kishimoto ${ }^{66}$, D. Kisielewska ${ }^{38 a}$, F. Kiss ${ }^{48}$, T. Kittelmann ${ }^{124}$, K. Kiuchi ${ }^{161}$, E. Kladiva ${ }^{145 b}$, M. Klein ${ }^{73}$, U. Klein ${ }^{73}$, K. Kleinknecht ${ }^{82}$, P. Klimek ${ }^{147 a, 147 b}$, A. Klimentov ${ }^{25}$, R. Klingenberg ${ }^{43}$, J.A. Klinger ${ }^{83}$, T. Klioutchnikova ${ }^{30}$, P.F. Klok ${ }^{105}$, E.-E. Kluge ${ }^{58 a}$, P. Kluit ${ }^{106}$, S. Kluth ${ }^{100}$, E. Kneringer ${ }^{61}$, E.B.F.G. Knoops ${ }^{84}$, A. Knue ${ }^{53}$, D. Kobayashi ${ }^{158}$, T. Kobayashi ${ }^{156}$, M. Kobel ${ }^{44}$, M. Kocian ${ }^{144}$, P. Kodys ${ }^{128}$, P. Koevesarki ${ }^{21}$, T. Koffas ${ }^{29}$, E. Koffeman ${ }^{106}$, L.A. Kogan ${ }^{119}$, S. Kohlmann ${ }^{176}$, Z. Kohout ${ }^{127}$, T. Kohriki ${ }^{65}$, T. Koi ${ }^{144}$, H. Kolanoski ${ }^{16}$, I. Koletsou ${ }^{5}$, J. Koll ${ }^{89}$, A.A. Komar ${ }^{95, *}$, Y. Komori ${ }^{156}$, T. Kondo ${ }^{65}$, N. Kondrashova ${ }^{42}$, K. Köneke ${ }^{48}$, A.C. König ${ }^{105}$, S. König ${ }^{82}$, T. Kono ${ }^{65, r}$, R. Konoplich ${ }^{109, s}$, N. Konstantinidis ${ }^{77}$, R. Kopeliansky ${ }^{153}$, S. Koperny ${ }^{38 a}$, L. Köpke ${ }^{82}$,

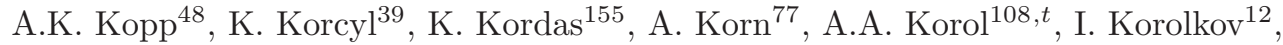
E.V. Korolkova ${ }^{140}$, V.A. Korotkov ${ }^{129}$, O. Kortner ${ }^{100}$, S. Kortner ${ }^{100}$, V.V. Kostyukhin ${ }^{21}$, V.M. Kotov ${ }^{64}$, A. Kotwal ${ }^{45}$, C. Kourkoumelis ${ }^{9}$, V. Kouskoura ${ }^{155}$, A. Koutsman ${ }^{160 a}$, R. Kowalewski ${ }^{170}$, T.Z. Kowalski ${ }^{38 a}$, W. Kozanecki ${ }^{137}$, A.S. Kozhin ${ }^{129}$, V. Kral ${ }^{127}$, V.A. Kramarenko ${ }^{98}$, G. Kramberger ${ }^{74}$, D. Krasnopevtsev ${ }^{97}$, M.W. Krasny ${ }^{79}$, A. Krasznahorkay ${ }^{30}$, 


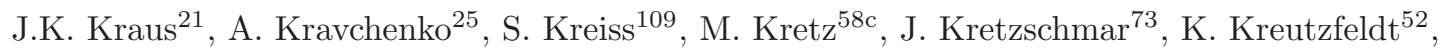
P. Krieger ${ }^{159}$, K. Kroeninger ${ }^{54}$, H. Kroha ${ }^{100}$, J. Kroll ${ }^{121}$, J. Kroseberg ${ }^{21}$, J. Krstic ${ }^{13 a}$, U. Kruchonak ${ }^{64}$, H. Krüger ${ }^{21}$, T. Kruker ${ }^{17}$, N. Krumnack ${ }^{63}$, Z.V. Krumshteyn ${ }^{64}$, A. Kruse ${ }^{174}$, M.C. Kruse ${ }^{45}$, M. Kruskal ${ }^{22}$, T. Kubota ${ }^{87}$, S. Kuday ${ }^{4 a}$, S. Kuehn ${ }^{48}$, A. Kugel ${ }^{58 c}$, A. Kuhl ${ }^{138}$, T. Kuhl ${ }^{42}$, V. Kukhtin ${ }^{64}$, Y. Kulchitsky ${ }^{91}$, S. Kuleshov ${ }^{32 b}$, M. Kuna ${ }^{133 a, 133 b}$, J. Kunkle ${ }^{121}$, A. Kupco ${ }^{126}$, H. Kurashige ${ }^{66}$, Y.A. Kurochkin ${ }^{91}$, R. Kurumida ${ }^{66}$, V. Kus ${ }^{126}$, E.S. Kuwertz ${ }^{148}$, M. Kuze ${ }^{158}$, J. Kvita ${ }^{114}$, A. La Rosa ${ }^{49}$, L. La Rotonda ${ }^{37 a, 37 b}$, C. Lacasta ${ }^{168}$, F. Lacava ${ }^{133 a, 133 b}$,

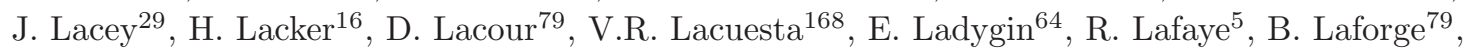
T. Lagouri ${ }^{177}$, S. Lai ${ }^{48}$, H. Laier ${ }^{58 a}$, L. Lambourne ${ }^{77}$, S. Lammers ${ }^{60}$, C.L. Lampen ${ }^{7}$, W. Lampl ${ }^{7}$, E. Lançon ${ }^{137}$, U. Landgraf ${ }^{48}$, M.P.J. Landon ${ }^{75}$, V.S. Lang ${ }^{58 a}$, A.J. Lankford ${ }^{164}$, F. Lanni ${ }^{25}$, K. Lantzsch ${ }^{30}$, S. Laplace ${ }^{79}$, C. Lapoire ${ }^{21}$, J.F. Laporte ${ }^{137}$, T. Lari ${ }^{90 a}$, M. Lassnig ${ }^{30}$, P. Laurelli ${ }^{47}$, W. Lavrijsen ${ }^{15}$, A.T. Law ${ }^{138}$, P. Laycock ${ }^{73}$, B.T. Le ${ }^{55}$, O. Le Dortz ${ }^{79}$, E. Le Guirriec ${ }^{84}$, E. Le Menedeu ${ }^{12}$, T. LeCompte ${ }^{6}$, F. Ledroit-Guillon ${ }^{55}$, C.A. Lee ${ }^{152}$, H. Lee ${ }^{106}$, J.S.H. Lee ${ }^{117}$,

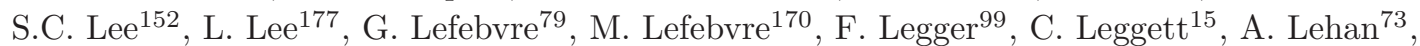
M. Lehmacher ${ }^{21}$, G. Lehmann Miotto ${ }^{30}$, X. Lei ${ }^{7}$, W.A. Leight ${ }^{29}$, A. Leisos ${ }^{155}$, A.G. Leister ${ }^{177}$, M.A.L. Leite ${ }^{24 d}$, R. Leitner ${ }^{128}$, D. Lellouch ${ }^{173}$, B. Lemmer ${ }^{54}$, K.J.C. Leney ${ }^{77}$, T. Lenz ${ }^{106}$, G. Lenzen ${ }^{176}$, B. Lenzi ${ }^{30}$, R. Leone ${ }^{7}$, S. Leone ${ }^{123 a, 123 b}$, K. Leonhardt ${ }^{44}$, C. Leonidopoulos ${ }^{46}$, S. Leontsinis ${ }^{10}$, C. Leroy ${ }^{94}$, C.G. Lester ${ }^{28}$, C.M. Lester ${ }^{121}$, M. Levchenko ${ }^{122}$, J. Levêque ${ }^{5}$, D. Levin ${ }^{88}$, L.J. Levinson ${ }^{173}$, M. Levy ${ }^{18}$, A. Lewis ${ }^{119}$, G.H. Lewis ${ }^{109}$, A.M. Leyko ${ }^{21}$, M. Leyton ${ }^{41}$,

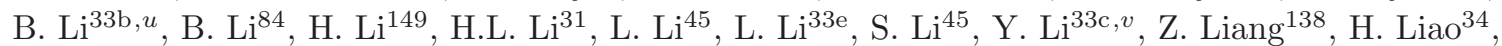

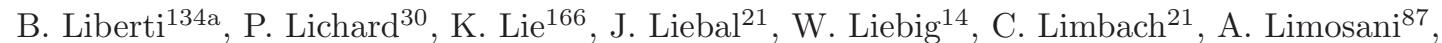
S.C. $\operatorname{Lin}^{152, w}$, T.H. $\operatorname{Lin}^{82}$, F. Linde ${ }^{106}$, B.E. Lindquist ${ }^{149}$, J.T. Linnemann ${ }^{89}$, E. Lipeles ${ }^{121}$, A. Lipniacka ${ }^{14}$, M. Lisovyi ${ }^{42}$, T.M. Liss ${ }^{166}$, D. Lissauer ${ }^{25}$, A. Lister ${ }^{169}$, A.M. Litke ${ }^{138}$, B. Liu ${ }^{152}$,

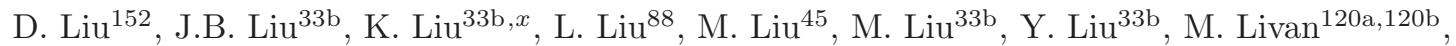
S.S.A. Livermore ${ }^{119}$, A. Lleres ${ }^{55}$, J. Llorente Merino ${ }^{81}$, S.L. Lloyd ${ }^{75}$, F. Lo Sterzo ${ }^{152}$, E. Lobodzinska ${ }^{42}$, P. Loch ${ }^{7}$, W.S. Lockman ${ }^{138}$, T. Loddenkoetter ${ }^{21}$, F.K. Loebinger ${ }^{83}$, A.E. Loevschall-Jensen ${ }^{36}$, A. Loginov ${ }^{177}$, C.W. Loh ${ }^{169}$, T. Lohse ${ }^{16}$, K. Lohwasser ${ }^{42}$, M. Lokajicek ${ }^{126}$, V.P. Lombardo ${ }^{5}$, B.A. Long ${ }^{22}$, J.D. Long ${ }^{88}$, R.E. Long ${ }^{71}$, L. Lopes ${ }^{125 a}$, D. Lopez Mateos ${ }^{57}$, B. Lopez Paredes ${ }^{140}$, I. Lopez Paz ${ }^{12}$, J. Lorenz ${ }^{99}$, N. Lorenzo Martinez ${ }^{60}$,

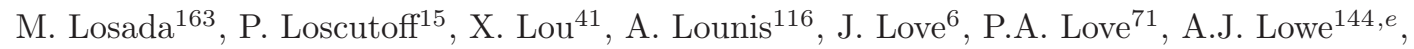
F. Lu ${ }^{33 a}$, H.J. Lubatti ${ }^{139}$, C. Luci ${ }^{133 a, 133 b}$, A. Lucotte ${ }^{55}$, F. Luehring ${ }^{60}$, W. Lukas ${ }^{61}$, L. Luminari ${ }^{133 a}$, O. Lundberg ${ }^{147 a, 147 b}$, B. Lund-Jensen ${ }^{148}$, M. Lungwitz ${ }^{82}$, D. Lynn ${ }^{25}$, R. Lysak $^{126}$, E. Lytken ${ }^{80}$, H. Ma ${ }^{25}$, L.L. Ma ${ }^{33 d}$, G. Maccarrone ${ }^{47}$, A. Macchiolo ${ }^{100}$, J. Machado Miguens ${ }^{125 a, 125 b}$, D. Macina ${ }^{30}$, D. Madaffari ${ }^{84}$, R. Madar ${ }^{48}$, H.J. Maddocks ${ }^{71}$, W.F. Mader ${ }^{44}$, A. Madsen ${ }^{167}$, M. Maeno ${ }^{8}$, T. Maeno ${ }^{25}$, E. Magradze ${ }^{54}$, K. Mahboubi ${ }^{48}$, J. Mahlstedt ${ }^{106}$, S. Mahmoud ${ }^{73}$, C. Maiani ${ }^{137}$, C. Maidantchik ${ }^{24 a}$, A.A. Maier ${ }^{100}$, A. Maio ${ }^{125 a, 125 b, 125 d}$, S. Majewski ${ }^{115}$, Y. Makida ${ }^{65}$, N. Makovec ${ }^{116}$, P. Mal ${ }^{137, y}$, B. Malaescu ${ }^{79}$, Pa. Malecki ${ }^{39}$, V.P. Maleev ${ }^{122}$, F. Malek ${ }^{55}$, U. Mallik ${ }^{62}$, D. Malon ${ }^{6}$, C. Malone ${ }^{144}$, S. Maltezos ${ }^{10}$, V.M. Malyshev ${ }^{108}$, S. Malyukov ${ }^{30}$, J. Mamuzic ${ }^{13 b}$, B. Mandelli ${ }^{30}$, L. Mandelli ${ }^{90 a}$, I. Mandić ${ }^{74}$, R. Mandrysch ${ }^{62}$, J. Maneira ${ }^{125 a, 125 b}$, A. Manfredini ${ }^{100}$, L. Manhaes de Andrade Filho ${ }^{24 b}$, J.A. Manjarres Ramos ${ }^{160 b}$, A. Mann ${ }^{99}$, P.M. Manning ${ }^{138}$, A. Manousakis-Katsikakis ${ }^{9}$, B. Mansoulie ${ }^{137}$, R. Mantifel ${ }^{86}$, L. Mapelli ${ }^{30}$, L. March ${ }^{168}$, J.F. Marchand ${ }^{29}$, G. Marchiori ${ }^{79}$, M. Marcisovsky ${ }^{126}$, C.P. Marino ${ }^{170}$, M. Marjanovic ${ }^{13 a}$, C.N. Marques ${ }^{125 a}$, F. Marroquim ${ }^{24 a}$, S.P. Marsden ${ }^{83}$, Z. Marshall ${ }^{15}$, L.F. Marti ${ }^{17}$, S. Marti-Garcia ${ }^{168}$, B. Martin ${ }^{30}$, B. Martin ${ }^{89}$, T.A. Martin ${ }^{171}$, V.J. Martin ${ }^{46}$, B. Martin dit Latour ${ }^{14}$, H. Martinez ${ }^{137}$, M. Martinez ${ }^{12, n}$, S. Martin-Haugh ${ }^{130}$, A.C. Martyniuk ${ }^{77}$, M. Marx ${ }^{139}$, F. Marzano ${ }^{133 a}$, A. Marzin ${ }^{30}$, L. Masetti ${ }^{82}$, T. Mashimo ${ }^{156}$, R. Mashinistov ${ }^{95}$, J. Masik ${ }^{83}$, A.L. Maslennikov ${ }^{108}$, I. Massa ${ }^{20 a, 20 b}$, N. Massol ${ }^{5}$, P. Mastrandrea ${ }^{149}$, A. Mastroberardino ${ }^{37 a, 37 b}$, T. Masubuchi ${ }^{156}$, P. Mättig ${ }^{176}$, J. Mattmann ${ }^{82}$, 
J. Maurer ${ }^{26 a}$, S.J. Maxfield ${ }^{73}$, D.A. Maximov ${ }^{108, t}$, R. Mazini ${ }^{152}$, L. Mazzaferro134a,134b, G. Mc Goldrick ${ }^{159}$, S.P. Mc Kee ${ }^{88}$, A. $\mathrm{McCarn}^{88}$, R.L. McCarthy ${ }^{149}$, T.G. McCarthy ${ }^{29}$, N.A. McCubbin ${ }^{130}$, K.W. McFarlane ${ }^{56, *}$, J.A. Mcfayden ${ }^{77}$, G. Mchedlidze ${ }^{54}$, S.J. McMahon ${ }^{130}$, R.A. McPherson ${ }^{170, i}$, A. Meade ${ }^{85}$, J. Mechnich ${ }^{106}$, M. Medinnis ${ }^{42}$, S. Meehan ${ }^{31}$, S. Mehlhase ${ }^{99}$, A. Mehta ${ }^{73}$, K. Meier ${ }^{58 a}$, C. Meineck ${ }^{99}$, B. Meirose ${ }^{80}$, C. Melachrinos ${ }^{31}$, B.R. Mellado Garcia ${ }^{146 c}$, F. Meloni ${ }^{17}$, A. Mengarelli ${ }^{20 a, 20 b}$, S. Menke ${ }^{100}$, E. Meoni ${ }^{162}$, K.M. Mercurio ${ }^{57}$, S. Mergelmeyer ${ }^{21}$, N. Meric ${ }^{137}$, P. Mermod ${ }^{49}$, L. Merola ${ }^{103 a, 103 b}$, C. Meroni ${ }^{90 a}$, F.S. Merritt ${ }^{31}$, H. Merritt ${ }^{110}$, A. Messina ${ }^{30, z}$, J. Metcalfe ${ }^{25}$, A.S. Mete ${ }^{164}$, C. Meyer ${ }^{82}$, C. Meyer ${ }^{31}$, J-P. Meyer ${ }^{137}$, J. Meyer ${ }^{30}$, R.P. Middleton ${ }^{130}$, S. Migas ${ }^{73}$, L. Mijović ${ }^{21}$, G. Mikenberg ${ }^{173}$, M. Mikestikova ${ }^{126}$, M. Mikuž ${ }^{74}$, D.W. Miller ${ }^{31}$, C. Mills ${ }^{46}$, A. Milov ${ }^{173}$, D.A. Milstead ${ }^{147 a, 147 b}$, D. Milstein ${ }^{173}$, A.A. Minaenko ${ }^{129}$, I.A. Minashvili ${ }^{64}$, A.I. Mincer ${ }^{109}$, B. Mindur ${ }^{38 a}$, M. Mineev ${ }^{64}$, Y. Ming ${ }^{174}$, L.M. Mir ${ }^{12}$, G. Mirabelli ${ }^{133 a}$, T. Mitani ${ }^{172}$, J. Mitrevski ${ }^{99}$, V.A. Mitsou ${ }^{168}$, S. Mitsui ${ }^{65}$, A. Miucci ${ }^{49}$, P.S. Miyagawa ${ }^{140}$, J.U. Mjörnmark ${ }^{80}$, T. Moa ${ }^{147 a, 147 b}$, K. Mochizuki ${ }^{84}$, S. Mohapatra ${ }^{35}$, W. Mohr ${ }^{48}$, S. Molanderr47a,147b, R. Moles-Valls ${ }^{168}$, K. Mönig ${ }^{42}$, C. Monini ${ }^{55}$, J. Monk ${ }^{36}$, E. Monnier ${ }^{84}$, J. Montejo Berlingen ${ }^{12}$, F. Monticelli ${ }^{70}$, S. Monzani133a,133b, R.W. Moore ${ }^{3}$,

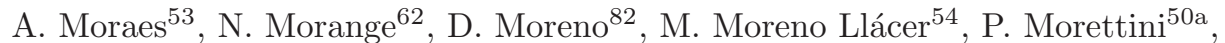
M. Morgenstern ${ }^{44}$, M. Morii ${ }^{57}$, S. Moritz ${ }^{82}$, A.K. Morley ${ }^{148}$, G. Mornacchi ${ }^{30}$, J.D. Morris ${ }^{75}$, L. Morvaj ${ }^{102}$, H.G. Moser ${ }^{100}$, M. Mosidze ${ }^{51 b}$, J. Moss ${ }^{110}$, K. Motohashi ${ }^{158}$, R. Mount ${ }^{144}$, E. Mountricha ${ }^{25}$, S.V. Mouraviev ${ }^{95, *}$, E.J.W. Moyse ${ }^{85}$, S. Muanza ${ }^{84}$, R.D. Mudd ${ }^{18}$, F. Mueller ${ }^{58 a}$, J. Mueller ${ }^{124}$, K. Mueller ${ }^{21}$, T. Mueller ${ }^{28}$, T. Mueller ${ }^{82}$, D. Muenstermann ${ }^{49}$, Y. Munwes ${ }^{154}$, J.A. Murillo Quijada ${ }^{18}$, W.J. Murray ${ }^{171,130}$, H. Musheghyan ${ }^{54}$, E. Musto ${ }^{153}$, A.G. Myagkov ${ }^{129, a a}$, M. Myska ${ }^{127}$, O. Nackenhorst ${ }^{54}$, J. Nadal ${ }^{54}$, K. Nagai ${ }^{61}$, R. Nagai ${ }^{158}$, Y. Nagai ${ }^{84}$, K. Nagano ${ }^{65}$, A. Nagarkar ${ }^{110}$, Y. Nagasaka ${ }^{59}$, M. Nagel ${ }^{100}$, A.M. Nairz ${ }^{30}$, Y. Nakahama ${ }^{30}$, K. Nakamura ${ }^{65}$, T. Nakamura ${ }^{156}$, I. Nakano ${ }^{111}$, H. Namasivayam ${ }^{41}$, G. Nanava ${ }^{21}$, R. Narayan ${ }^{58 b}$,

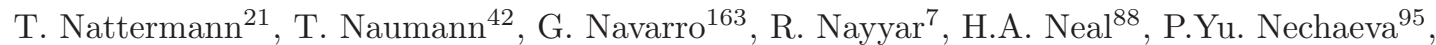
T.J. Neep ${ }^{83}$, A. Negri ${ }^{120 a, 120 b}$, G. Negri ${ }^{30}$, M. Negrini ${ }^{20 a}$, S. Nektarijevic ${ }^{49}$, A. Nelson ${ }^{164}$, T.K. Nelson ${ }^{144}$, S. Nemecek ${ }^{126}$, P. Nemethy ${ }^{109}$, A.A. Nepomuceno ${ }^{24 a}$, M. Nessi ${ }^{30, a b}$, M.S. Neubauer ${ }^{166}$, M. Neumann ${ }^{176}$, R.M. Neves ${ }^{109}$, P. Nevski ${ }^{25}$, P.R. Newman ${ }^{18}$, D.H. Nguyen ${ }^{6}$, R.B. Nickerson ${ }^{119}$, R. Nicolaidou ${ }^{137}$, B. Nicquevert ${ }^{30}$, J. Nielsen ${ }^{138}$, N. Nikiforou ${ }^{35}$, A. Nikiforov ${ }^{16}$, V. Nikolaenko ${ }^{129, a a}$, I. Nikolic-Audit ${ }^{79}$, K. Nikolics ${ }^{49}$, K. Nikolopoulos ${ }^{18}$, P. Nilsson ${ }^{8}$, Y. Ninomiya ${ }^{156}$, A. Nisati ${ }^{133 a}$, R. Nisius ${ }^{100}$, T. Nobe ${ }^{158}$, L. Nodulman ${ }^{6}$, M. Nomachi ${ }^{117}$, I. Nomidis ${ }^{155}$, S. Norberg ${ }^{112}$, M. Nordberg ${ }^{30}$, S. Nowak ${ }^{100}$, M. Nozaki ${ }^{65}$, L. Nozka ${ }^{114}$, K. Ntekas ${ }^{10}$, G. Nunes Hanninger ${ }^{87}$, T. Nunnemann ${ }^{99}$, E. Nurse ${ }^{77}$, F. Nuti ${ }^{87}$, B.J. O’Brien ${ }^{46}$, F. O'grady ${ }^{7}$, D.C. O’Neil ${ }^{143}$, V. O'Shea ${ }^{53}$, F.G. Oakham ${ }^{29, d}$, H. Oberlack ${ }^{100}$,

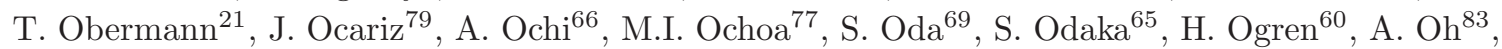

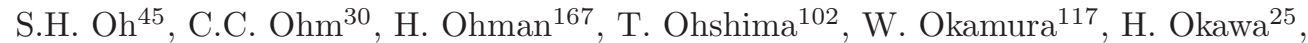
Y. Okumura ${ }^{31}$, T. Okuyama ${ }^{156}$, A. Olariu ${ }^{26 a}$, A.G. Olchevski ${ }^{64}$, S.A. Olivares Pino ${ }^{46}$, D. Oliveira Damazio ${ }^{25}$, E. Oliver Garcia ${ }^{168}$, A. Olszewski ${ }^{39}$, J. Olszowska ${ }^{39}$, A. Onofre ${ }^{125 a, 125 e}$, P.U.E. Onyisi ${ }^{31, o}$, C.J. Oram ${ }^{160 a}$, M.J. Oreglia ${ }^{31}$, Y. Oren ${ }^{154}$, D. Orestano ${ }^{135 a, 135 b}$,

N. Orlando $72 \mathrm{a}, 72 \mathrm{~b}$, C. Oropeza Barrera ${ }^{53}$, R.S. Orr ${ }^{159}$, B. Osculati ${ }^{50 a, 50 b}$, R. Ospanov ${ }^{121}$, G. Otero y Garzon ${ }^{27}$, H. Otono ${ }^{69}$, M. Ouchrif ${ }^{136 d}$, E.A. Ouellette ${ }^{170}$, F. Ould-Saada ${ }^{118}$,

A. Ouraou ${ }^{137}$, K.P. Oussoren ${ }^{106}$, Q. Ouyang ${ }^{33 a}$, A. Ovcharova ${ }^{15}$, M. Owen $^{83}$, V.E. Ozcan ${ }^{19 a}$, N. Ozturk ${ }^{8}$, K. Pachal ${ }^{119}$, A. Pacheco Pages ${ }^{12}$, C. Padilla Aranda ${ }^{12}$, M. Pagáčová ${ }^{48}$, S. Pagan Griso ${ }^{15}$, E. Paganis ${ }^{140}$, C. Pahl ${ }^{100}$, F. Paige ${ }^{25}$, P. Pais ${ }^{85}$, K. Pajchel ${ }^{118}$, G. Palacino ${ }^{160 b}$, S. Palestini ${ }^{30}$, M. Palka ${ }^{38 b}$, D. Pallin ${ }^{34}$, A. Palma ${ }^{125 a, 125 b}$, J.D. Palmer ${ }^{18}$, Y.B. Pan ${ }^{174}$,

E. Panagiotopoulou ${ }^{10}$, J.G. Panduro Vazquez ${ }^{76}$, P. Pani ${ }^{106}$, N. Panikashvili ${ }^{88}$, S. Panitkin ${ }^{25}$, D. Pantea ${ }^{26 a}$, L. Paolozzi ${ }^{134 a, 134 b}$, Th.D. Papadopoulou ${ }^{10}$, K. Papageorgiou ${ }^{155, l}$, A. Paramonov ${ }^{6}$, D. Paredes Hernandez ${ }^{34}$, M.A. Parker ${ }^{28}$, F. Parodi ${ }^{50 a, 50 b}$, J.A. Parsons ${ }^{35}$, U. Parzefall ${ }^{48}$, 
E. Pasqualucci133a, S. Passaggio ${ }^{50 a}$, A. Passeri1 ${ }^{135 a}$, F. Pastore ${ }^{135 a, 135 b, *}$, Fr. Pastore ${ }^{76}$, G. Pásztor ${ }^{29}$, S. Pataraia ${ }^{176}$, N.D. Patel $^{151}$, J.R. Pater ${ }^{83}$, S. Patricelli103a,103b ${ }^{10}$ T. Pauly ${ }^{30}$, J. Pearce ${ }^{170}$, M. Pedersen ${ }^{118}$, S. Pedraza Lopez ${ }^{168}$, R. Pedro ${ }^{125 a, 125 b}$, S.V. Peleganchuk ${ }^{108}$, D. Pelikan ${ }^{167}$, H. Peng ${ }^{33 b}$, B. Penning ${ }^{31}$, J. Penwell ${ }^{60}$, D.V. Perepelitsa ${ }^{25}$, E. Perez Codina ${ }^{160 a}$, M.T. Pérez García-Estañ ${ }^{168}$, V. Perez Reale ${ }^{35}$, L. Perini ${ }^{90 a}, 90 b, H$. Pernegger $^{30}$, R. Perrino ${ }^{72 a}$, R. Peschke ${ }^{42}$, V.D. Peshekhonov ${ }^{64}$, K. Peters ${ }^{30}$, R.F.Y. Peters ${ }^{83}$, B.A. Petersen ${ }^{30}$, T.C. Petersen ${ }^{36}$, E. Petit ${ }^{42}$, A. Petridis ${ }^{147 a, 147 b}$, C. Petridou ${ }^{155}$, E. Petrolo ${ }^{133 a}$, F. Petrucci ${ }^{135 a, 135 b}$, N.E. Pettersson ${ }^{158}$, R. Pezoa ${ }^{32 b}$, P.W. Phillips ${ }^{130}$, G. Piacquadio ${ }^{144}$, E. Pianori ${ }^{171}$, A. Picazio ${ }^{49}$, E. Piccaro ${ }^{75}$, M. Piccinini ${ }^{20 a, 20 b}$, R. Piegaia ${ }^{27}$, D.T. Pignotti ${ }^{110}$, J.E. Pilcher ${ }^{31}$, A.D. Pilkington ${ }^{77}$, J. Pina125a,125b,125d, M. Pinamonti165a,165c,ac, A. Pinder ${ }^{119}$, J.L. Pinfold ${ }^{3}$, A. Pingel ${ }^{36}$, B. Pinto ${ }^{125 a}$, S. Pires ${ }^{79}$, M. Pitt ${ }^{173}$, C. Pizio 90a,90b L. Plazak $^{145 a}$, M.-A. Pleier ${ }^{25}$, V. Pleskot ${ }^{128}$, E. Plotnikova ${ }^{64}$, P. Plucinski147a,147b, S. Poddar ${ }^{58 a}$, F. Podlyski $^{34}$, R. Poettgen ${ }^{82}$, L. Poggioli ${ }^{116}$, D. Pohl ${ }^{21}$, M. Pohl ${ }^{49}$, G. Polesello ${ }^{120 a}$, A. Policicchio ${ }^{37 a, 37 b}$, R. Polifka ${ }^{159}$, A. Polini ${ }^{20 a}$, C.S. Pollard ${ }^{45}$, V. Polychronakos ${ }^{25}$, K. Pommès ${ }^{30}$, L. Pontecorvo ${ }^{133 a}$, B.G. Pope ${ }^{89}$, G.A. Popeneciu ${ }^{26 b}$, D.S. Popovic ${ }^{13 a}$, A. Poppleton ${ }^{30}$, X. Portell Bueso ${ }^{12}$, S. Pospisil ${ }^{127}$, K. Potamianos ${ }^{15}$, I.N. Potrap ${ }^{64}$, C.J. Potter ${ }^{150}$, C.T. Potter ${ }^{115}$, G. Poulard ${ }^{30}$,

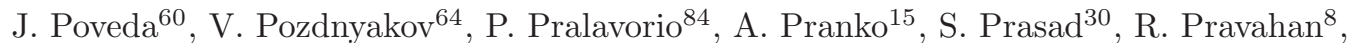
S. Prell ${ }^{63}$, D. Price ${ }^{83}$, J. Price ${ }^{73}$, L.E. Price ${ }^{6}$, D. Prieur ${ }^{124}$, M. Primavera ${ }^{72 a}$, M. Proissl ${ }^{46}$, K. Prokofiev ${ }^{47}$, F. Prokoshin ${ }^{32 b}$, E. Protopapadaki ${ }^{137}$, S. Protopopescu ${ }^{25}$, J. Proudfoot ${ }^{6}$, M. Przybycien ${ }^{38 a}$, H. Przysiezniak ${ }^{5}$, E. Ptacek ${ }^{115}$, D. Puddu ${ }^{135 a, 135 b}$, E. Pueschel ${ }^{85}$, D. Puldon ${ }^{149}$,

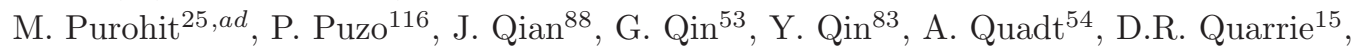
W.B. Quayle ${ }^{165 a, 165 b}$, M. Queitsch-Maitland ${ }^{83}$, D. Quilty ${ }^{53}$, A. Qureshi ${ }^{160 b}$, V. Radeka ${ }^{25}$, V. Radescu ${ }^{42}$, S.K. Radhakrishnan ${ }^{149}$, P. Radloff ${ }^{115}$, P. Rados ${ }^{87}$, F. Ragusa ${ }^{90 a, 90 b}$, G. Rahal ${ }^{179}$, S. Rajagopalan ${ }^{25}$, M. Rammensee ${ }^{30}$, A.S. Randle-Conde ${ }^{40}$, C. Rangel-Smith ${ }^{167}$, K. Rao ${ }^{164}$, F. Rauscher ${ }^{99}$, T.C. Rave ${ }^{48}$, T. Ravenscroft ${ }^{53}$, M. Raymond ${ }^{30}$, A.L. $\operatorname{Read}^{118}$, N.P. Readioff ${ }^{73}$, D.M. Rebuzzi ${ }^{120 a, 120 b}$, A. Redelbach ${ }^{175}$, G. Redlinger ${ }^{25}$, R. Reece ${ }^{138}$, K. Reeves ${ }^{41}$, L. Rehnisch ${ }^{16}$, H. Reisin ${ }^{27}$, M. Relich ${ }^{164}$, C. Rembser ${ }^{30}$, H. Ren ${ }^{33 a}$, Z.L. Ren ${ }^{152}$, A. Renaud ${ }^{116}$, M. Rescigno ${ }^{133 a}$,

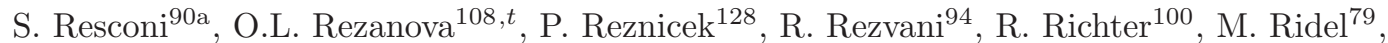
P. Rieck ${ }^{16}$, J. Rieger ${ }^{54}$, M. Rijssenbeek ${ }^{149}$, A. Rimoldi ${ }^{120 a, 120 b}$, L. Rinaldi ${ }^{20 a}$, E. Ritsch $^{61}$, I. Riu ${ }^{12}$, F. Rizatdinova ${ }^{113}$, E. Rizvi ${ }^{75}$, S.H. Robertson ${ }^{86, i}$, A. Robichaud-Veronneau ${ }^{86}$, D. Robinson ${ }^{28}$, J.E.M. Robinson ${ }^{83}$, A. Robson ${ }^{53}$, C. Roda ${ }^{123 a, 123 b}$, L. Rodrigues ${ }^{30}$, S. Roe $^{30}$, O. Røhne ${ }^{118}$, S. Rolli ${ }^{162}$, A. Romaniouk ${ }^{97}$, M. Romano ${ }^{20 a}, 20 b$, E. Romero Adam ${ }^{168}$, N. Rompotis ${ }^{139}$, L. Roos ${ }^{79}$, E. Ros ${ }^{168}$, S. Rosati ${ }^{133 a}$, K. Rosbach ${ }^{49}$, M. Rose $^{76}$, P.L. Rosendahl ${ }^{14}$, O. Rosenthal ${ }^{142}$, V. Rossetti 147a,147b, E. Rossi ${ }^{103 a, 103 b}$, L.P. Rossi ${ }^{50 a}$, R. Rosten ${ }^{139}$, M. Rotaru ${ }^{26 a}$, I. Roth ${ }^{173}$, J. Rothberg ${ }^{139}$, D. Rousseau ${ }^{116}$, C.R. Royon ${ }^{137}$, A. Rozanov ${ }^{84}$, Y. Rozen ${ }^{153}$, X. Ruan ${ }^{146 c}$, F. Rubbo ${ }^{12}$, I. Rubinskiy ${ }^{42}$, V.I. Rud ${ }^{98}$, C. Rudolph ${ }^{44}$, M.S. Rudolph ${ }^{159}$, F. Rühr ${ }^{48}$, A. Ruiz-Martinez ${ }^{30}$, Z. Rurikova ${ }^{48}$, N.A. Rusakovich ${ }^{64}$, A. Ruschke ${ }^{99}$, J.P. Rutherfoord ${ }^{7}$, N. Ruthmann ${ }^{48}$, Y.F. Ryabov ${ }^{122}$, M. Rybar ${ }^{128}$, G. Rybkin ${ }^{116}$, N.C. Ryder ${ }^{119}$, A.F. Saavedra ${ }^{151}$, S. Sacerdoti ${ }^{27}$, A. Saddique ${ }^{3}$, I. Sadeh ${ }^{154}$, H.F-W. Sadrozinski ${ }^{138}$, R. Sadykov ${ }^{64}$, F. Safai Tehrani ${ }^{133 a}$, H. Sakamoto ${ }^{156}$, Y. Sakurai ${ }^{172}$, G. Salamanna135a,135b ${ }^{13 .}$ Salamon ${ }^{134 a}$, M. Saleem ${ }^{112}$, D. Salek ${ }^{106}$, P.H. Sales De Bruin ${ }^{139}$, D. Salihagic ${ }^{100}$, A. Salnikov ${ }^{144}$, J. Salt ${ }^{168}$, B.M. Salvachua Ferrando ${ }^{6}$, D. Salvatore ${ }^{37 a, 37 b}$, F. Salvatore ${ }^{150}$, A. Salvucci ${ }^{105}$, A. Salzburger ${ }^{30}$, D. Sampsonidis ${ }^{155}$, A. Sanchez ${ }^{103 a, 103 b}$, J. Sánchez ${ }^{168}$, V. Sanchez Martinez ${ }^{168}$, H. Sandaker ${ }^{14}$, R.L. Sandbach ${ }^{75}$, H.G. Sander ${ }^{82}$, M.P. Sanders ${ }^{99}$, M. Sandhoff ${ }^{176}$, T. Sandoval ${ }^{28}$, C. Sandoval ${ }^{163}$, R. Sandstroem ${ }^{100}$, D.P.C. Sankey ${ }^{130}$, A. Sansoni ${ }^{47}$, C. Santoni ${ }^{34}$, R. Santonico ${ }^{134 a, 134 b}$, H. Santos ${ }^{125 a}$, I. Santoyo Castillo ${ }^{150}$, K. Sapp ${ }^{124}$, A. Sapronov ${ }^{64}$, J.G. Saraiva ${ }^{125 a, 125 d}$, B. Sarrazin ${ }^{21}$, G. Sartisohn ${ }^{176}$, O. Sasaki ${ }^{65}$, Y. Sasaki ${ }^{156}$, G. Sauvage ${ }^{5, *}$, E. Sauvan ${ }^{5}$, P. Savard ${ }^{159, d}$, D.O. Savu ${ }^{30}$, C. Sawyer ${ }^{119}$, L. Sawyer ${ }^{78, m}$, D.H. Saxon ${ }^{53}$, J. Saxon ${ }^{121}$, 
C. Sbarra ${ }^{20 a}$, A. Sbrizzi ${ }^{3}$, T. Scanlon 77 , D.A. Scannicchio ${ }^{164}$, M. Scarcella ${ }^{151}$, V. Scarfone ${ }^{37 a, 37 b}$, J. Schaarschmidt ${ }^{173}$, P. Schacht ${ }^{100}$, D. Schaefer ${ }^{121}$, R. Schaefer ${ }^{42}$, S. Schaepe ${ }^{21}$, S. Schaetzel ${ }^{58 b}$, U. Schäfer ${ }^{82}$, A.C. Schaffer ${ }^{116}$, D. Schaile ${ }^{99}$, R.D. Schamberger ${ }^{149}$, V. Scharf ${ }^{58 a}$,

V.A. Schegelsky ${ }^{122}$, D. Scheirich ${ }^{128}$, M. Schernau ${ }^{164}$, M.I. Scherzer ${ }^{35}$, C. Schiavi ${ }^{50 a, 50 b,}$ J. Schieck ${ }^{99}$, C. Schillo ${ }^{48}$, M. Schioppa ${ }^{37 a, 37 b}$, S. Schlenker ${ }^{30}$, E. Schmidt ${ }^{48}$, K. Schmieden ${ }^{30}$, C. Schmitt ${ }^{82}$, C. Schmitt ${ }^{99}$, S. Schmitt ${ }^{58 b}$, B. Schneider ${ }^{17}$, Y.J. Schnellbach ${ }^{73}$, U. Schnoor ${ }^{44}$, L. Schoeffel ${ }^{137}$, A. Schoening ${ }^{58 b}$, B.D. Schoenrock ${ }^{89}$, A.L.S. Schorlemmer ${ }^{54}$, M. Schott ${ }^{82}$, D. Schouten ${ }^{160 a}$, J. Schovancova ${ }^{25}$, S. Schramm ${ }^{159}$, M. Schreyer ${ }^{175}$, C. Schroeder ${ }^{82}$, N. Schuh ${ }^{82}$, M.J. Schultens ${ }^{21}$, H.-C. Schultz-Coulon ${ }^{58 a}$, H. Schulz ${ }^{16}$, M. Schumacher ${ }^{48}$, B.A. Schumm ${ }^{138}$, Ph. Schune ${ }^{137}$, C. Schwanenberger ${ }^{83}$, A. Schwartzman ${ }^{144}$, Ph. Schwegler ${ }^{100}$, Ph. Schwemling ${ }^{137}$, R. Schwienhorst ${ }^{89}$, J. Schwindling ${ }^{137}$, T. Schwindt ${ }^{21}$, M. Schwoerer ${ }^{5}$, F.G. Sciacca ${ }^{17}$, E. Scifo ${ }^{116}$, G. Sciolla ${ }^{23}$, W.G. Scott ${ }^{130}$, F. Scuri ${ }^{123 a, 123 b}$, F. Scutti ${ }^{21}$, J. Searcy ${ }^{88}$, G. Sedov ${ }^{42}$, E. Sedykh ${ }^{122}$, S.C. Seidel ${ }^{104}$, A. Seiden ${ }^{138}$, F. Seifert ${ }^{127}$, J.M. Seixas ${ }^{24 a}$, G. Sekhniaidze ${ }^{103 a}$, S.J. Sekula ${ }^{40}$, K.E. Selbach ${ }^{46}$, D.M. Seliverstov ${ }^{122, *}$, G. Sellers ${ }^{73}$, N. Semprini-Cesari ${ }^{20 a, 20 b}$, C. Serfon ${ }^{30}$, L. Serin ${ }^{116}$, L. Serkin ${ }^{54}$, T. Serre ${ }^{84}$, R. Seuster ${ }^{160 a}$, H. Severini ${ }^{112}$, T. Sfiligoj ${ }^{74}$, F. Sforza ${ }^{100}$, A. Sfyrla ${ }^{30}$, E. Shabalina ${ }^{54}$, M. Shamim ${ }^{115}$, L.Y. Shan ${ }^{33 a}$, R. Shang ${ }^{166}$, J.T. Shank ${ }^{22}$, M. Shapiro ${ }^{15}$, P.B. Shatalov ${ }^{96}$, K. Shaw ${ }^{165 a, 165 b}$, C.Y. Shehu ${ }^{150}$, P. Sherwood ${ }^{77}$, L. Shi ${ }^{152, a e}$, S. Shimizu ${ }^{66}$, C.O. Shimmin ${ }^{164}$, M. Shimojima ${ }^{101}$, M. Shiyakova ${ }^{64}$, A. Shmeleva ${ }^{95}$, M.J. Shochet ${ }^{31}$, D. Short ${ }^{119}$, S. Shrestha ${ }^{63}$, E. Shulga ${ }^{97}$, M.A. Shupe ${ }^{7}$, S. Shushkevich ${ }^{42}$, P. Sicho ${ }^{126}$, O. Sidiropoulou ${ }^{155}$, D. Sidorov ${ }^{113}$, A. Sidoti133a ${ }^{133}$ F. Siegert ${ }^{44}$, Dj. Sijacki ${ }^{13 a}$, J. Silva ${ }^{125 a, 125 d}$, Y. Silver ${ }^{154}$, D. Silverstein ${ }^{144}$, S.B. Silverstein ${ }^{147 a}$, V. Simak ${ }^{127}$, O. Simard ${ }^{5}$, Lj. Simic ${ }^{13 a}$, S. Simion ${ }^{116}$, E. Simioni ${ }^{82}$, B. Simmons ${ }^{77}$, R. Simoniello90a,90b, M. Simonyan ${ }^{36}$, P. Sinervo ${ }^{159}$, N.B. Sinev ${ }^{115}$, V. Sipica ${ }^{142}$, G. Siragusa ${ }^{175}$, A. Sircar ${ }^{78}$, A.N. Sisakyan ${ }^{64, *}$, S.Yu. Sivoklokov ${ }^{98}$, J. Sjölin ${ }^{147 a, 147 b}$, T.B. Sjursen ${ }^{14}$, H.P. Skottowe ${ }^{57}$, K.Yu. Skovpen ${ }^{108}$, P. Skubic ${ }^{112}$, M. Slater ${ }^{18}$, T. Slavicek ${ }^{127}$, K. Sliwa ${ }^{162}$, V. Smakhtin ${ }^{173}$, B.H. Smart ${ }^{46}$, L. Smestad ${ }^{14}$, S.Yu. Smirnov ${ }^{97}$, Y. Smirnov ${ }^{97}$, L.N. Smirnova ${ }^{98, a f}$, O. Smirnova ${ }^{80}$, K.M. Smith ${ }^{53}$, M. Smizanska ${ }^{71}$, K. Smolek ${ }^{127}$, A.A. Snesarev ${ }^{95}$, G. Snidero ${ }^{75}$, S. Snyder ${ }^{25}$, R. Sobie ${ }^{170, i}$,

F. Socher ${ }^{44}$, A. Soffer ${ }^{154}$, D.A. Soh ${ }^{152, a e}$, C.A. Solans ${ }^{30}$, M. Solar ${ }^{127}$, J. Solc ${ }^{127}$, E.Yu. Soldatov ${ }^{97}$, U. Soldevila ${ }^{168}$, E. Solfaroli Camillocci ${ }^{133 a, 133 b}$, A.A. Solodkov ${ }^{129}$, A. Soloshenko ${ }^{64}$, O.V. Solovyanov ${ }^{129}$, V. Solovyev ${ }^{122}$, P. Sommer ${ }^{48}$, H.Y. Song ${ }^{33 b}$, N. Soni ${ }^{1}$, A. Sood ${ }^{15}$, A. Sopczak ${ }^{127}$, B. Sopko ${ }^{127}$, V. Sopko ${ }^{127}$, V. Sorin ${ }^{12}$, M. Sosebee ${ }^{8}$, R. Soualah ${ }^{165 a, 165 c}$, P. Soueid ${ }^{94}$, A.M. Soukharev ${ }^{108}$, D. South ${ }^{42}$, S. Spagnolo ${ }^{72 a, 72 b}$, F. Spanò ${ }^{76}$, W.R. Spearman ${ }^{57}$, R. Spighi ${ }^{20 a}$, G. Spigo ${ }^{30}$, M. Spousta ${ }^{128}$, T. Spreitzer ${ }^{159}$, B. Spurlock ${ }^{8}$, R.D. St. Denis ${ }^{53, *}$, S. Staerz ${ }^{44}$, J. Stahlman ${ }^{121}$, R. Stamen ${ }^{58 a}$, E. Stanecka ${ }^{39}$, R.W. Stanek ${ }^{6}$, C. Stanescu ${ }^{135 a}$, M. Stanescu-Bellu ${ }^{42}$, M.M. Stanitzki ${ }^{42}$, S. Stapnes ${ }^{118}$, E.A. Starchenko ${ }^{129}$, J. Stark ${ }^{55}$, P. Staroba ${ }^{126}$, P. Starovoitov ${ }^{42}$, R. Staszewski ${ }^{39}$, P. Stavina ${ }^{145 a, *}$, P. Steinberg ${ }^{25}$, B. Stelzer ${ }^{143}$, H.J. Stelzer ${ }^{30}$, O. Stelzer-Chilton ${ }^{160 a}$, H. Stenzel ${ }^{52}$, S. Stern ${ }^{100}$, G.A. Stewart ${ }^{53}$, J.A. Stillings ${ }^{21}$, M.C. Stockton ${ }^{86}$, M. Stoebe ${ }^{86}$, G. Stoicea ${ }^{26 a}$, P. Stolte ${ }^{54}$, S. Stonjek ${ }^{100}$, A.R. Stradling ${ }^{8}$, A. Straessner ${ }^{44}$, M.E. Stramaglia ${ }^{17}$, J. Strandberg ${ }^{148}$, S. Strandberg147a,147b, A. Strandlie ${ }^{118}$, E. Strauss ${ }^{144}$, M. Strauss ${ }^{112}$, P. Strizenec ${ }^{145 b}$, R. Ströhmer ${ }^{175}$, D.M. Strom ${ }^{115}$, R. Stroynowski ${ }^{40}$, S.A. Stucci ${ }^{17}$, B. Stugu ${ }^{14}$, N.A. Styles ${ }^{42}$, D. $\mathrm{Su}^{144}$, J. Su ${ }^{124}$, HS. Subramania ${ }^{3}$, R. Subramaniam ${ }^{78}$, A. Succurro ${ }^{12}$, Y. Sugaya ${ }^{117}$, C. Suhr ${ }^{107}$, M. Suk ${ }^{127}$, V.V. Sulin ${ }^{95}$, S. Sultansoy ${ }^{4 c}$, T. Sumida ${ }^{67}$, X. Sun ${ }^{33 a}$, J.E. Sundermann ${ }^{48}$, K. Suruliz ${ }^{140}$, G. Susinno ${ }^{37 a, 37 b}$, M.R. Sutton ${ }^{150}$, Y. Suzuki ${ }^{65}$, M. Svatos ${ }^{126}$, S. Swedish ${ }^{169}$, M. Swiatlowski ${ }^{144}$, I. Sykora ${ }^{145 a}$, T. Sykora ${ }^{128}$, D. Ta ${ }^{89}$, C. Taccini ${ }^{135 a, 135 b}$, K. Tackmann ${ }^{42}$, J. Taenzer ${ }^{159}$, A. Taffard ${ }^{164}$, R. Tafirout ${ }^{160 a}$, N. Taiblum ${ }^{154}$, Y. Takahashi ${ }^{102}$, H. Takai ${ }^{25}$, R. Takashima ${ }^{68}$, H. Takeda ${ }^{66}$, T. Takeshita ${ }^{141}$, Y. Takubo ${ }^{65}$, M. Talby ${ }^{84}$, A.A. Talyshev ${ }^{108, t}$, J.Y.C. Tam ${ }^{175}$, K.G. Tan ${ }^{87}$, J. Tanaka ${ }^{156}$, R. Tanaka ${ }^{116}$, S. Tanaka ${ }^{132}$, S. Tanaka ${ }^{65}$, A.J. Tanasijczuk ${ }^{143}$, B.B. Tannenwald ${ }^{110}$, N. Tannoury ${ }^{21}$, 
S. Tapprogge ${ }^{82}$, S. Tarem ${ }^{153}$, F. Tarrade ${ }^{29}$, G.F. Tartarelli ${ }^{90 a}$, P. Tas ${ }^{128}$, M. Tasevsky ${ }^{126}$, T. Tashiro ${ }^{67}$, E. Tassi ${ }^{37 a, 37 b}$, A. Tavares Delgado ${ }^{125 a, 125 b}$, Y. Tayalati136d , F.E. Taylor ${ }^{93}$, G.N. Taylor ${ }^{87}$, W. Taylor ${ }^{160 b}$, F.A. Teischinger ${ }^{30}$, M. Teixeira Dias Castanheira ${ }^{75}$, P. Teixeira-Dias ${ }^{76}$, K.K. Temming ${ }^{48}$, H. Ten Kate ${ }^{30}$, P.K. Teng ${ }^{152}$, J.J. Teoh ${ }^{117}$, S. Terada ${ }^{65}$, K. Terashi ${ }^{156}$, J. Terron ${ }^{81}$, S. Terzo ${ }^{100}$, M. Testa ${ }^{47}$, R.J. Teuscher ${ }^{159, i}$, J. Therhaag ${ }^{21}$, T. Theveneaux-Pelzer ${ }^{34}$, J.P. Thomas ${ }^{18}$, J. Thomas-Wilsker ${ }^{76}$, E.N. Thompson ${ }^{35}$, P.D. Thompson ${ }^{18}$, P.D. Thompson ${ }^{159}$, A.S. Thompson ${ }^{53}$, L.A. Thomsen ${ }^{36}$, E. Thomson ${ }^{121}$,

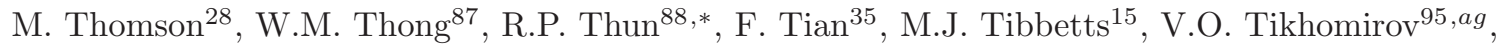

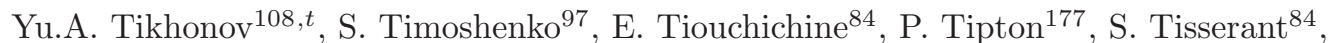
T. Todorov ${ }^{5}$, S. Todorova-Nova ${ }^{128}$, B. Toggerson ${ }^{7}$, J. Tojo ${ }^{69}$, S. Tokár ${ }^{145 a}$, K. Tokushuku ${ }^{65}$, K. Tollefson ${ }^{89}$, L. Tomlinson ${ }^{83}$, M. Tomoto ${ }^{102}$, L. Tompkins ${ }^{31}$, K. Toms ${ }^{104}$, N.D. Topilin ${ }^{64}$, E. Torrence ${ }^{115}$, H. Torres ${ }^{143}$, E. Torró Pastor ${ }^{168}$, J. Toth ${ }^{84, a h}$, F. Touchard ${ }^{84}$, D.R. Tovey ${ }^{140}$,

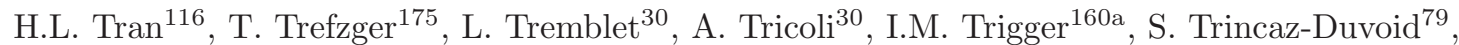
M.F. Tripiana ${ }^{12}$, N. Triplett ${ }^{25}$, W. Trischuk ${ }^{159}$, B. Trocmé ${ }^{55}$, C. Troncon ${ }^{90 a}$,

M. Trottier-McDonald ${ }^{143}$, M. Trovatelli ${ }^{135 a, 135 b}$, P. True ${ }^{89}$, M. Trzebinski ${ }^{39}$, A. Trzupek ${ }^{39}$, C. Tsarouchas ${ }^{30}$, J.C-L. Tseng ${ }^{119}$, P.V. Tsiareshka ${ }^{91}$, D. Tsionou ${ }^{137}$, G. Tsipolitis ${ }^{10}$,

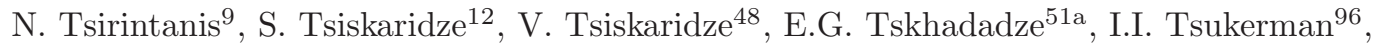

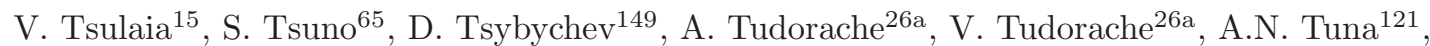
S.A. Tupputi ${ }^{20 a, 20 b}$, S. Turchikhin ${ }^{98, a f}$, D. Turecek ${ }^{127}$, I. Turk Cakir ${ }^{4 d}$, R. Turra ${ }^{90 a, 90 b,}$

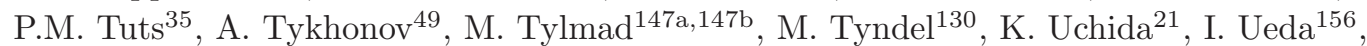

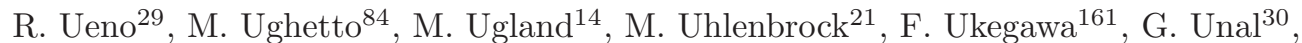

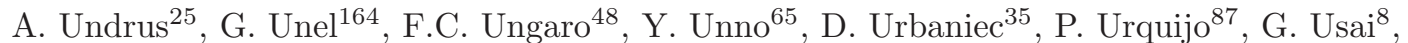

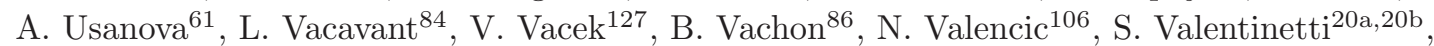
A. Valero ${ }^{168}$, L. Valery ${ }^{34}$, S. Valkar ${ }^{128}$, E. Valladolid Gallego ${ }^{168}$, S. Vallecorsa ${ }^{49}$,

J.A. Valls Ferrer ${ }^{168}$, W. Van Den Wollenberg ${ }^{106}$, P.C. Van Der Deij1 ${ }^{106}$, R. van der Geer ${ }^{106}$, H. van der Graaf ${ }^{106}$, R. Van Der Leeuw ${ }^{106}$, D. van der Ster $^{30}$, N. van Eldik ${ }^{30}$, P. van Gemmeren ${ }^{6}$, J. Van Nieuwkoop ${ }^{143}$, I. van Vulpen ${ }^{106}$, M.C. van $W_{0 e r d e n}{ }^{30}$, M. Vanadia ${ }^{133 a, 133 b}$, W. Vandelli ${ }^{30}$,

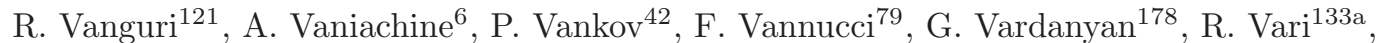

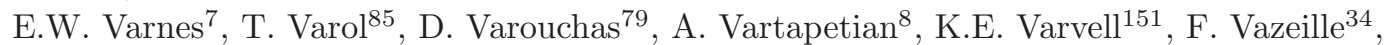
T. Vazquez Schroeder ${ }^{54}, J_{\text {. Veatch }}^{7}$, F. Veloso ${ }^{125 a, 125 c}$, S. Veneziano ${ }^{133 a}$, A. Ventura ${ }^{72 a, 72 b}$, D. Ventura ${ }^{85}$, M. Venturi ${ }^{170}$, N. Venturi ${ }^{159}$, A. Venturini ${ }^{23}$, V. Vercesi ${ }^{120 a}$, M. Verducci ${ }^{133 a, 133 b}$, W. Verkerke ${ }^{106}$, J.C. Vermeulen ${ }^{106}$, A. Vest ${ }^{44}$, M.C. Vetterli ${ }^{143, d}$, O. Viazlo ${ }^{80}$, I. Vichou ${ }^{166}$, T. Vickey ${ }^{146 c, a i}$, O.E. Vickey Boeriu ${ }^{146 c}$, G.H.A. Viehhauser ${ }^{119}$, S. Viel ${ }^{169}$, R. Vigne ${ }^{30}$,

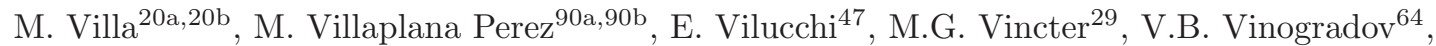

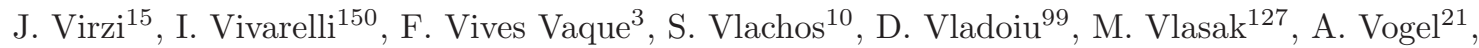
M. Vogel ${ }^{32 a}$, P. Vokac ${ }^{127}$, G. Volpi ${ }^{123 a, 123 b}$, M. Volpi ${ }^{87}$, H. von der $\operatorname{Schmitt}^{100}$,

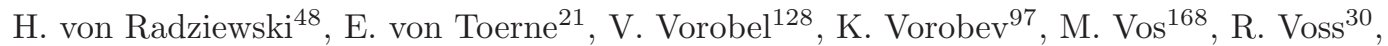
J.H. Vossebeld ${ }^{73}$, N. Vranjes ${ }^{137}$, M. Vranjes Milosavljevic ${ }^{106}$, V. Vrba ${ }^{126}$, M. Vreeswijk ${ }^{106}$, T. Vu Anh ${ }^{48}$, R. Vuillermet ${ }^{30}$, I. Vukotic ${ }^{31}$, Z. Vykydal ${ }^{127}$, P. Wagner ${ }^{21}$, W. Wagner ${ }^{176}$, H. Wahlberg ${ }^{70}$, S. Wahrmund ${ }^{44}$, J. Wakabayashi ${ }^{102}$, J. Walder ${ }^{71}$, R. Walker ${ }^{99}$, W. Walkowiak ${ }^{142}$,

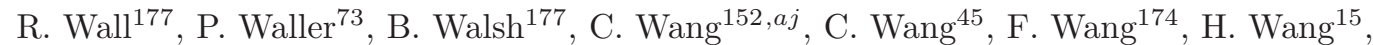
H. Wang ${ }^{40}$, J. Wang ${ }^{42}$, J. Wang ${ }^{33 a}$, K. Wang ${ }^{86}$, R. Wang ${ }^{104}$, S.M. Wang ${ }^{152}$, T. Wang ${ }^{21}$, X. Wang ${ }^{177}$, C. Wanotayaroj ${ }^{115}$, A. Warburton ${ }^{86}$, C.P. Ward ${ }^{28}$, D.R. Wardrope ${ }^{77}$, M. Warsinsky ${ }^{48}$, A. Washbrook ${ }^{46}$, C. Wasicki ${ }^{42}$, P.M. Watkins ${ }^{18}$, A.T. Watson ${ }^{18}$, I.J. Watson ${ }^{151}$, M.F. Watson ${ }^{18}$, G. Watts ${ }^{139}$, S. Watts ${ }^{83}$, B.M. Waugh ${ }^{77}$, S. Webb ${ }^{83}$, M.S. Weber ${ }^{17}$,

S.W. Weber ${ }^{175}$, J.S. Webster ${ }^{31}$, A.R. Weidberg ${ }^{119}$, P. Weigell ${ }^{100}$, B. Weinert ${ }^{60}$, J. Weingarten ${ }^{54}$, C. Weiser ${ }^{48}$, H. Weits ${ }^{106}$, P.S. Wells ${ }^{30}$, T. Wenaus ${ }^{25}$, D. Wendland ${ }^{16}$, Z. Weng ${ }^{152, a e}$, T. Wengler ${ }^{30}$, S. Wenig ${ }^{30}$, N. Wermes ${ }^{21}$, M. Werner ${ }^{48}$, P. Werner ${ }^{30}$, M. Wessels ${ }^{58 a}$, J. Wetter ${ }^{162}$, K. Whalen ${ }^{29}$, 


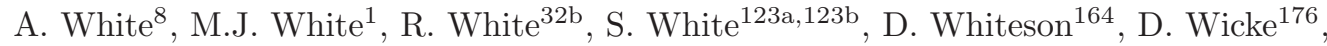
F.J. Wickens ${ }^{130}$, W. Wiedenmann ${ }^{174}$, M. Wielers ${ }^{130}$, P. Wienemann ${ }^{21}$, C. Wiglesworth ${ }^{36}$, L.A.M. Wiik-Fuchs ${ }^{21}$, P.A. Wijeratne ${ }^{77}$, A. Wildauer ${ }^{100}$, M.A. Wildt ${ }^{42, a k}$, H.G. Wilkens ${ }^{30}$, J.Z. Will ${ }^{99}$, H.H. Williams ${ }^{121}$, S. Williams ${ }^{28}$, C. Willis ${ }^{89}$, S. Willocq ${ }^{85}$, A. Wilson ${ }^{88}$, J.A. Wilson ${ }^{18}$, I. Wingerter-Seez ${ }^{5}$, F. Winklmeier ${ }^{115}$, B.T. Winter ${ }^{21}$, M. Wittgen ${ }^{144}$, T. Wittig ${ }^{43}$, J. Wittkowski ${ }^{99}$, S.J. Wollstadt ${ }^{82}$, M.W. Wolter ${ }^{39}$, H. Wolters ${ }^{125 a, 125 c}$, B.K. Wosiek ${ }^{39}$,

J. Wotschack ${ }^{30}$, M.J. Woudstra ${ }^{83}$, K.W. Wozniak ${ }^{39}$, M. Wright ${ }^{53}$, M. Wu ${ }^{55}$, S.L. Wu ${ }^{174}$, X. Wu ${ }^{49}$, Y. Wu ${ }^{88}$, E. Wulf ${ }^{35}$, T.R. Wyatt ${ }^{83}$, B.M. Wynne ${ }^{46}$, S. Xella ${ }^{36}$, M. Xiao ${ }^{137}$, D. Xu ${ }^{33 a}$, L. Xu $^{33 b, a l}$, B. Yabsley ${ }^{151}$, S. Yacoob ${ }^{146 b, a m}$, M. Yamada ${ }^{65}$, H. Yamaguchi ${ }^{156}$, Y. Yamaguchi ${ }^{156}$,

A. Yamamoto ${ }^{65}$, K. Yamamoto ${ }^{63}$, S. Yamamoto ${ }^{156}$, T. Yamamura ${ }^{156}$, T. Yamanaka ${ }^{156}$,

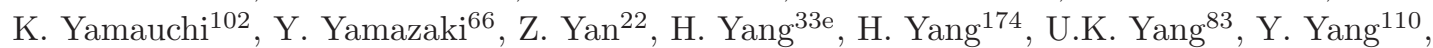

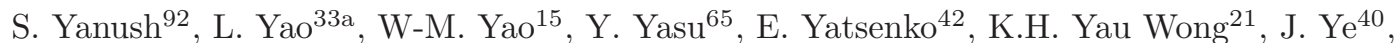
S. $\mathrm{Ye}^{25}$, A.L. Yen ${ }^{57}$, E. Yildirim ${ }^{42}$, M. Yilmaz ${ }^{4 b}$, R. Yoosoofmiya ${ }^{124}$, K. Yorita $^{172}$, R. Yoshida ${ }^{6}$, K. Yoshihara ${ }^{156}$, C. Young ${ }^{144}$, C.J.S. Young ${ }^{30}$, S. Youssef ${ }^{22}$, D.R. Yu ${ }^{15}$, J. Yu ${ }^{8}$, J.M. Yu ${ }^{88}$, J. Yu ${ }^{113}$, L. Yuan ${ }^{66}$, A. Yurkewicz ${ }^{107}$, I. Yusuff ${ }^{28, a n}$, B. Zabinski ${ }^{39}$, R. Zaidan ${ }^{62}$, A.M. Zaitsev ${ }^{129, a a}$, A. Zaman ${ }^{149}$, S. Zambito ${ }^{23}$, L. Zanello133a,133b, D. Zanzi ${ }^{100}$, C. Zeitnitz ${ }^{176}$, M. Zeman ${ }^{127}$, A. Zemla ${ }^{38 a}$, K. Zengel ${ }^{23}$, O. Zenin ${ }^{129}$, T. Ženiš ${ }^{145 a}$, D. Zerwas ${ }^{116}$,

G. Zevi della Porta ${ }^{57}$, D. Zhang ${ }^{88}$, F. Zhang ${ }^{174}$, H. Zhang ${ }^{89}$, J. Zhang ${ }^{6}$, L. Zhang ${ }^{152}$, X. Zhang ${ }^{33 d}$, Z. Zhang ${ }^{116}$, Z. Zhao ${ }^{33 b}$, A. Zhemchugov ${ }^{64}$, J. Zhong ${ }^{119}$, B. Zhou ${ }^{88}$, L. Zhou ${ }^{35}$, N. Zhou ${ }^{164}$, C.G. Zhu ${ }^{33 \mathrm{~d}}$, H. Zhu ${ }^{33 \mathrm{a}}$, J. Zhu $^{88}$, Y. Zhu ${ }^{33 \mathrm{~b}}, \mathrm{X}$. Zhuang $^{33 \mathrm{a}}$, K. Zhukov ${ }^{95}$, A. Zibell ${ }^{175}$, D. Zieminska ${ }^{60}$, N.I. Zimine ${ }^{64}$, C. Zimmermann ${ }^{82}$, R. Zimmermann ${ }^{21}$, S. Zimmermann ${ }^{21}$, S. Zimmermann ${ }^{48}$, Z. Zinonos ${ }^{54}$, M. Ziolkowski ${ }^{142}$, G. Zobernig ${ }^{174}$, A. Zoccoli ${ }^{20 a, 20 b}$, M. zur Nedden ${ }^{16}$, G. Zurzolo ${ }^{103 a, 103 b}$, V. Zutshi ${ }^{107}$, L. Zwalinski ${ }^{30}$.

1 Department of Physics, University of Adelaide, Adelaide, Australia

2 Physics Department, SUNY Albany, Albany NY, U.S.A.

3 Department of Physics, University of Alberta, Edmonton AB, Canada

4 (a) Department of Physics, Ankara University, Ankara; ${ }^{(b)}$ Department of Physics, Gazi University, Ankara; ${ }^{(c)}$ Division of Physics, TOBB University of Economics and Technology, Ankara; ${ }^{(d)}$ Turkish Atomic Energy Authority, Ankara, Turkey

5 LAPP, CNRS/IN2P3 and Université de Savoie, Annecy-le-Vieux, France

6 High Energy Physics Division, Argonne National Laboratory, Argonne IL, U.S.A.

7 Department of Physics, University of Arizona, Tucson AZ, U.S.A.

8 Department of Physics, The University of Texas at Arlington, Arlington TX, U.S.A.

9 Physics Department, University of Athens, Athens, Greece

10 Physics Department, National Technical University of Athens, Zografou, Greece

11 Institute of Physics, Azerbaijan Academy of Sciences, Baku, Azerbaijan

12 Institut de Física d'Altes Energies and Departament de Física de la Universitat Autònoma de Barcelona, Barcelona, Spain

13 (a) Institute of Physics, University of Belgrade, Belgrade; ${ }^{(b)}$ Vinca Institute of Nuclear Sciences, University of Belgrade, Belgrade, Serbia

14 Department for Physics and Technology, University of Bergen, Bergen, Norway

15 Physics Division, Lawrence Berkeley National Laboratory and University of California, Berkeley CA, U.S.A.

16 Department of Physics, Humboldt University, Berlin, Germany

17 Albert Einstein Center for Fundamental Physics and Laboratory for High Energy Physics, University of Bern, Bern, Switzerland

18 School of Physics and Astronomy, University of Birmingham, Birmingham, United Kingdom

19 (a) Department of Physics, Bogazici University, Istanbul; ${ }^{(b)}$ Department of Physics, Dogus University, Istanbul; ${ }^{(c)}$ Department of Physics Engineering, Gaziantep University, Gaziantep, Turkey 
20 (a) INFN Sezione di Bologna; ${ }^{(b)}$ Dipartimento di Fisica e Astronomia, Università di Bologna, Bologna, Italy

21 Physikalisches Institut, University of Bonn, Bonn, Germany

22 Department of Physics, Boston University, Boston MA, U.S.A.

23 Department of Physics, Brandeis University, Waltham MA, U.S.A.

24 (a) Universidade Federal do Rio De Janeiro COPPE/EE/IF, Rio de Janeiro; ${ }^{(b)}$ Federal University of Juiz de Fora (UFJF), Juiz de Fora; ${ }^{(c)}$ Federal University of Sao Joao del Rei (UFSJ), Sao Joao del Rei; ${ }^{(d)}$ Instituto de Fisica, Universidade de Sao Paulo, Sao Paulo, Brazil

25 Physics Department, Brookhaven National Laboratory, Upton NY, U.S.A.

26 (a) National Institute of Physics and Nuclear Engineering, Bucharest; ${ }^{(b)}$ National Institute for Research and Development of Isotopic and Molecular Technologies, Physics Department, Cluj Napoca; ${ }^{(c)}$ University Politehnica Bucharest, Bucharest; ${ }^{(d)}$ West University in Timisoara, Timisoara, Romania

27 Departamento de Física, Universidad de Buenos Aires, Buenos Aires, Argentina

28 Cavendish Laboratory, University of Cambridge, Cambridge, U.K.

29 Department of Physics, Carleton University, Ottawa ON, Canada

30 CERN, Geneva, Switzerland

31 Enrico Fermi Institute, University of Chicago, Chicago IL, U.S.A.

32 (a) Departamento de Física, Pontificia Universidad Católica de Chile, Santiago; ${ }^{(b)}$ Departamento de Física, Universidad Técnica Federico Santa María, Valparaíso, Chile

33 (a) Institute of High Energy Physics, Chinese Academy of Sciences, Beijing; ${ }^{(b)}$ Department of Modern Physics, University of Science and Technology of China, Anhui; ${ }^{(c)}$ Department of Physics, Nanjing University, Jiangsu; ${ }^{(d)}$ School of Physics, Shandong University, Shandong; ${ }^{(e)}$ Physics Department, Shanghai Jiao Tong University, Shanghai, China

34 Laboratoire de Physique Corpusculaire, Clermont Université and Université Blaise Pascal and CNRS/IN2P3, Clermont-Ferrand, France

35 Nevis Laboratory, Columbia University, Irvington NY, U.S.A.

36 Niels Bohr Institute, University of Copenhagen, Kobenhavn, Denmark

37 (a) INFN Gruppo Collegato di Cosenza, Laboratori Nazionali di Frascati; ${ }^{(b)}$ Dipartimento di Fisica, Università della Calabria, Rende, Italy

38 (a) AGH University of Science and Technology, Faculty of Physics and Applied Computer Science, Krakow; ${ }^{(b)}$ Marian Smoluchowski Institute of Physics, Jagiellonian University, Krakow, Poland

39 The Henryk Niewodniczanski Institute of Nuclear Physics, Polish Academy of Sciences, Krakow, Poland

40 Physics Department, Southern Methodist University, Dallas TX, U.S.A.

41 Physics Department, University of Texas at Dallas, Richardson TX, U.S.A.

42 DESY, Hamburg and Zeuthen, Germany

43 Institut für Experimentelle Physik IV, Technische Universität Dortmund, Dortmund, Germany

44 Institut für Kern- und Teilchenphysik, Technische Universität Dresden, Dresden, Germany

45 Department of Physics, Duke University, Durham NC, U.S.A.

46 SUPA - School of Physics and Astronomy, University of Edinburgh, Edinburgh, U.K.

47 INFN Laboratori Nazionali di Frascati, Frascati, Italy

48 Fakultät für Mathematik und Physik, Albert-Ludwigs-Universität, Freiburg, Germany

49 Section de Physique, Université de Genève, Geneva, Switzerland

50 (a) INFN Sezione di Genova; ${ }^{(b)}$ Dipartimento di Fisica, Università di Genova, Genova, Italy

51 (a) E. Andronikashvili Institute of Physics, Iv. Javakhishvili Tbilisi State University, Tbilisi; ${ }^{(b)}$ High Energy Physics Institute, Tbilisi State University, Tbilisi, Georgia

52 II Physikalisches Institut, Justus-Liebig-Universität Giessen, Giessen, Germany

53 SUPA - School of Physics and Astronomy, University of Glasgow, Glasgow, U.K.

54 II Physikalisches Institut, Georg-August-Universität, Göttingen, Germany

55 Laboratoire de Physique Subatomique et de Cosmologie, Université Grenoble-Alpes, CNRS/IN2P3, Grenoble, France 
57 Laboratory for Particle Physics and Cosmology, Harvard University, Cambridge MA, U.S.A.

58 (a) Kirchhoff-Institut für Physik, Ruprecht-Karls-Universität Heidelberg, Heidelberg; ${ }^{(b)}$ Physikalisches Institut, Ruprecht-Karls-Universität Heidelberg, Heidelberg; (c) ZITI Institut für technische Informatik, Ruprecht-Karls-Universität Heidelberg, Mannheim, Germany

59 Faculty of Applied Information Science, Hiroshima Institute of Technology, Hiroshima, Japan

60 Department of Physics, Indiana University, Bloomington IN, U.S.A.

61 Institut für Astro- und Teilchenphysik, Leopold-Franzens-Universität, Innsbruck, Austria

62 University of Iowa, Iowa City IA, U.S.A.

63 Department of Physics and Astronomy, Iowa State University, Ames IA, U.S.A.

64 Joint Institute for Nuclear Research, JINR Dubna, Dubna, Russia

65 KEK, High Energy Accelerator Research Organization, Tsukuba, Japan

66 Graduate School of Science, Kobe University, Kobe, Japan

67 Faculty of Science, Kyoto University, Kyoto, Japan

68 Kyoto University of Education, Kyoto, Japan

69 Department of Physics, Kyushu University, Fukuoka, Japan

70 Instituto de Física La Plata, Universidad Nacional de La Plata and CONICET, La Plata, Argentina

71 Physics Department, Lancaster University, Lancaster, U.K.

72 (a) INFN Sezione di Lecce; (b) Dipartimento di Matematica e Fisica, Università del Salento, Lecce, Italy

73 Oliver Lodge Laboratory, University of Liverpool, Liverpool, U.K.

74 Department of Physics, Jožef Stefan Institute and University of Ljubljana, Ljubljana, Slovenia

75 School of Physics and Astronomy, Queen Mary University of London, London, U.K.

76 Department of Physics, Royal Holloway University of London, Surrey, U.K.

77 Department of Physics and Astronomy, University College London, London, U.K.

78 Louisiana Tech University, Ruston LA, U.S.A.

79 Laboratoire de Physique Nucléaire et de Hautes Energies, UPMC and Université Paris-Diderot and CNRS/IN2P3, Paris, France

80 Fysiska institutionen, Lunds universitet, Lund, Sweden

81 Departamento de Fisica Teorica C-15, Universidad Autonoma de Madrid, Madrid, Spain

82 Institut für Physik, Universität Mainz, Mainz, Germany

83 School of Physics and Astronomy, University of Manchester, Manchester, U.K.

84 CPPM, Aix-Marseille Université and CNRS/IN2P3, Marseille, France

85 Department of Physics, University of Massachusetts, Amherst MA, U.S.A.

86 Department of Physics, McGill University, Montreal QC, Canada

87 School of Physics, University of Melbourne, Victoria, Australia

88 Department of Physics, The University of Michigan, Ann Arbor MI, U.S.A.

89 Department of Physics and Astronomy, Michigan State University, East Lansing MI, U.S.A.

90 (a) INFN Sezione di Milano; (b) Dipartimento di Fisica, Università di Milano, Milano, Italy

91 B.I. Stepanov Institute of Physics, National Academy of Sciences of Belarus, Minsk, Republic of Belarus

92 National Scientific and Educational Centre for Particle and High Energy Physics, Minsk, Republic of Belarus

93 Department of Physics, Massachusetts Institute of Technology, Cambridge MA, U.S.A.

94 Group of Particle Physics, University of Montreal, Montreal QC, Canada

95 P.N. Lebedev Institute of Physics, Academy of Sciences, Moscow, Russia

96 Institute for Theoretical and Experimental Physics (ITEP), Moscow, Russia

97 Moscow Engineering and Physics Institute (MEPhI), Moscow, Russia

98 D.V.Skobeltsyn Institute of Nuclear Physics, M.V.Lomonosov Moscow State University, Moscow, Russia

99 Fakultät für Physik, Ludwig-Maximilians-Universität München, München, Germany

100 Max-Planck-Institut für Physik (Werner-Heisenberg-Institut), München, Germany 
101 Nagasaki Institute of Applied Science, Nagasaki, Japan

102 Graduate School of Science and Kobayashi-Maskawa Institute, Nagoya University, Nagoya, Japan

103 (a) INFN Sezione di Napoli; ${ }^{(b)}$ Dipartimento di Fisica, Università di Napoli, Napoli, Italy

104 Department of Physics and Astronomy, University of New Mexico, Albuquerque NM, U.S.A.

105 Institute for Mathematics, Astrophysics and Particle Physics, Radboud University

Nijmegen/Nikhef, Nijmegen, Netherlands

106 Nikhef National Institute for Subatomic Physics and University of Amsterdam, Amsterdam, Netherlands

107 Department of Physics, Northern Illinois University, DeKalb IL, U.S.A.

108 Budker Institute of Nuclear Physics, SB RAS, Novosibirsk, Russia

109 Department of Physics, New York University, New York NY, U.S.A.

110 Ohio State University, Columbus OH, U.S.A.

111 Faculty of Science, Okayama University, Okayama, Japan

112 Homer L. Dodge Department of Physics and Astronomy, University of Oklahoma,

Norman OK, U.S.A.

113 Department of Physics, Oklahoma State University, Stillwater OK, U.S.A.

114 Palacký University, RCPTM, Olomouc, Czech Republic

115 Center for High Energy Physics, University of Oregon, Eugene OR, U.S.A.

116 LAL, Université Paris-Sud and CNRS/IN2P3, Orsay, France

117 Graduate School of Science, Osaka University, Osaka, Japan

118 Department of Physics, University of Oslo, Oslo, Norway

119 Department of Physics, Oxford University, Oxford, U.K.

120 (a) INFN Sezione di Pavia; ${ }^{(b)}$ Dipartimento di Fisica, Università di Pavia, Pavia, Italy

121 Department of Physics, University of Pennsylvania, Philadelphia PA, U.S.A.

122 Petersburg Nuclear Physics Institute, Gatchina, Russia

123 (a) INFN Sezione di Pisa; ${ }^{(b)}$ Dipartimento di Fisica E. Fermi, Università di Pisa, Pisa, Italy

124 Department of Physics and Astronomy, University of Pittsburgh, Pittsburgh PA, U.S.A.

125 (a) Laboratorio de Instrumentacao e Fisica Experimental de Particulas - LIP, Lisboa; ${ }^{(b)}$ Faculdade de Ciências, Universidade de Lisboa, Lisboa; ${ }^{(c)}$ Department of Physics, University of Coimbra, Coimbra; ${ }^{(d)}$ Centro de Física Nuclear da Universidade de Lisboa, Lisboa; ${ }^{(e)}$ Departamento de Fisica, Universidade do Minho, Braga; ${ }^{(f)}$ Departamento de Fisica Teorica y del Cosmos and CAFPE, Universidad de Granada, Granada (Spain); ${ }^{(g)}$ Dep Fisica and CEFITEC of Faculdade de Ciencias e Tecnologia, Universidade Nova de Lisboa, Caparica, Portugal

126 Institute of Physics, Academy of Sciences of the Czech Republic, Praha, Czech Republic

127 Czech Technical University in Prague, Praha, Czech Republic

128 Faculty of Mathematics and Physics, Charles University in Prague, Praha, Czech Republic

129 State Research Center Institute for High Energy Physics, Protvino, Russia

130 Particle Physics Department, Rutherford Appleton Laboratory, Didcot, U.K.

131 Physics Department, University of Regina, Regina SK, Canada

132 Ritsumeikan University, Kusatsu, Shiga, Japan

133 (a) INFN Sezione di Roma; ${ }^{(b)}$ Dipartimento di Fisica, Sapienza Università di Roma, Roma, Italy

134 (a) INFN Sezione di Roma Tor Vergata; ${ }^{(b)}$ Dipartimento di Fisica, Università di Roma Tor Vergata, Roma, Italy

135 (a) INFN Sezione di Roma Tre; ${ }^{(b)}$ Dipartimento di Matematica e Fisica, Università Roma Tre, Roma, Italy

136 (a) Faculté des Sciences Ain Chock, Réseau Universitaire de Physique des Hautes Energies Université Hassan II, Casablanca; ${ }^{(b)}$ Centre National de l'Energie des Sciences Techniques Nucleaires, Rabat; ${ }^{\left({ }^{c}\right)}$ Faculté des Sciences Semlalia, Université Cadi Ayyad, LPHEA-Marrakech; (d) Faculté des Sciences, Université Mohamed Premier and LPTPM, Oujda; ${ }^{(e)}$ Faculté des sciences, Université Mohammed V-Agdal, Rabat, Morocco

137 DSM/IRFU (Institut de Recherches sur les Lois Fondamentales de l'Univers), CEA Saclay (Commissariat à l'Energie Atomique et aux Energies Alternatives), Gif-sur-Yvette, France 
Santa Cruz CA, U.S.A.

139 Department of Physics, University of Washington, Seattle WA, U.S.A.

140 Department of Physics and Astronomy, University of Sheffield, Sheffield, U.K.

141 Department of Physics, Shinshu University, Nagano, Japan

142 Fachbereich Physik, Universität Siegen, Siegen, Germany

143 Department of Physics, Simon Fraser University, Burnaby BC, Canada

144 SLAC National Accelerator Laboratory, Stanford CA, U.S.A.

145 (a) Faculty of Mathematics, Physics $\& 3$ Informatics, Comenius University, Bratislava; ${ }^{(b)}$ Department of Subnuclear Physics, Institute of Experimental Physics of the Slovak Academy of Sciences, Kosice, Slovak Republic

146 (a) Department of Physics, University of Cape Town, Cape Town; ${ }^{\left({ }^{b}\right)}$ Department of Physics, University of Johannesburg, Johannesburg; ${ }^{(c)}$ School of Physics, University of the Witwatersrand, Johannesburg, South Africa

147 (a) Department of Physics, Stockholm University; ${ }^{(b)}$ The Oskar Klein Centre, Stockholm, Sweden

148 Physics Department, Royal Institute of Technology, Stockholm, Sweden

149 Departments of Physics \& Astronomy and Chemistry, Stony Brook University, Stony Brook NY, U.S.A.

150 Department of Physics and Astronomy, University of Sussex, Brighton, U.K.

151 School of Physics, University of Sydney, Sydney, Australia

152 Institute of Physics, Academia Sinica, Taipei, Taiwan

153 Department of Physics, Technion: Israel Institute of Technology, Haifa, Israel

154 Raymond and Beverly Sackler School of Physics and Astronomy, Tel Aviv University, Tel Aviv, Israel

155 Department of Physics, Aristotle University of Thessaloniki, Thessaloniki, Greece

156 International Center for Elementary Particle Physics and Department of Physics, The University of Tokyo, Tokyo, Japan

157 Graduate School of Science and Technology, Tokyo Metropolitan University, Tokyo, Japan

158 Department of Physics, Tokyo Institute of Technology, Tokyo, Japan

159 Department of Physics, University of Toronto, Toronto ON, Canada

160 (a) TRIUMF, Vancouver BC; ${ }^{(b)}$ Department of Physics and Astronomy, York University, Toronto ON, Canada

161 Faculty of Pure and Applied Sciences, University of Tsukuba, Tsukuba, Japan

162 Department of Physics and Astronomy, Tufts University, Medford MA, U.S.A.

163 Centro de Investigaciones, Universidad Antonio Narino, Bogota, Colombia

164 Department of Physics and Astronomy, University of California Irvine, Irvine CA, U.S.A.

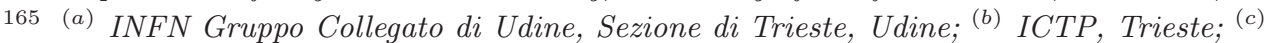

Dipartimento di Chimica, Fisica e Ambiente, Università di Udine, Udine, Italy

166 Department of Physics, University of Illinois, Urbana IL, U.S.A.

167 Department of Physics and Astronomy, University of Uppsala, Uppsala, Sweden

168 Instituto de Física Corpuscular (IFIC) and Departamento de Física Atómica, Molecular y Nuclear and Departamento de Ingeniería Electrónica and Instituto de Microelectrónica de Barcelona (IMB-CNM), University of Valencia and CSIC, Valencia, Spain

169 Department of Physics, University of British Columbia, Vancouver BC, Canada

170 Department of Physics and Astronomy, University of Victoria, Victoria BC, Canada

171 Department of Physics, University of Warwick, Coventry, U.K.

172 Waseda University, Tokyo, Japan

173 Department of Particle Physics, The Weizmann Institute of Science, Rehovot, Israel

174 Department of Physics, University of Wisconsin, Madison WI, U.S.A.

175 Fakultät für Physik und Astronomie, Julius-Maximilians-Universität, Würzburg, Germany

${ }_{176}$ Fachbereich C Physik, Bergische Universität Wuppertal, Wuppertal, Germany

177 Department of Physics, Yale University, New Haven CT, U.S.A. 
178 Yerevan Physics Institute, Yerevan, Armenia

179 Centre de Calcul de l'Institut National de Physique Nucléaire et de Physique des Particules (IN2P3), Villeurbanne, France

a Also at Department of Physics, King's College London, London, U.K.

${ }^{b}$ Also at Institute of Physics, Azerbaijan Academy of Sciences, Baku, Azerbaijan

c Also at Particle Physics Department, Rutherford Appleton Laboratory, Didcot, U.K.

${ }^{d}$ Also at TRIUMF, Vancouver BC, Canada

e Also at Department of Physics, California State University, Fresno CA, U.S.A.

${ }^{f}$ Also at Tomsk State University, Tomsk, Russia

$g$ Also at CPPM, Aix-Marseille Université and CNRS/IN2P3, Marseille, France

${ }^{h}$ Also at Università di Napoli Parthenope, Napoli, Italy

i Also at Institute of Particle Physics (IPP), Canada

j Also at Department of Physics, St. Petersburg State Polytechnical University, St. Petersburg, Russia

${ }^{k}$ Also at Chinese University of Hong Kong, China

${ }^{l}$ Also at Department of Financial and Management Engineering, University of the Aegean, Chios, Greece

$m$ Also at Louisiana Tech University, Ruston LA, U.S.A.

${ }^{n}$ Also at Institucio Catalana de Recerca i Estudis Avancats, ICREA, Barcelona, Spain

- Also at Department of Physics, The University of Texas at Austin, Austin TX, U.S.A.

${ }^{p}$ Also at Institute of Theoretical Physics, Ilia State University, Tbilisi, Georgia

$q$ Also at CERN, Geneva, Switzerland

${ }^{r}$ Also at Ochadai Academic Production, Ochanomizu University, Tokyo, Japan

$s$ Also at Manhattan College, New York NY, U.S.A.

${ }^{t}$ Also at Novosibirsk State University, Novosibirsk, Russia

${ }^{u}$ Also at Institute of Physics, Academia Sinica, Taipei, Taiwan

$v$ Also at LAL, Université Paris-Sud and CNRS/IN2P3, Orsay, France

w Also at Academia Sinica Grid Computing, Institute of Physics, Academia Sinica, Taipei, Taiwan

$x$ Also at Laboratoire de Physique Nucléaire et de Hautes Energies, UPMC and Université Paris-Diderot and CNRS/IN2P3, Paris, France

y Also at School of Physical Sciences, National Institute of Science Education and Research, Bhubaneswar, India

z Also at Dipartimento di Fisica, Sapienza Università di Roma, Roma, Italy

aa Also at Moscow Institute of Physics and Technology State University, Dolgoprudny, Russia

ab Also at Section de Physique, Université de Genève, Geneva, Switzerland

ac Also at International School for Advanced Studies (SISSA), Trieste, Italy

ad Also at Department of Physics and Astronomy, University of South Carolina, Columbia SC, U.S.A.

ae Also at School of Physics and Engineering, Sun Yat-sen University, Guangzhou, China

af Also at Faculty of Physics, M.V.Lomonosov Moscow State University, Moscow, Russia

ag Also at Moscow Engineering and Physics Institute (MEPhI), Moscow, Russia

ah Also at Institute for Particle and Nuclear Physics, Wigner Research Centre for Physics, Budapest, Hungary

ai Also at Department of Physics, Oxford University, Oxford, U.K.

aj Also at Department of Physics, Nanjing University, Jiangsu, China

ak Also at Institut für Experimentalphysik, Universität Hamburg, Hamburg, Germany

al Also at Department of Physics, The University of Michigan, Ann Arbor MI, U.S.A.

am Also at Discipline of Physics, University of KwaZulu-Natal, Durban, South Africa

an Also at University of Malaya, Department of Physics, Kuala Lumpur, Malaysia

* Deceased 